\title{
OPTIMAL FILTER PLACEMENT AND SIZING USING ANT COLONY OPTIMIZATION IN ELECTRICAL DISTRIBUTION SYSTEM
}

by

\section{Fawaz Masoud Alhaddad}

Submitted in partial fulfilment of the requirements for the degree of Master of Applied Science

at

Dalhousie University

Halifax, Nova Scotia

May 2014

(C) Copyright by Fawaz Masoud Alhaddad, 2014 


\section{Dedication}

I dedicate my thesis to my parents, my wife and my children Kinda and Mohammad 


\section{Table of Contents}

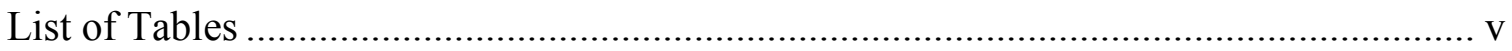

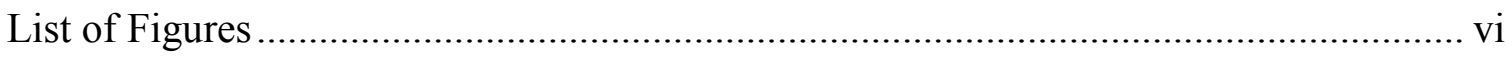

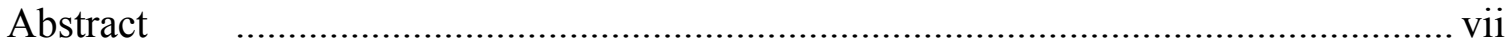

List of Abbreviations and Symbols Used ………………....................................... viii

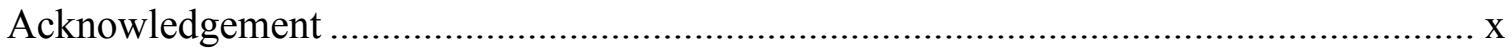

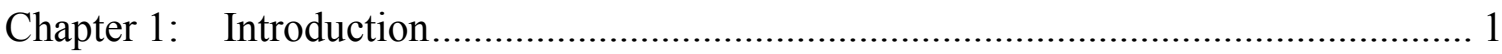

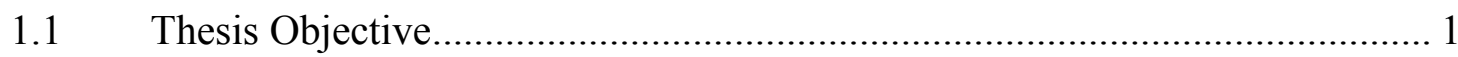

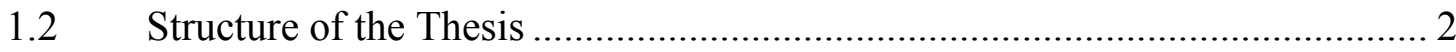

Chapter 2: Literature Review of Optimization Techniques for Harmonic Mitigation .... 3

$2.1 \quad$ Ant Colony System (ACS).......................................................................... 3

2.2 Honey Bees Mating Optimization (HBMO) …………................................. 8

2.2.1 Honey Bees Modeling.................................................................... 9

2.3 Particle Swarm Optimization (PSO) .............................................................. 11

2.3.1 PSO Modelling............................................................................. 12

$2.4 \quad$ Artificial Neural Networks (ANN) ………….......................................... 13

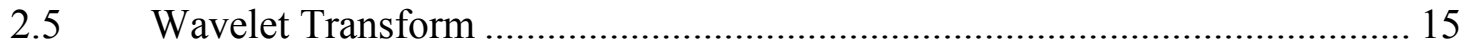

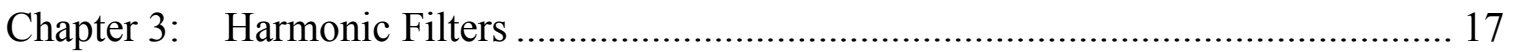

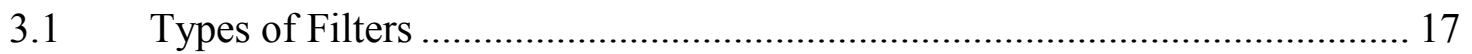

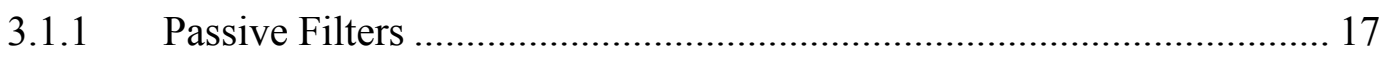

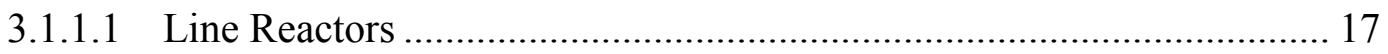

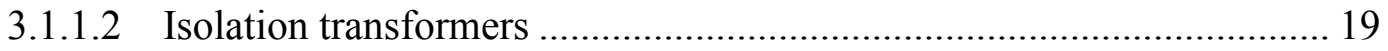

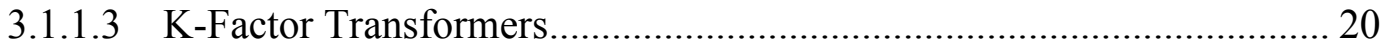

3.1.1.4 Tuned Harmonic Filters (THF) ............................................................ 21

3.1.1.5 Isolated Gate Bipolar Transistors (IGBT) Based Fast Switched

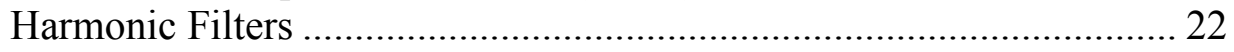

3.1.1.6 Low Pass Harmonic Filters (LPHF) ..................................................... 23

3.1.1.7 12\&18 Pulse Rectifiers .................................................................... 24

3.1.1.8 Active Filters............................................................................. 25

3.2 Literature Review for Harmonic Filters....................................................... 26

Chapter 4: Harmonic Power Flow for Radial Distribution Systems ............................. 33

4.1 Backward/Forward Sweep Algorithm for RDS ............................................ 33 
4.2 Harmonic Power Flow Algorithm ......................................................... 38

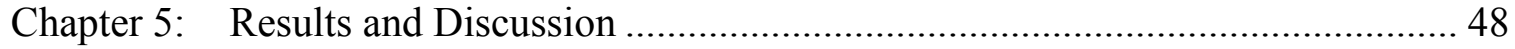

$5.1 \quad$ Implementation of Ant Colony System Algorithm .................................... 50

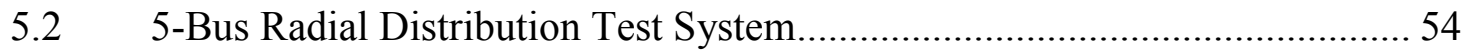

5.2.1 Harmonic Load Flow Results Before Filter Placement ........................ 55

5.2.2 Harmonic Load Flow Results Before Filter Placement ....................... 55

5.3 11-Bus Radial Distribution Test System................................................ 59

5.3.1 Harmonic Load Flow Results Before Filter Placement ........................ 61

5.3.2 Harmonic Load Flow Results After Filter Placement.......................... 62

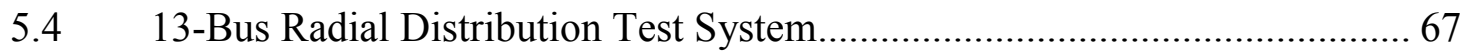

5.4.1 Harmonic Load Flow Results Before Filter Placement ........................ 69

5.4.2 Harmonic Load Flow Results After Filter Placement........................... 70

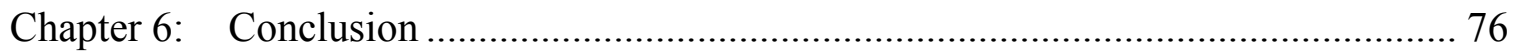

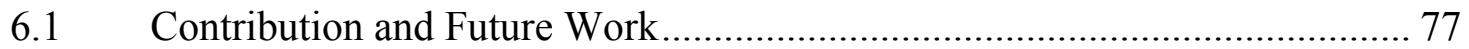

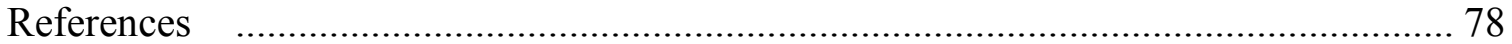




\section{List of Tables}

Table 1: Typical K-Factor vs. effective impedance (6-pulse load) [26] 21

Table 2: Advantages and disadvantages of low pass filter 24

Table 3: 5-bus system bus and line data 54

Table 4: Harmonic Load flow results for 5-bus 55

Table 5: Ant Colony results for 5-bus system 55

Table 6: Harmonic Load flow results for 5-bus after filter placement 56

Table 7: Harmonic Load flow results for 5-bus after filter placement 58

Table 8: 11-bus system bus and line data $\quad 60$

Table 9: Harmonic Load flow results for 11-bus 61

Table 10: Ant Colony results for 11-bus system 62

Table 11: Harmonic Load flow results for 11-bus after filter placement 63

Table 12: Harmonic Load flow results for 11-bus after filter placement 66

Table 13: 13-bus system bus and line data 68

Table 14: Harmonic Load flow results for 13-bus 69

Table 15: Ant Colony results for 13-bus system 70

Table 16: Harmonic Load flow results for 13-bus after filter placement 71

Table 17: Harmonic Load flow results for 13-bus after filter placement 74 


\section{List of Figures}

Figure 1: The HBMO algorithm [16] 9

Figure 2: Algorithm and computational flowchart [16] 10

Figure 3: Search method of PSO [20] 12

Figure 4: The mammalian neuron $\quad 13$

Figure 5: General structure of the neural network feed forward system 14

Figure 6: Line Reactor [29] 18

Figure 7: Isolation transformer [30] 19

Figure 8: Connection of tuned filter [31] 21

Figure 9: Frequency response of tuned filter [27] 22

Figure 10: Connection of LPHF [31] 23

Figure 11: 12-pulse rectifier (left), 18-pulse rectifier (right) [27] 25

Figure 12: 12-pulse rectifier (left), 18-pulse rectifier (right) [27] 25

Figure 13: Single line diagram of n-bus RDS 33

Figure 14: Single line diagram of n-bus unbalanced RDS 39

Figure 15: 5-bus radial distribution system 54

Figure 16: Ant colony output for 5-bus 56

Figure 17: THD before and after filter placement for 5-bus system 57

Figure 18: Vrms before and after filter placement for 5-bus system 57

Figure 19: Ploss before and after filter placement for 5-bus system 58

Figure 20: 11-bus radial distribution system 59

Figure 21: Ant colony output for 11-bus 62

Figure 22: THD before and after filter placement for 11-bus system 64

Figure 23: Vrms before and after filter placement for 11-bus system 65

Figure 24: Ploss before and after filter placement for 11-bus system 66

Figure 25: 13-bus radial distribution system $\quad 67$

Figure 26: Ant colony output for 11-bus 70

Figure 27: THD before and after filter placement for 13-bus system 72

Figure 28: Vrms before and after filter placement for 13-bus system 73

Figure 29: Ploss before and after filter placement for 13-bus system 74 


\begin{abstract}
This thesis presents an application of the Ant Colony algorithm for optimizing filter placement and sizing on a radial distribution system to reduce power losses and keep the effective harmonic voltage values and the total harmonic distortion (THD) within prescribed limits. First, a harmonic load flow (HLF) algorithm is performed to demonstrate the effect of harmonic sources on total power loss. Then the Ant Colony algorithm is used in conjunction with HLF to place a selection of filter sizes available at each possible location so that both power loss and THD are minimized. As a result the optimal adjustment of location and size of the filter are determined. Results of computational experiments on standard test systems are presented to demonstrate improvement and effectiveness of using the filters at the optimal location. The methodology used can be easily extended to different distribution network configurations.
\end{abstract}




\section{List of Abbreviations and Symbols Used}

\begin{tabular}{|c|c|}
\hline ACS & Ant Colony System \\
\hline $\mathrm{ACO}$ & Ant Colony Optimization \\
\hline APFs & Active Power Systems \\
\hline AR & Amplitude Ratio \\
\hline VTHD & Voltage Total Harmonic Distortion \\
\hline THD & Total Harmonic Distortion \\
\hline $\mathrm{HPF}$ & Harmonic Power Flow \\
\hline GBDT & Generalized Benders Decomposition Theory \\
\hline CLF & Constrained Load Flow \\
\hline RL & Reinforcement Learning \\
\hline $\mathrm{OPF}$ & Optimal Power Flow \\
\hline ISTN & Inherent Structure Theory Network \\
\hline SI & Sensitivity Index \\
\hline NSI & Normalized Sensitivity Index \\
\hline DHSE & Distribution Harmonic State Estimation \\
\hline GA & Genetic Algorithm \\
\hline SG & Smart Grids \\
\hline MPSO & Modified Particle Swarm Optimization \\
\hline HBMO & Honey Bees Mating Optimization \\
\hline $\mathrm{AB}$ & Artificial Bee \\
\hline $\mathrm{ANN}$ & Artificial Neural Network \\
\hline WLS & Weight Least Square \\
\hline TLS & Total Least Squares \\
\hline DGs & Distributed Generators \\
\hline MV & Medium Voltage \\
\hline RDS & Radial Distribution System \\
\hline RDPF & Radial Distribution Power Flow \\
\hline
\end{tabular}




$\begin{array}{ll}\text { KCL } & \text { Kirchoff's Current Law } \\ \text { KVL } & \text { Kirchoff's Voltage Law } \\ |\mathrm{Bi}| & \text { Magnitude of the ith Branch Current } \\ \mathrm{Ri} & \text { ith Branch Resistance } \\ \mathrm{nb} & \text { Number of Branches in RDS } \\ \mathrm{Vo} & \text { Initial Bus Voltage Vector } \\ \text { ACSA } & \text { Ant Colony Search Algorithm } \\ \text { EPI } & \text { Effective Percent Impedance }\end{array}$




\section{Acknowledgement}

All thanks to Allah (God) who gives and grants me this success and this degree. I am very grateful to my sponsor Technical \& Vocational Training Corporation (TVTC) for giving me this opportunity to study abroad and continue my Master's degree. I would also like to thank my parents, my wife, my brothers, sisters and all my family for supporting and encouraging me.

Additionally, I am deeply thankful to my supervisor, Dr. M. El-Hawary, for his advice and guidance throughout my study.

I would also like to acknowledge Najran College of Technology and all my fellow colleagues. Finally, I would like to appreciate the support of all my friends and colleagues at Dalhousie University. 


\section{Chapter 1: Introduction}

As a result of the increasing penetration of energy from renewable sources into the power grid, power quality has currently become a recurring problem. The cause of such problems can be attributed to two main sources, the injected harmonics from renewable energy sources and abnormal operating conditions. Some of the generated power is lost due to harmonic injections of different elements at each stage of a distribution system. Harmonics result from the presence of harmonic producing equipment that causes overheating and malfunctioning of those devices; these are undesirable as they cause power losses and also affect the voltage profile. The voltage drop also occurs at distribution feeder due to variations in load demand and many times it falls under the workable operating limits. The unequal loading phases can also make distribution systems less effective.

It would be less expensive and time saving if the harmonics and the effects of abnormal operating conditions could be reduced. In order to work efficiently, the distribution system needs to be upgraded. Low pass filters can solve this problem if optimized by using an effective technique [1,2].

\subsection{Thesis Objective}

Harmonic filters like low pass harmonic filters are commonly used elements in distribution systems to improve power quality by enhancing voltage profiles. The placement and sizing of these filters play a critical role not only in improving the power quality but reducing the total generation cost. Recently some Heuristic techniques have proven reliable in solving such optimization problems. Ant Colony Optimization is one 
such technique. Hence, this thesis focuses on reducing the total power loss and suppressing the effect of total harmonic distortion caused by harmonics present in the system by optimally placing and sizing the low pass harmonic filter using Ant Colony algorithm.

\subsection{Structure of the Thesis}

Chapter 1, the introduction is followed by Chapter 2 which presents a brief description of optimization techniques and a literature review of the proposed methodologies for harmonic reduction. Different harmonic filter types are discussed along with a literature review in Chapter 3. The radial distribution load flow and harmonic load flow algorithms are discussed in Chapter 4. Chapter 5 presents results and discussions. Chapter 6 offers the conclusion and suggestions for future work. 


\section{Chapter 2: Literature Review of Optimization Techniques for Harmonic Mitigation}

Different optimization techniques are available nowadays (heuristic and nonheuristic techniques). This chapter is focused on the optimization techniques that were used before for dealing with the best size and location of the harmonic filters to improve the output performance of the voltage waveform. Some of these techniques are described, in the following order: Ant Colony Optimization (ACO), Honey Bees Mating Optimization (HBMO), Particle Swarm Optimization (PSO), Artificial Neural Networks (ANN), and Wavelets Processing.

\subsection{Ant Colony System (ACS)}

The Ant Colony System (ACS) was inspired by the foraging behavior of ants. Biologists have determined that ants always find the shortest distance between food and their nests because they leave pheromone trails, which allows them to communicate with each other and transfer information about paths to food. The search for food generally begins by a group of ants choosing random paths. During the initial searches, they leave constant density trails as to their movements in time. Subsequently, the density of the trails on the shorter paths increases and assists them in finding the shortest route to food. The ant colony algorithm, which parallels this ant behavior, begins a search among a population by measuring the competence of individual members of the population based on a cost function until the individuals all converge to an optimum [3].

Sirjani and Hassanpour [4] used the ant colony algorithm for the placement and sizing of shunt capacitors in order to reduce the power losses in the system. Widely 
distributed shunt capacitors in the distribution network are used to reduce power loss by compensating reactive power. Installation of shunt capacitors, however, depends on the placement and sizing of the shunt capacitors. First a forward/backward substitution method was used to calculate the total power loss. It was used as an objective function for ant colony algorithm to optimally place an appropriately sized shunt capacitor.

Bouri and Zeblah in $[5,6]$ used the ant colony algorithm for shunt capacitor allocation in Radial Distribution Systems (RDS). The allocation of capacitors is considered a medium voltage (MT) reactive power balancing procedure. Constraints are imposed by limits on capacitor switching transients. The constraints are very important when a station capacitor bank is used along with pole mounted capacitors. An ACS algorithm was employed to determine the optimum placement of capacitors in a way that economic advantages, power and energy loss reduction were maximized. The system was tested on the Algerian Company of Electricity and Gas, and was successful in the optimal allocation of 25 pole mounted capacitors in 9 different distribution systems, with 50 loads supplied through radial distribution feeders.

Annaluru [7] used a multi-level ACS for solving the capacitor placement and sizing problem. In this method, the cost function was calculated using the Newton-Raphson power flow method. Furthermore, Ghorbani and Hosseinian in [8] presented in their study the ACS algorithm for optimal distribution of loads among feeders to reduce losses. Because distribution lines are subject to a mixture of residential commercial and industrial loads, parts of the distribution system may become more heavily loaded at certain times. Load currents can thus be transferred between feeders, and the load current can be rescheduled in a more efficient way to avoid the overload and minimize losses. 
The least expensive approach is to reconfigure the distribution system by changing the status of network switches. Generally, artificial ants are able to optimize a solution by using information in the form of pheromones that are deposited in the path. The study proposed a new strategy for switch selection by the ants, and the results were compared to three different networks. The optimum solution was found by using the state transition rule, local updating, and global updating rule and they indicated an efficient reduction in real power losses.

Huang in [9] used ACS \for hydroelectric generation scheduling. For hydroelectric power generation to be considered economical, scheduling is a crucial factor. The main objective of scheduling is to minimize the fuel cost of thermal units by determining the optimal amount of generated powers for the hydro units. A standard problem of hydro scheduling is subject to some electric power balance, hydro discharge limits and reservoir limits. Generally a good hydro schedule aims at reducing the production cost, improving system reliability and maximizing the energy capabilities of reservoirs. In their paper an algorithm based on ant colony system was studied and proposed to enhance the scheduling of hydroelectric generation. The ACS was applied to solve the problem by first determining the search space of multi spaced scheduling. Next, using ants which refer to a collective of cooperative agents, a near optimal solution can be achieved to solve the scheduling problem. In the algorithm three rules were used: the state transition rule, the local pheromone updating rule and the global pheromone updating rule to facilitate the computations, thereby enhancing the optimization capabilities in a significant way. This approach, which was tested on the Taiwan power system, demonstrated the effectiveness of this method. 
Vlachogiannis in [10] used an ACS based algorithm to solve constrained load flow problem (CLF). First the ACS algorithm was compared with Q-learning and probabilistic CLF method. Furthermore, a 14-bus system was studied and the ACS algorithm was used to control reactive power in order to minimize real power losses. The results showed that the ACS algorithm provides the optimal results in less time as compared to the other two methods. The proposed algorithm was also found flexible and applicable for multi criteria optimization problems.

The operation, planning and control of electrical energy systems present many economical and technical problems to electrical utilities. A major problem is the determination of a secure way of short term generation scheduling and dispatch. Among the various approaches that have been used, dynamic programming and Lagrangian relaxation based algorithms have received wide attention. In recent times ANN and genetic algorithms have been widely used. Simulating foraging behavior of ants, the paper used the ant colony search algorithms to solve short term generating scheduling problems of power systems, with the main goal of investigating its applicability in power system optimization. In the algorithm the cooperating agents (ants) cooperate in finding a good solution to the scheduling problem. The effectiveness of the algorithm is demonstrated on a model power system and the outcome compared to those obtained from a conventional hybrid DP type of scheduling method. The results of this comparison showed that in economic terms, this ACSA method could be applied successfully to short term generation scheduling problems [11].

Furthermore, Nikoukar and Gandomkar in [12] elaborated that a capacitor placement problem within a network includes the finding of suitable sizes and optimal 
locations to place them. This is a combinational optimization problem where the search space has a size equal to $(k+1)^{n}$, where $\mathrm{n}=$ number of buses on the network and $\mathrm{k}=$ possible number of capacitors. The paper presents a new approach to capacitor selection problem based on the ACS by modeling capacitor optimization problem as a discrete one. In this approach, the algorithm maintains two separate tables of pheromones, and stochastic solutions are generated by the ants based on these tables. Periodic updates of the pheromone tables lead to even better solutions. The author concludes that this method is an effective approach to capacitor placement optimization within a distribution network [12].

Alvarado [13] presented a study in which he used an ACS for solving the electrical distribution planning problem. The method was a modification of the ant system algorithm that allowed artificial ants to determine the optimal route from pheromone deposits from different trajectories. The algorithm chose the optimum network plan to satisfy demand requirements. Fixed and variable costs associated with future investments and power losses are all included in the objective function of this algorithm. The system was tested on 139-node network in Ecuador with "adequate" results and was shown as a "flexible" tool for distribution system planning engineers [13].

Ziari and Jalilian in [14] implemented an algorithm based on Ant Colony System for allocation and sizing of 12 active power filters (APF). The APF used in the paper was harmonic current injecting sources. The modeling of APF was done as a current source having real and imaginary parts and an objective function was created by adding weighted maximum total harmonic distortion and total current. An ant colony system was implemented using the objective function for the allocation and sizing of APF. The 
objective of the algorithm was to minimize voltage total harmonic distortion (VTHD) and total APF current injection. The algorithm was tested on a 6-bus system. The ideal case was the placement of APF on all buses with non-linear loads which fully compensates the harmonic distortion. In the second case APFs were placed on all 6 buses. The results showed a small discrepancy of $13 \%$ between total APF and non-linear load currents.

\subsection{Honey Bees Mating Optimization (HBMO)}

Search algorithms based on honey bee mating have been considered as metaheuristic for typical swarm-based approaches to optimization. In this study, the Honey Bee Mating Optimization (HBMO) is compared to a "nonlinear, continuous constrained problem with continuous decision and state variables to demonstrate the efficiency of the algorithm in handling the single reservoir operation optimization problems". The author shows that the HBMO is comparable to traditional linear programming methods such as LINGO 8.0 based on the promising results exhibited by the HBMO.

A honey bee colony can be compared to a large city with different sectors. The queen is the most important entity in the hive whose job is to keep the city going by producing new queens and worker bees. The queen mates with about 18 drones once in her life, storing the sperm of each drone in a pouch. The drones die off after mating. The queen uses the stored sperm to fertilize her eggs, producing queens, drones and workers. Whether a bee would become a queen, drone or worker depends on the queen fertilizing the eggs.

The queen lives on a diet of royal jelly which is secreted by "nurse bees" from their glands and is fed to the queen alone, making her bigger than any other bee in the hive. Though the queen may live for up to 6 years, workers and drones never live for more than 
six months. The queen and the drones take a mating flight where she mates in midair with as many of the drones as possible. After the mating flight, the drones are forced out of the hive to conserve food for the queen and other worker bees [15].

\subsubsection{Honey Bees Modeling}

Breeding begins when the queen returns from her mating flight. The queen selected for her relative fitness, mates with randomly selected sperm from her spermatheca (also called receptaculum seminis (plural: receptacula seminis), is an organ of the female reproductive tract in insects) and produces a required number of broods. Workers are selected in proportion to their ability to improve the brood. Broods are sorted according to their fitness, and the best brood replaces the worst queen until no broods better than the queen are available. The remaining brood is then killed off and a new mating flight initiated until all convergence criteria, in this case, all assigned mating flights, are met or completed. Figure 1 shows the main steps in the HBMO algorithm. Figure 2 shows a full scale computational flow chart that clarifies the algorithm and translates biological terminology of honey bee mating into technical terminology [16].

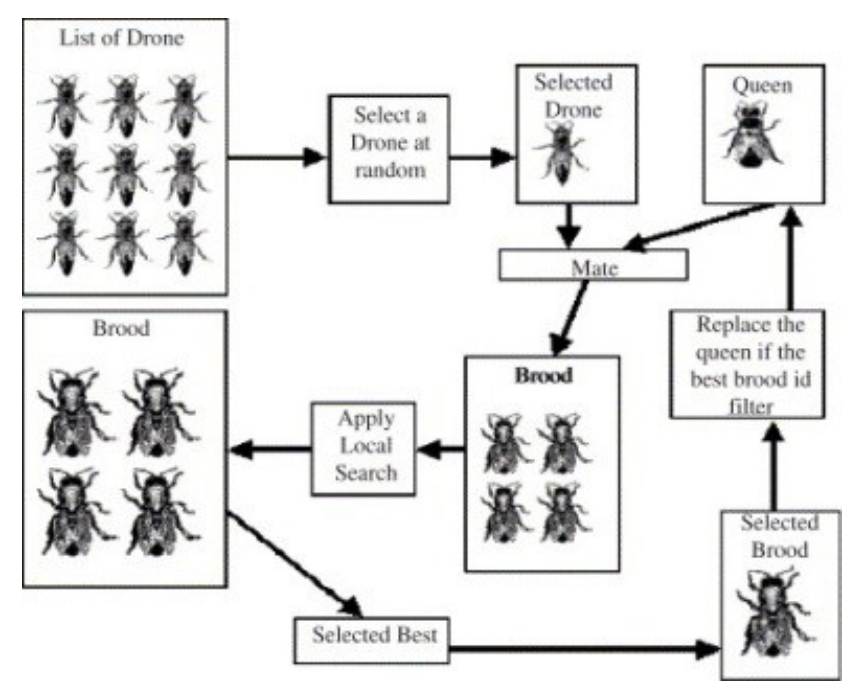

Figure 1: The HBMO algorithm [16] 


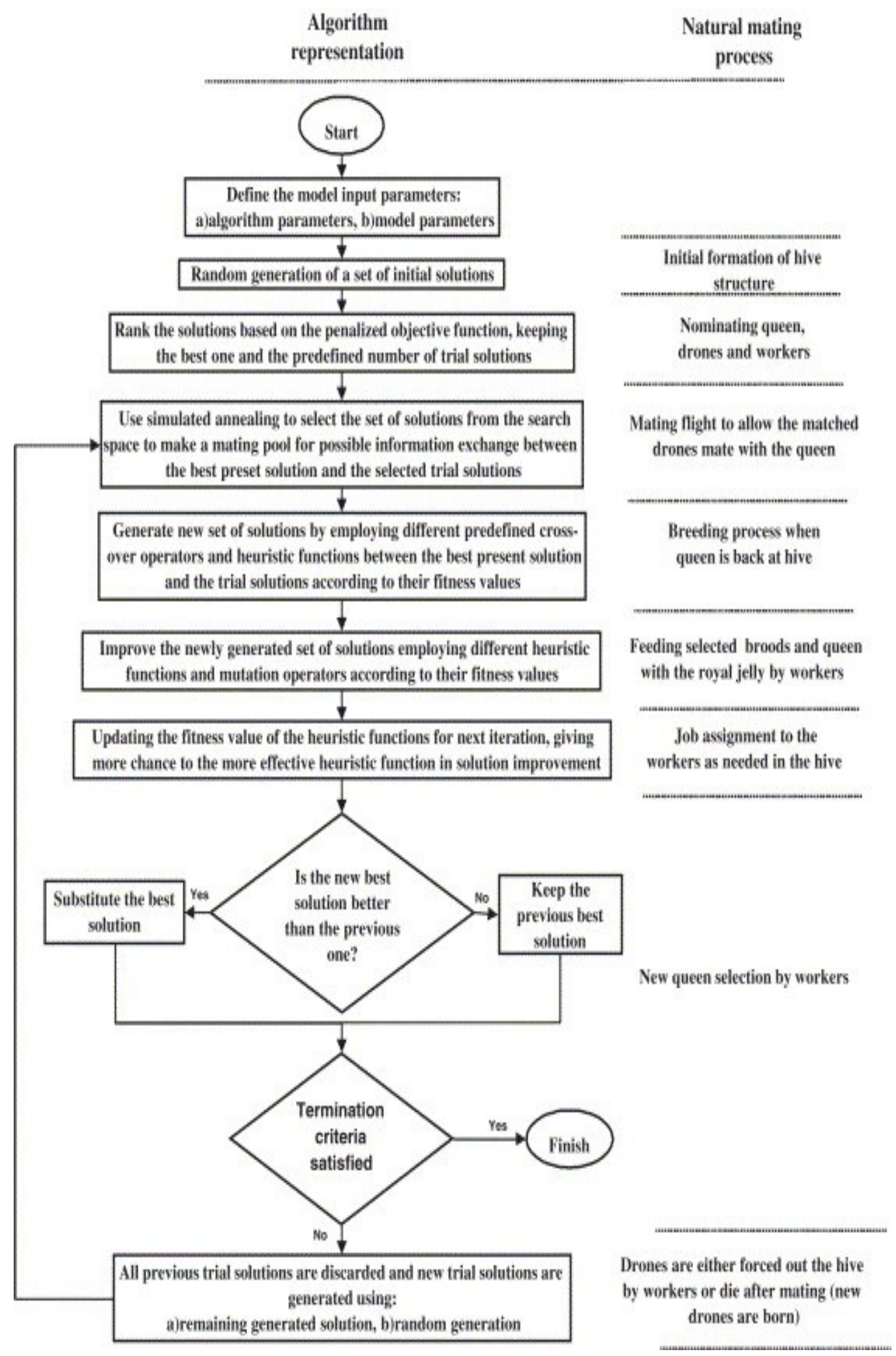

Figure 2: Algorithm and computational flowchart [16] 
If the mating-flight between queen and drones is considered a set of transitions in a state space (the environment) then the queen moves between different states with some velocity and mates probabilistically with the drones encountered at each stage. The queen is initialized at the start of the flight with some energy content and then after the mating when her spermatheca, the pouch within which she stores sperm is full, her energy is within some threshold from zero. In the development of this algorithm, worker bee functionality is restricted to brood care, and thus are presented as heuristic, acting to improve or take care of a set of broods. These may include feeding the future queen with her royal jelly.

\subsection{Particle Swarm Optimization (PSO)}

Particle swarm optimization (PSO) is a meta-heuristic optimization technique introduced by Kennedy and Eberhart [17] in 1995. Like the ACS and HBMOs described above, PSO is also a population based algorithm which is inspired by bird flocking and fish schooling. PSO was originally designed to solve continuous nonlinear optimization problems but more research has been done which has made it possible for PSO to handle constrained nonlinear optimization problems with both discrete and continuous variables [18]. Arefi and Haghifam [19] presented a modified PSO (MPSO) algorithm to solve distributed harmonic state estimation problem. Eajal and El-Hawary [20] introduced a

modern PSO technique for the optimal placement of shunt capacitors for unbalanced radial distribution system. 


\subsubsection{PSO Modelling}

The PSO algorithm is described briefly. First a population called a swarm is generated randomly. The swarm consists of many individual particles. Each particle present in the swarm represents a potential solution of the problem considered. All the particles move through a D-dimensional search space at a random velocity. Finally in accordance with the following equations every individual particle updates its velocity and position to give the most optimal solution [20]:

$$
\begin{gathered}
V_{i}^{(k+1)}=\chi\left(V_{i}^{(k)}+c_{1} \times \operatorname{rand}_{1} \times\left(\text { Pbest }_{i}-X_{i}^{(k)}\right)+c_{2} \times \text { rand }_{2} \times\left(\text { Gbest }_{i}-X_{i}^{(k)}\right)\right) \\
X_{i}^{(k+1)}=X_{i}^{(k)}+V_{i}^{(k)}
\end{gathered}
$$

$V_{i}^{(k)}$ : velocity of ith particle at time $\mathrm{k}$

$X_{i}^{(k)}:$ position of $\mathrm{i}$-th particle at time $\mathrm{k}$

$\chi:$ the inertia weight

Pbest $_{i}$ : the best value of ith particle so far

Gbest $_{i}$ : the best value among Pbest $_{i}$ so far

$\operatorname{rand}_{1}$ and $\operatorname{rand}_{2}:$ random variable between 0 and 1

$c_{1}$ and $c_{2}$ : constants

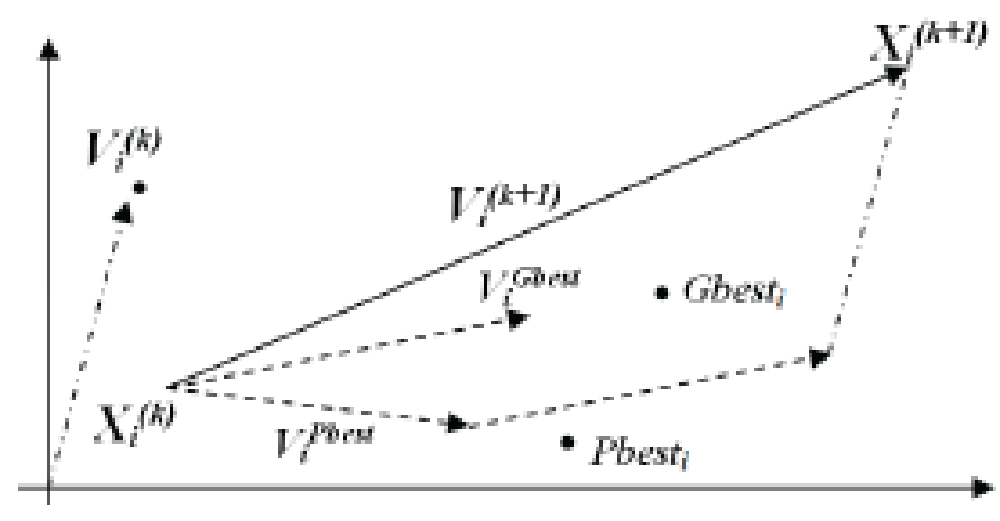

Figure 3: Search method of PSO [20] 


\subsection{Artificial Neural Networks (ANN)}

Artificial Neural Networks (ANN) are mathematical models of natural neural networks in the human brain which consist of billions of interconnected neurons. ANN attempts to simulate brain functions such as the ability to compute, speech, recognition of faces and control activities. The human brain is composed of more than 10 billion interconnected neurons, each cell can receive, process, and transmit information. The network of brain nerve fibre is called dendrites and they are connected to the cell body at the cell nucleus. The axon, a single long fibre emanating from the cell body, branches into strands and sub-strands, which then connect to other neurons through synapses. Figure 4 shows a mammalian neuron. When the neurons receive a signal, they activate and emit a signal through the axons. If the signal is strong, the potential reaches a threshold and a pulse is sent through the axon firing the cell in the process. Figure 5 shows the network structure of the neural network feed system.

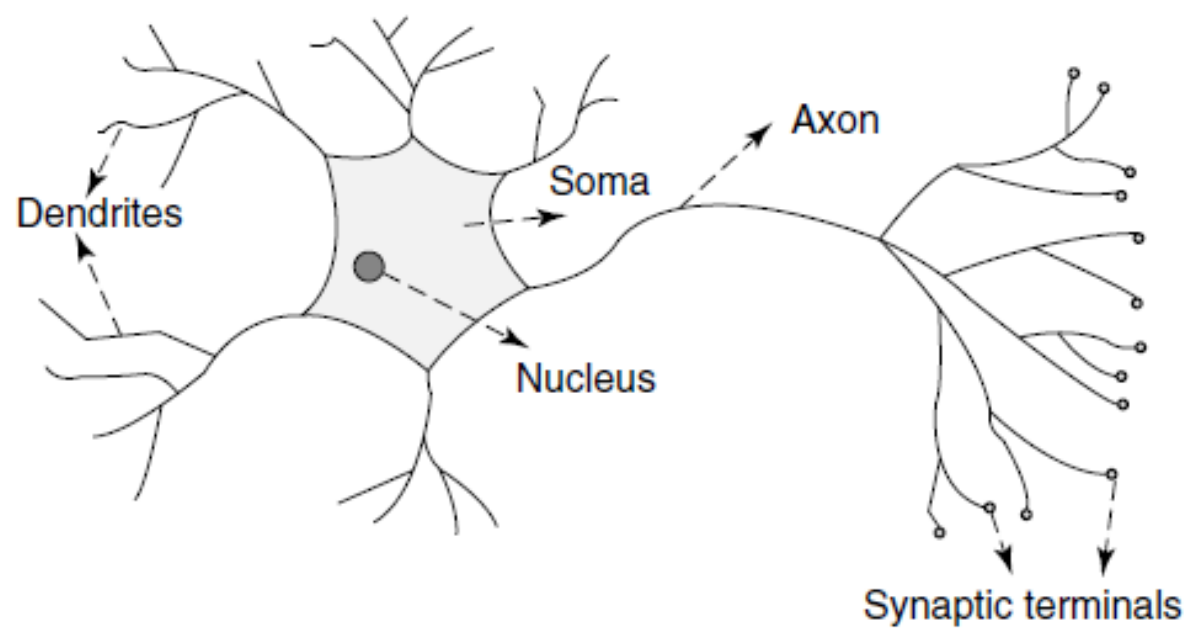

Figure 4: The mammalian neuron 


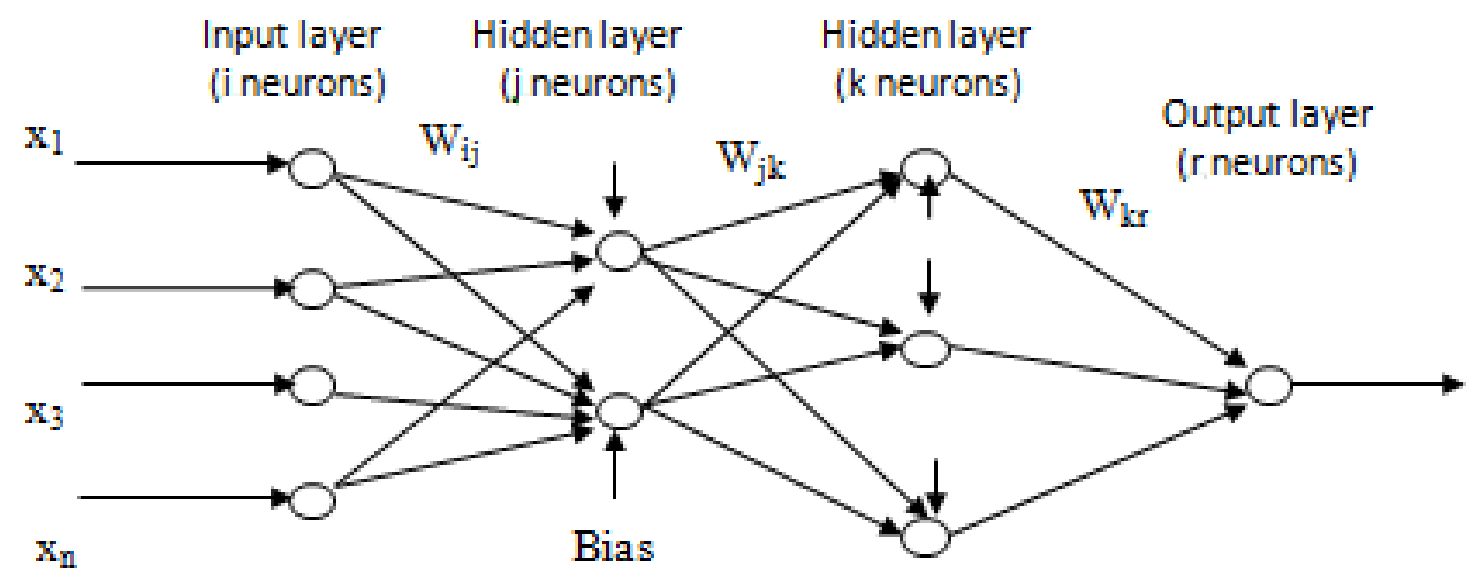

Figure 5: General structure of the neural network feed forward system

In ANNs the effects of the synapses are simulated by connection weights that modulate the incoming, or input, signal effect. The non-linear characteristics of neurons are represented by the transfer function. The weighted sum of the input signals is equal to the impulse of the neurons. ANNs are commonly used in forecasting where accuracy results from the increase in data input.

Neural networks must be configured so as to produce a desired set of outputs given a certain set of inputs. One method of configuration is to set the weights by using a priori knowledge, or train the neural network in inputting teaching patterns and then allowing the network to change the weights according to additional learning rules. Each neuron's output can be expressed as a function of the input signals as [21]:

$$
\begin{array}{ll}
\mathrm{Y}_{\mathrm{j}}(\mathrm{t})=f\left(\sum_{i=1}^{x} W_{i j} X_{i}(t) \pm b_{i}\right) & \text { for } \mathrm{j}=1, \ldots . \mathrm{H}_{1} \\
\mathrm{Y}_{\mathrm{k}}(\mathrm{t})=f\left(\sum_{j=1}^{\mathrm{H} 1} W_{j k} Y_{j}(t) \pm b_{i}\right) & \text { for } \mathrm{k}=1, \ldots . \mathrm{H}_{2} \\
\mathrm{O}_{\mathrm{r}}(\mathrm{t})=f\left(\sum_{r=1}^{\mathrm{H} 2} W_{k r} Y_{k}(t) \pm b_{i}\right) & \text { for } \mathrm{r}=1, \ldots \mathrm{Z}
\end{array}
$$


$\mathrm{Y}_{\mathrm{j}}(\mathrm{t}) \& \mathrm{Y}_{\mathrm{k}}(\mathrm{t})=$ Quantity computed by the first and second hidden neurons respectively

$\mathrm{O}_{\mathrm{r}}(\mathrm{t})=$ Network output

$X \& Z=$ Number of input and output neurons

$\mathrm{H}_{1} \& \mathrm{H}_{2}=$ Number of first and second hidden neurons

$\mathrm{W}_{i j}, \mathrm{~W}_{\mathrm{jk}} \& \mathrm{~W}_{\mathrm{kr}}=$ Adjustable weights between respective layers

$b_{i}=$ Biases

$f=$ Transfer function

\subsection{Wavelet Transform}

Barros and Diego [22], in their study, presented an overview of different methods used in the analysis of harmonic distortion using wavelets. Wavelet transforms are used in estimating harmonic, inter-harmonic and sub-harmonic distortions. They are also used for tracking time varying harmonics and have applications in harmonic compensation. In Fournier analysis, waveforms with short duration, zero means and sharp decay to zero at both ends of the wave are used instead of stationary sinusoidal waves. To vary the timefrequency resolution, the short waveforms are shifted and translated. Because waveforms are able to adapt the window size, the results of the analysis of this kind of non-stationary signals is improved.

The first to analyze wavelet transform and the capacity to analyze non-stationary signals in power systems was P. F. Ribero [23, 24] who presented wavelet theory's basic concepts and the potential for its application in power system distortion. Santoso et al. [25] used wavelets to detect and localize distortions in power quality including fast, long and slow voltage variations and harmonic distortion. Short wavelet functions were 
proposed for solving different cases of transients. Robertson et al.[26] used wavelet transforms for power system transients, separating power into different components. He described a methodology, initially for capacitors switching and fault transients, for constructing a transient recognition system that separated transient power signals into a number of components for further processing. 


\section{Chapter 3: Harmonic Filters}

There are many types of filters available for harmonic reduction. Some three phase mitigation equipment available include line reactors, isolation transformers, K-factor transformers, tuned harmonic filters, IGBT based fast switched harmonic filters, low pass harmonic filters, $12 \& 18$ pulse rectifiers and active filters. In this thesis low pass filter is used to reduce losses and suppress distortion caused by harmonics.

\subsection{Types of Filters}

Today there are many filter types available, and they are classified into two types, passive and active filters.

\subsubsection{Passive Filters}

Passive filter uses passive elements. It is constructed using resistors, capacitors and inductors. There are different types of passive filters and they are described in this section.

\subsubsection{Line Reactors}

Line reactors are connected in series with non-linear loads like an ASD. They are the simplest and lowest cost means to attenuate harmonics. When a series reductive reactance is inserted into a line reactor circuit, the harmonics are attenuated and voltage transients are absorbed therefore preventing a voltage source ASD from tripping as a result of over-voltage. The effective impedance of the reactor in relation to the load determines the actual spectrum of harmonics and the magnitude of the harmonic distortion. 


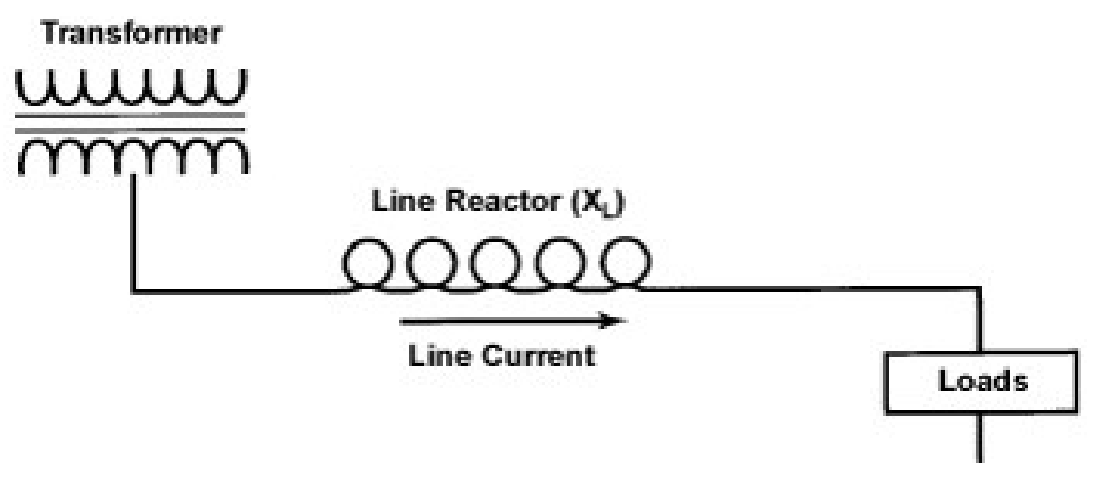

Figure 6: Line Reactor [29]

In line reactors, the type of rectifier and the effective source impedance determine both the magnitude and the phase angle of the input current harmonic distortion. The ratings on the name plates of line reactors and main supply transformers indicate per unit impedance relative to the rated full load current. The rate of reduction of the actual load current is proportional to the effective impedance reduction. A line reactor rated at $5 \%$ impedance, appears as only $2 \%$ in a load that draws $50 \%$ of the rated current.

The total voltage drop across the reactor, or transformer (input circuit impedance) provides the percent input impedance relative to the given load. This voltage drop is caused by current flow from the fundamental load through the impedance in comparison to system voltage. This relation is shown in Equation 3.1:

$$
\text { \%Impedence }=\frac{I_{f} X_{f} \sqrt{3}}{V_{L-L}} 100
$$

$I_{f}=$ Fundamental current

$X_{f}=$ Reactance at fundamental frequency

$V_{L-L}=$ Line to line voltage (rms) 


\subsubsection{Isolation transformers}

Isolation transformers are effective ways of attenuating harmonic distortion, especially because the magnitude of the harmonics presents in, and flowing to, the load is determined in a major way by the circuit impedance. Harmonics get attenuated when the leakage inductance of isolation transformers gives appropriate values of the circuit impedance. Delta primary and wye secondary form the most common configuration of isolation transformers.

In these transformers, the inductive reactance easily passes the fundamental current because it is low enough at the fundamental frequency. An electrostatic shield between primary and secondary windings can be supplied to isolation transformers causing the creation of a low impedance path to attenuate harmonics, noise, transients and zero-sequence currents. Further, the capacitive coupling of windings and shield helps in the attenuation of common mode disturbances to the originating side of the transformer with the wye secondary providing an electrical ground for the load circuit. Following, Figure 7 shows an isolation transformer:

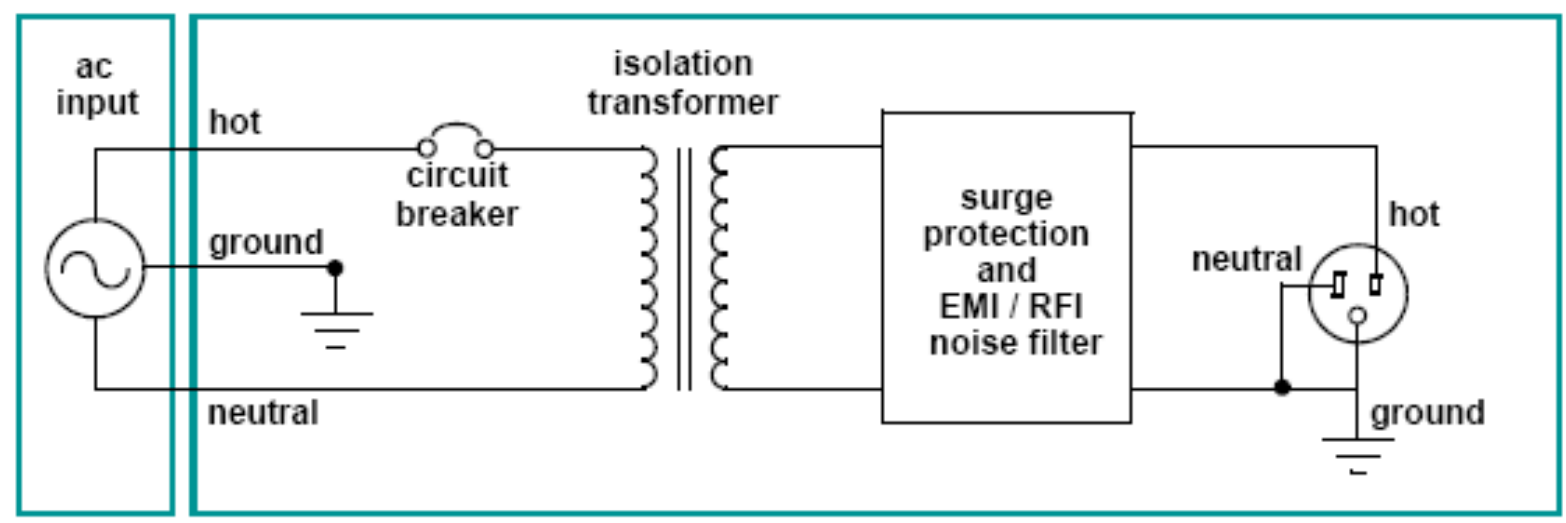

Figure 7: Isolation transformer [30] 


\subsubsection{K-Factor Transformers}

There are two categories of transformer losses: eddy current losses and 12-R losses. 12-R losses are based on the RMS current. Both these losses are proportional to the square of the currency and frequency. The design of the K-Factor transformer is meant to mitigate the fundamental frequency losses as well as the rise in temperature caused by current harmonics within the transformer windings. A K-Factor of 8 , for example, implies that the transformer will be able to handle heat generation due to eddy current losses 8 times better than a non K-Factor transformer [28].

K-Factor transformers differ in design from other transformers, especially in the current carrying capacity of phase conductors and in the transformer windings. The neutral bus has twice the current carrying capacity that phase conductors possess, while the windings generally have multiple insulated conductors, which are designed to reduce the skin effect caused by the current harmonics within the windings. Further, in K-Factor transformers, there is lower flux density in the magnetic core than in non K-Factor transformers.

The K-Factor, which is a function of the magnitude of harmonic current and harmonic order, is computed from a waveform harmonic spectrum. When the THD is made up of high order harmonics like those of 12 and 18 pulse rectifiers, a low THD will not necessarily be an indication of a low K-Factor. This means that THD measurements should be taken if there is suspicion of high order harmonics in current waveform. Table 2 shows K-Factors and the corresponding waveforms and harmonic spectra. These are given for typical values for the effective percent impedance (EPI) of the input circuit. 
Table 1: Typical K-Factor vs. effective impedance (6-pulse load) [27]

\begin{tabular}{|c|c|c|c|c|c|c|c|}
\hline \%Impedance & 0.5 & 1 & 1.5 & 2 & 3 & 4 & 5 \\
\hline K-Factor & 21 & 14 & 11 & 9 & 6.8 & 5.6 & 4.8 \\
\hline
\end{tabular}

When the EMI is $2 \%-5 \%$, a transformer with a K-Factor of 9 can supply individual non-linear loads. With a mix of linear and non-linear loads, a new K-Factor requirement is often lower than when it is only supplied by a non-linear load. When nonlinear loads are supplied by the transformer, and are connected line to neutral, harmonics occur in multiples of three (triplen). On the primary side of the circuit, harmonic currents can be mitigated by the delta connection, especially if the load current has a high level third harmonic. On the secondary side, between the phase and neutral conductors, triplen harmonics would occur. Triplen harmonics sum up algebraically because they are in phase with each other relative to the phase conductors.

\subsubsection{Tuned Harmonic Filters (THF)}

THFs are composed of both inductive and capacitive elements connected in series to form tuned LC circuits. THFs have wide applications within the facility where they are employed either within the service entrance or in distribution transformers. Figure 8 shows a THF connected as a shunt devise to the power system and Figure 9 shows its frequency response.

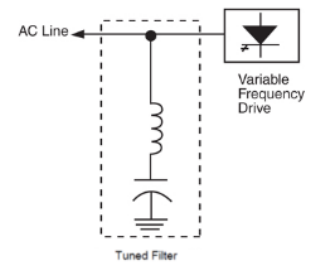

Figure 8: Connection of tuned filter [31] 


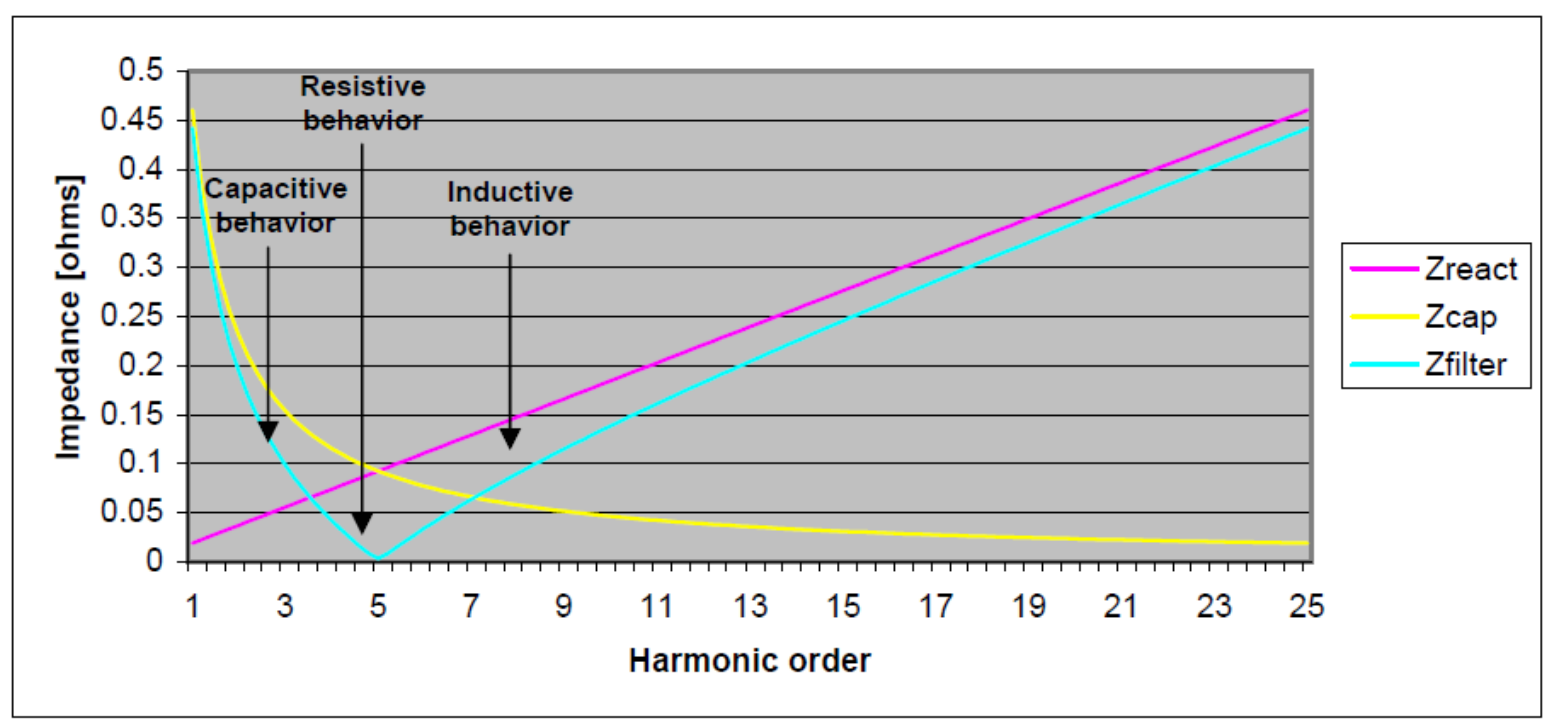

Figure 9: Frequency response of tuned filter [27]

\subsubsection{Isolated Gate Bipolar Transistors (IGBT) Based Fast Switched Harmonic Filters}

When there is a need for reactive power supply and mitigation of harmonics, a fast switched harmonic filter, which rapidly switches between IN and OUT using IGBTs instead of contactors becomes the solution. Automatic filters are useful when reactive power changes occasionally without accompanying extreme rapid changes in load conditions. When there are rapidly changing demands for reactive power though, these automatic filters do not respond quickly enough, hence the need for IGBT based filters to maintain acceptable harmonic distortion levels or acceptable power factors. The major advantage of this filter is that it can respond to dynamically changing load conditions in real time without discharging the capacitors, which may create voltage spikes. The fastswitched filter has the performance of typical tuned filters, which can mitigate total current harmonic distortions of $3 \%-12 \%$. 


\subsubsection{Low Pass Harmonic Filters (LPHF)}

A passive filter is a kind of filter that is made only from passive components like inductors or capacitors; a low-pass filter is a filter that allows low-frequency signals to pass and attenuates higher frequency signals. LPHFs are able to achieve low levels of harmonic distortion and attenuate all harmonic frequencies and guarantee good results. Figure 10 shows a typical LPHF configuration with tuned shunt elements plus one or more series elements. Overall system harmonics are reduced when the series elements increase input effective impedance, and the shunt circuit is detuned. The shunt circuit which is relative to both the load side and the supply end of the filter prevents the infiltration of harmonics from the same transformer or feeder as well as preventing resonance of these harmonic frequencies. Most of the other circuit harmonics can be removed just from the manner of the shunt elements tuning. The LPHF allows a hybrid combination of shunt and series elements. When these elements are applied there may not be the need to even perform system harmonic analysis.

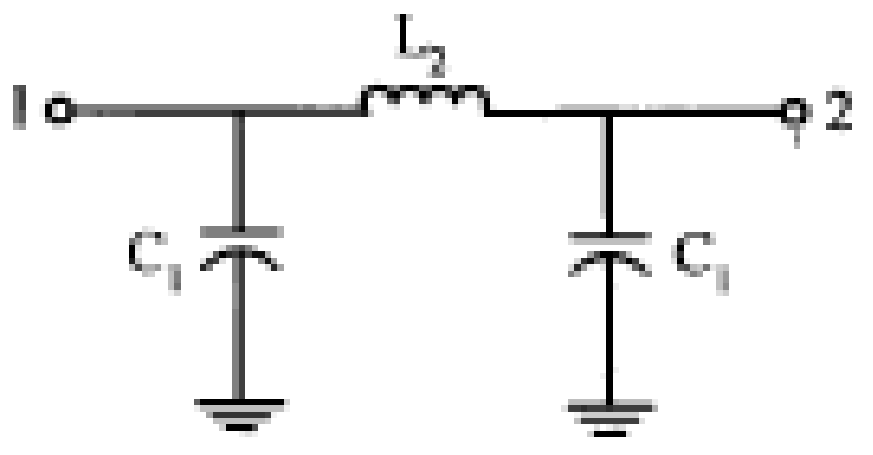

Figure 10: Connection of LPHF [31]

In a series connection of an LPHF ahead of a 6-pulse rectifier load for instance, very little distortion occurs at the input side of the filter but significant current and voltage distortion occur at the output stage where the load is connected. As a result it is 
recommended that only non-linear loads be connected. Overheating and low life expectancy result when linear loads are connected to the output stage of the filter. Under loaded conditions there is a voltage drop at the filter as a result of series reactance, but under no load conditions, there is a voltage boost because of the shunt capacitor and reactor. Even though the LPHF is regulated at $5 \%$ typically, there is normally a $10 \%$ boost with some low pass filters. Some limitations to the use of low pass filters are that some may not be suitable for use in eighth silicon controlled rectifiers and may require external reactors to be installed to the line or load line for effective harmonic mitigation.

Table 2: Advantages and disadvantages of low pass filter

\begin{tabular}{|l|l|}
\hline \multicolumn{1}{|c|}{ Advantages } & \multicolumn{1}{c|}{ Disadvantages } \\
\hline - Relatively low cost & - Must connect in series with the load \\
- Low residual harmonics & - Can only be used with non-linear \\
- Predictable Results & loads \\
- No analysis or harmonic studies & - Experience low (leading) power \\
required & factor at light loads \\
\hline
\end{tabular}

\subsubsection{12\&18 Pulse Rectifiers}

12 and 18 pulse rectifiers, unlike the filters above, are not ad-on solutions because of the internal changes that the manufacturer must add to the input rectifier section. Figure 11 show this rectifier and transformer configuration. 

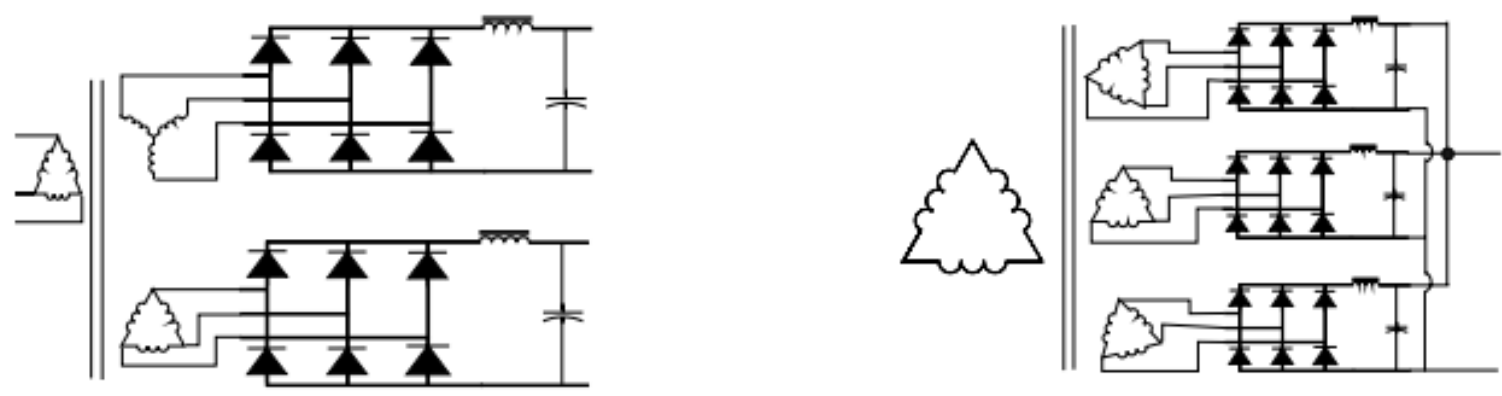

Figure 11: 12-pulse rectifier (left), 18-pulse rectifier (right) [27]

Equation 3.2 identifies the harmonics present in the input current harmonic spectrum for 3 phase power systems and rectifiers.

$$
h=k P+/-1
$$

$\mathrm{k}$ is an integer $(1,2,3$, etc.) and $\mathrm{P}$ is the number of rectifier pulses on the dc bus waveform for one cycle of ac input voltage (it is also the number of individual rectifiers used in the rectifier).

\subsubsection{Active Filters}

One of the newest technologies that exist for harmonics mitigation is active filter. An active filter uses active components such as an amplifier. In contrast with low pass filter it requires an external power source to operate. Active filtering techniques can either be applied as a standalone harmonic filter or can be incorporated with power electronics equipment. The application of an active filter is illustrated in Figure 12.

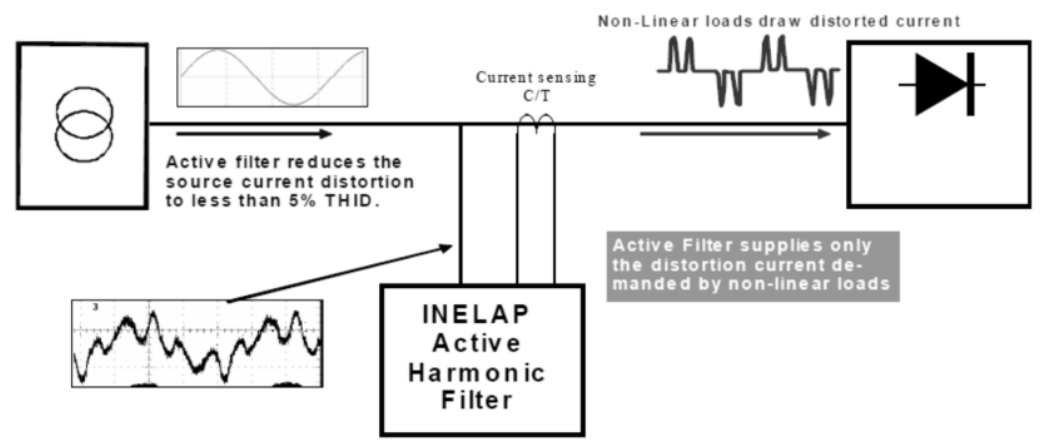

Figure 12: 12-pulse rectifier (left), 18-pulse rectifier (right) [27] 
Typically, an active filter injects the appropriate inverse currents to cancel individual harmonics by monitoring the load currents, filtering out the fundamental frequency currents and analyzing the frequency and magnitude content of the remainder. Active filters can achieve harmonic distortion levels as low as 5\% THD-I or less and are able to normally cancel harmonics up to about the 50th harmonic. Furthermore, the maintenance requirement of active filters can be higher than passive filter solutions as they utilize power electronics circuitry. In addition, the inherent losses associated with active filters also have a tendency to be higher as compared to passive filter solutions [27].

\subsection{Literature Review for Harmonic Filters}

The proliferation of nonlinear loads has increasingly injected harmonic currents into power systems, interacting with the system impedance to cause voltage distortion in network buses [32-33]. To achieve better control of harmonic voltage distortion, Chang and $\mathrm{Chu}$ in [34] proposed an approach to find filter candidate buses quickly. The planning problem was formulated as one of constrained optimization, which was then solved for the optimal size of the filter component. They used single-tuned passive filters with direct search algorithm for the purpose of controlling distortion in the whole power system.

$\mathrm{Wu}$ and Chiang in [35] investigated harmonic amplification problems in a plastic plant and the appropriate mitigation methods. Many harmonic amplifications problems have been caused by single tune filters, especially when the frequencies of parallel resonance were located at harmonic orders. This study discussed such problems in a distribution plant where 5th, 7th, 11th, and 13th order single tuned filters had been 
installed to mitigate the distortion caused by three 6-pulse rectifiers. The study found that after reduction of capacitance in the filter capacitors the frequency of the 8th order harmonic, and its harmonic current were amplified. The study found many failures concerning the capacitors, and recommended 2 high pass damped filters to replace the single-tuned filters. The effect of this would correct amplification problems at even order harmonics. The authors suggest a method of adjusting single-tuned filters to 17 th and 35 th order high pass damped filters. The paper was motivated by an event in the pumped storage power station in Taiwan when the THD of the current at the lightening feeder reached up to $184 \%$, and burned out power fuses in the process.

Zaveri and Chudasama in [36] presented control strategies for harmonic mitigation and power factor correction using shunt active filter under various source voltage conditions. The study stated that the quality of power within the distribution system was "polluted" as a result of a power burden that was reactive, load currents that were harmonically "contaminated" and unbalanced. They employed a shunt active filter (SAF) that was shunted with the load regulating the injection of current into the system in an efficient way under both ideal and non-ideal source voltage conditions. The authors deemed their approach to be a novel one that was based on an "improved instantaneous active and reactive power component method". They compared their proposed controlled method with othermethods: the instantaneous reactive power theory, the symmetrical component theory and the dg theory. The performance of their proposed strategy was evaluated in terms of compensation in the reactive power, source current reduction and harmonic compensation. The authors found that under conditions of distorted source voltage there was superior performance from the SAT "improved instantaneous active 
and reactive current compensation theory". They presented simulations results from a complete model of SAF.

Zaveri and Chudasama in [37] introduced a Current Source Converter-Based Active Power Filter for mitigation of harmonics at the interface of distribution and transmission systems. This study addressed distribution companies, commercial and industrial plants and customers, who had power demands and had raised the mean power factors by the installation of shunt compensation systems as a result of the requirements of power compensation in regulation. Shunt-connected contactor-switched plain capacity banks have been the most popular solution to the reactive power compensation problem. In this study a medium power current source converter (CSC-based shunt active power filter APF) was designed to suppress low order harmonic amplification. The APF was implemented at the medium voltage interface bus between the distribution and transmission systems. The design was a result of the large shunt capacity banks that were installed in this interface to compensate for reactive power. In the study four CSC based APF modules, operated in parallel, and were connected to a medium voltage bus via a coupling transformer. Switching ripples were eliminated by the LC type input filter while harmonic frequencies at the input filter were suppressed. The selective harmonic amplification method was applied to the APF in the study and acted as a temporary connection to a problematic interface bus, pending permanent solutions for that location.

Panda and Mikkili in [38] FLC based shunt active filter ( $p-q)$ and (Id-Iq) control strategies for mitigation of harmonics with different fuzzy MFs using MATLAB and realtime digital simulator. Electricity customers' demand for good quality power supply had made it challenging to grid operators because of the increase in sensitive loads. 
Conventional load compensation, using passive filters, had draw backs like resonance overloading and detuning, were not suitable for fast changing loads. Shunt active power filters (SAPF) became an effective way of alleviating such problems. The authors posited that a selection of controlled strategies for the shaft is significant in getting the desired compensation that one seeks from the system. Load compensation and instantaneous power and current (p-q and Id-Iq) are two main control strategies that are used. The problems of power quality have an adverse effect economically. In this study different control strategies that were used in the extraction of three phase reference currents for shunt active power filters were compared. The performance of these stagiest were evaluated under different source conditions. The criteria for the evaluation were harmonic mitigation and dc-link voltage regulation. The shaft that was proposed by the authors gains outstanding compensation abilities and was able to eliminate uncertainty within the distribution system

Guerin and Miegeville in [39] performed optimal placement and sizing of harmonic filters aboard an electric propulsion ship through simulated annealing. Simulated annealing, a method that was extrapolated from the annealing process of iron was used in this article to derive an optimization technique for harmonic mitigation. The idea was to find the best placements of harmonic filters within a system, not to cancel the harmonic voltages but to reduce them with respect to the fixed limits. In order to reduce harmonic distortion, harmonic filters were placed within a system. The extent of the reduction distortion depended on the placement of these filters within the system, so filter location, types and sizes became crucial to distortion reduction. Prior work had focussed on capacity placement for "reactive power compensation" but very little work had been 
done on filter placement" for voltage distortion" In this study, the problem of harmonic voltage reduction was reformulated to minimise the current that passes through the filtering system. Optimizing of size of the filter was applied for each filter using the simulated annealing process.

Annealing is a process of cooling down molten iron or steel in stages slowly, until it reaches the stable or lowest energy state. The simulated algorithm is modelled after the metallurgical process. The authors stated that an advantage of this system was that it allowed problems of harmonic distortion to avoid a local solution and obtain a global optimal solution. This allowed the best placement of filters in a power system with minimum power size. Out of this the optimization technique, a software package had been created and tested in some real power systems. From one of the cases, they concluded that for a power system of similar size as the case study, a best solution could be found by some considerations like cost of filter installation, available space on the ship, and the power expected.

Chang and Wang in [40] used passive filters in a power system for controlling voltage distortion. Harmonic filter placement was studied in this research by an approach that correlated the inherent structure theory network and harmonic current injections at busses throughout the distribution network. These busses were characterized as either with minimum total harmonic voltage reduction, THDv, or the bus where the placement of filters would result in maximum THDv. This approach had the advantage of providing some insights into network structure and harmonic current sources with regards to harmonic filter performance. 
Furthermore, the article acknowledged the source of harmonic distortions as the constant growth of non-linear loads in customer network installations. These individual nonlinear loads included fluorescent lighting, computers and peripheral devices, office machines, central air conditions and heat pumps. Single-tuned passive filters had become the most economic and effective way of reducing the harmonic distortions of these nonlinear loads. In this approach, a Sensitivity Index (SI) based on ISTN was used to determine the capacitor busses for placing harmonic filters. Then a new normalized SI was formed which included the effect of the harmonic current on the harmonic filters.

The paper presented 3 approaches, two based on the SI and the other on average harmonic current emission level. The results indicated that if a highly sensitive network bus had high harmonic current emissions then they were effective for placement of harmonic filters.

Bhattacharya and Deng in [41] used an active filter for harmonics elimination in solar photovoltaic grid connection and standalone systems. As a result of the development of new grid codes, the output signal harmonic control of power converters had become very important in medium and high power applications. Smaller fast switching insulated gate bipolar transistors (IGBT) had become the latest in converters because of transistors technology development. Electrical applications that used high power needed to have their energy systems integrated into the distribution grid. Tuned filters became a way to minimize harmonics while decreasing the weight and total cost of the system. The IEEE Standard.519, revised in 1992 provided direction to deal with voltage and current distortion that was a result of non-liner modes and static power converters. Normally, the grid quotes would define the maximum tolerated THD but the 
IGBT based power drivers themselves generated harmonics which were translated into the power system. These harmonics could easily be eliminated by passive LC or RLC filters, which unfortunately, had their own limitations. This study used fast Fourier transforms analysis to analyse the harmonics that were generated by the IGBT converter. In this application photovoltaic arrays were connected to converters and the utility grid, and a second order Butterworth system showed that THD could be reduced to $32.59 \%$ to $1.59 \%$.

Chou and Liu in [42] presented an optimal planning method of large passive harmonic filters set at high voltage level. The planning of harmonic filters was important if harmonic filtering and reactive power compensation were to be achieved. Planning helped to determine the types, set numbers and other parameters that the filters must have. The filters could be set at low or high voltage depending on whether non-linear loads or a filter with large capacity of up to 12 MVA was required. The study provided an optimum planning method for large passive harmonic filters based on "multi type and multi set" filters considering also the source impedance variation, filtering requirements and power factor improvement. The study focused on 4 types of filters: single-tuned, second order, third order and C-type damped filters for the planning. The optimization problem was formulated by the construction of cost function and filter constraints. The simulated annealing algorithm was then used to search for an optimal solution for the planning of the filters. The SA algorithm yielded best results in 3 test cases of filter planning. 


\section{Chapter 4: Harmonic Power Flow for Radial Distribution Systems}

There are several methods to perform power flow analysis. In this thesis the backward/forward sweep algorithm is utilized which is explained in detail as follows:

\subsection{Backward/Forward Sweep Algorithm for RDS}

Backward-forward sweep power flow algorithm for Radial Distribution Systems (RDS) developed by Teng [43] is used in this study. In this radial distribution power flow algorithm (RDPF), two matrices namely $[\mathrm{BIBC}]$ and $[\mathrm{BCBV}]$ are formed to get the power flow solution. [BIBC] is the relationship matrix between bus current injections and branch currents. Whereas, $[\mathrm{BCBV}]$ represent the relationship between branch currents and bus voltages. In order to explain the backward-forward sweep method a radial distribution system is considered having ' $n$ ' buses. The single line diagram is presented in Figure 13.

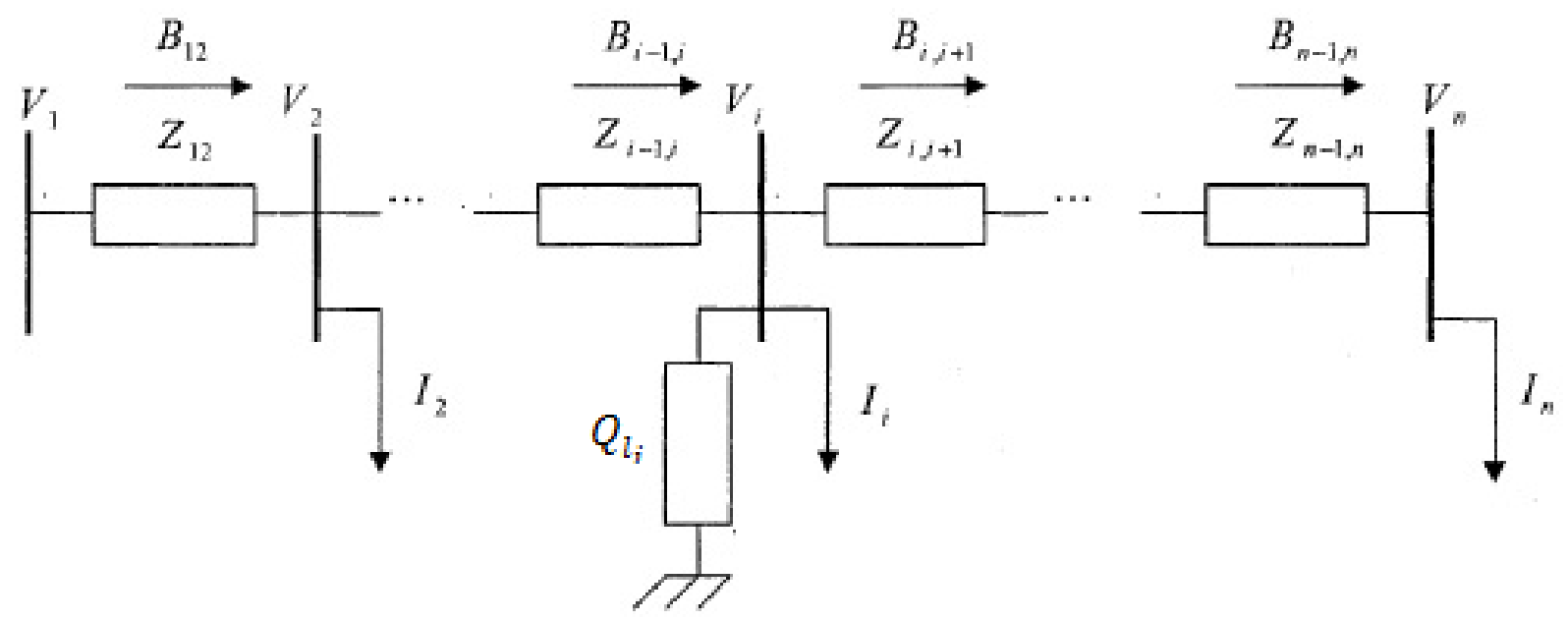

Figure 13: Single line diagram of n-bus RDS 
The e system is assumed to be balanced with (n-1) load buses which are treated as PQ buses and bus 1 is a slack/swing bus. The general bus ' $i$ ' current injection $\mathrm{k}^{\text {th }}$ iteration is given by the following equation:

$$
I_{i}^{k}=\left(\frac{S_{i}^{s c h}}{V_{i}^{k}}\right)^{*}
$$

where,

$S_{i}^{s c h}=$ the scheduled complex power at bus $\mathrm{i}$

$V_{i}^{k}=$ the bus ' $i$ 'voltage at the $\mathrm{k}^{\text {th }}$ iteration

When a filter is placed at bus ' $i$ ', the bus current injection is given by:

$$
I_{i}^{k}=\left(\frac{S_{i}^{s c h}}{V_{i}^{k}}\right)^{*}-\left(\frac{j Q_{l_{i}}}{V_{i}^{k}}\right)^{*}
$$

where,

$Q_{l_{i}}=$ the reactive power injection of filter placed at bus ' $i$ '

Now, the Kirchhoff's current law (KCL) is applied by backward current sweep starting from the last bus ' $n$ ' to the source bus 1 to obtain the branch currents. In this step we will obtain the first of the two matrices:

$$
B_{n-1, n}=I_{n}
$$

:

$$
B_{i, i-1}=I_{i+1}+I_{i+2}+\cdots+I_{n}
$$

$B_{i-1, i}=I_{i}+I_{i+1}+\cdots+I_{n}$

$$
B_{i-2, i-1}=I_{i-1}+I_{i}+I_{i+1}+\cdots+I_{n}
$$




$$
B_{12}=I_{2}+\cdots+I_{i-1}+I_{i}+I_{i+1}+\cdots+I_{n}
$$

Equations (4.3-4.6) may be written in a matrix form as follows:

$$
\left[\begin{array}{c}
B_{12} \\
\vdots \\
B_{i-2, i-1} \\
B_{i-1, i} \\
B_{i, i-1} \\
\vdots \\
B_{n-1, n}
\end{array}\right]=\left[\begin{array}{ccccccc}
1 & \ldots & 1 & 1 & 1 & \ldots & 1 \\
\vdots & \ldots & \ldots & \ldots & \ldots & \ldots & \vdots \\
0 & \ldots & 1 & 1 & 1 & \ldots & 1 \\
0 & \ldots & 0 & 1 & 1 & \ldots & 1 \\
0 & \ldots & 0 & 0 & 1 & \ldots & 1 \\
\vdots & \ldots & \ldots & \ldots & \ldots & \ldots & \vdots \\
0 & \ldots & 0 & 0 & 0 & \ldots & 1
\end{array}\right]\left[\begin{array}{c}
I_{2} \\
\vdots \\
I_{i-1} \\
I_{i} \\
I_{i+1} \\
\vdots \\
I_{n}
\end{array}\right]
$$

Equation 4.6 in a compact form is given as follows:

$$
[B]=[B I B C][I]
$$

where,

$[\mathrm{BIBC}]=$ the relationship matrix between branch currents and bus current injections

Now we will use the forward voltage sweep and apply Kirchhoff's voltage law (KVL) to obtain the bus voltages and second relationship matrix as follows:

$$
V_{2}=V_{1}-Z_{12} B_{12}
$$

$$
\vdots
$$$$
V_{i-1}=V_{i-2}-Z_{i-2, i-1} B_{i-2, i-1}
$$$$
V_{i}=V_{i-1}-Z_{i-1, i} B_{i-1, i}
$$

$V_{i+1}=V_{i}-Z_{i, i+1} B_{i, i+1}$

$$
V_{n}=V_{n-1}-Z_{n-1, n} B_{n-1, n}
$$


Equations (4.8-4.10) are written in terms of the slack/swing bus voltage $\left(V_{1}\right)$ as follows:

$$
V_{2}=V_{1}-Z_{12} B_{12}
$$

$\vdots$

$V_{i-1}=V_{1}-Z_{12} B_{12}-\cdots-Z_{i-2, i-1} B_{i-2, i-1}$

$V_{i}=V_{1}-Z_{12} B_{12}-\cdots-Z_{i-2, i-1} B_{i-2, i-1} Z_{i-1, i} B_{i-1, i}$

$V_{i+1}=V_{1}-Z_{12} B_{12}-\cdots-Z_{i-2, i-1} B_{i-2, i-1} Z_{i-1, i} B_{i-1, i}-Z_{i, i+1} B_{i, i+1}$

$\vdots$

$V_{n}=V_{1}-Z_{12} B_{12}-\cdots-Z_{i-2, i-1} B_{i-2, i-1} Z_{i-1, i} B_{i-1, i}-Z_{i, i+1} B_{i, i+1}$

$$
-\cdots-Z_{n-1, n} B_{n-1, n}
$$

Equations (4.11-4.13) are written in a matrix form as follows:

$$
\left[\begin{array}{c}
V_{2} \\
\vdots \\
V_{i-1} \\
V_{i} \\
V_{i+1} \\
\vdots \\
V_{n}
\end{array}\right]=\left[\begin{array}{c}
V_{1} \\
\vdots \\
V_{1} \\
V_{1} \\
V_{1} \\
\vdots \\
V_{1}
\end{array}\right]-\left[\begin{array}{ccccccc}
1 & \ldots & 1 & 1 & 1 & \ldots & 1 \\
\vdots & \ldots & \ldots & \ldots & \ldots & \ldots & \vdots \\
0 & \ldots & 1 & 1 & 1 & \ldots & 1 \\
0 & \ldots & 0 & 1 & 1 & \ldots & 1 \\
0 & \ldots & 0 & 0 & 1 & \ldots & 1 \\
\vdots & \ldots & \ldots & \ldots & \ldots & \ldots & \vdots \\
0 & \ldots & 0 & 0 & 0 & \ldots & 1
\end{array}\right]\left[\begin{array}{c}
B_{12} \\
\vdots \\
B_{i-2, i-1} \\
B_{i-1, i} \\
B_{i, i-1} \\
\vdots \\
B_{n-1, n}
\end{array}\right]
$$

y rearranging the above Equation 4.14, we obtain the change in voltage vector $[\Delta \mathrm{V}]$ as follows:

$$
[\Delta \mathrm{V}]=[\mathrm{BCBV}][\mathrm{B}]
$$

where, 
$[\mathrm{BCBV}]=$ the relationship matrix between bus voltages and branch currents

Hence, by substituting equation (4.7) in (4.15) $[\Delta \mathrm{V}]$ in terms of bus current injections is expressed as follows:

$$
\begin{gathered}
{[\Delta \mathrm{V}]=[\mathrm{BCBV}][\mathrm{BIBC}][\mathrm{I}]} \\
{[\Delta \mathrm{V}]=[D L F][\mathrm{I}]}
\end{gathered}
$$

where,

$[D I F]=$ the relationship matrix between voltage drops and bus current injections

As compared to Newton-Raphson load flow method, the RDPF solution is achieved by employing KCL and KVL respectively to form the matrices [BIBC] and $[\mathrm{BCBV}]$ and performing matrix multiplications. Hence, the time which is utilized for building Jacobean matrix, LU factorization, and backward/forward substitution required in Newton-Raphson method is avoided. Moreover, the following equations are solved iteratively to get the power flow solution:

$$
\begin{gathered}
I_{i}^{k}=\left(\frac{P^{s c h}+j Q^{s c h}}{V_{i}^{k}}\right)^{*} \\
{\left[\Delta \mathrm{V}^{k+1}\right]=[D L F]\left[I^{k}\right]} \\
{\left[V^{k+1}\right]=\left[V_{0}\right]-\left[\Delta \mathrm{V}^{k+1}\right]}
\end{gathered}
$$

where,

$\left[V_{0}\right]=$ initial bus voltage vector

The iterative process stops when the absolute of difference between previous bus current injection and most recent bus current injection is less than or equal to a prescribed tolerance $\varepsilon$ : 


$$
\left|I_{i}^{k+1}-I_{i}^{k} \leq \varepsilon\right|
$$

Finally, the total real power loss can be calculated by the following equation:

$$
\text { Ploss }=\sum_{i=1}^{b r} \text { Ploss }_{i}=\sum_{i=1}^{b r}\left|B_{i}\right|^{2} R \quad i=1,2, \ldots, b r
$$

where,

$\left|B_{i}\right|=$ magnitude of the ith branch current

$\mathrm{R}=$ the ith branch resistance

$b r=$ the number of branches in RDS

The total real power loss can be written in terms of [BIBC] as follows:

$$
\text { Ploss }=[R]^{T}|[B I B C][I]|^{2}
$$

\subsection{Harmonic Power Flow Algorithm}

The harmonic power flow algorithm (HPF) was introduced by Teng in [44]. Backward-forward sweep technique is also employed here but the effect of harmonics is also considered. In this study, harmonic sources of order $3^{\text {rd }}, 5^{\text {th }}, 7^{\text {th }}, 11^{\text {th }}, 13^{\text {th }}$ and $15^{\text {th }}$ are used. Each source generates these harmonics and multiple sources are placed at different nodes in the RDS.

The backward sweep in HPF algorithm is used to obtain the relationship matrix [A] which represents the relationship between branch currents and bus current injections for

the $h^{\text {th }}$ harmonic order, on the other hand the forward sweep generates the relationship matrix [HA] which gives the relationship between harmonic bus voltages and harmonic bus current injections. To explain, the harmonic power flow algorithm, an unbalanced nbus RDS, is considered and the single line diagram is demonstrated as follows: 


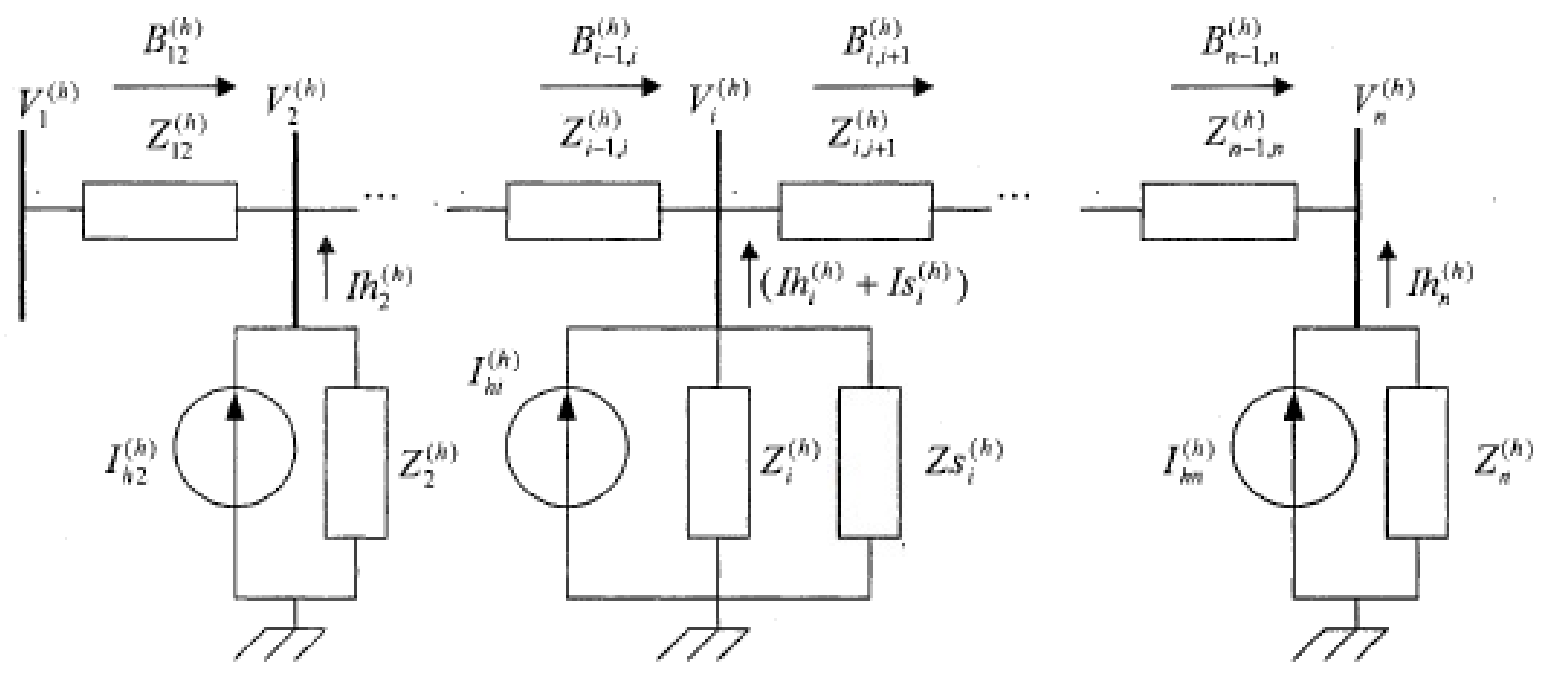

Figure 14: Single line diagram of n-bus unbalanced RDS

In the above figure, bus 1 is assumed to be the generator and is considered a slack bus; there are (n-1) load buses. The $h^{\text {th }}$ harmonic currents contributed by loads can be expressed as follows:

$$
\left[I h^{(h)}\right]=\left[I h_{2}^{(h)} \ldots I h_{i-1}^{(h)} I h_{i}^{(h)} I h_{i+1}^{(h)} \ldots I h_{n}^{(h)}\right]
$$

Also the harmonic current absorbed by filter which is connected at bus $i$ as shown in Figure 14.

$$
\left[I S^{(h)}\right]=\left[I s_{i}^{(h)}\right]
$$

It should be noted that the harmonic current vector of filter contains one element which is harmonic current absorbed by filter at bus $i$. The harmonic current vector of filter is $(n l \times 1)$ dimension for $n l$ filters placed in RDS. The $h^{\text {th }}$ harmonic bus current vector is:

$$
I^{(h)}=\left[\begin{array}{c}
I h^{(h)} \\
\vdots \\
I S
\end{array}\right]
$$

To find the current flowing in branches, backward current sweep is employed with KCL: 


$$
\begin{aligned}
& B_{n-1, n}^{(h)}=I h_{n}^{(h)} \\
& \vdots \\
& B_{i, i+1}^{(h)}=I h_{i+1}^{(h)}+\cdots+I h_{n}^{(h)} \\
& B_{i-1, i}^{(h)}=I h_{i}^{(h)}+I h_{i+1}^{(h)}+\cdots+I h_{n}^{(h)}+I s_{i}^{(h)} \\
& B_{i-2, i-1}^{(h)}=I h_{i-1}^{(h)}+I h_{i}^{(h)}+I h_{i+1}^{(h)}+\cdots+I h_{n}^{(h)}+I s_{i}^{(h)} \\
& \vdots \\
& B_{12}^{(h)}=I h_{2}^{(h)}+. .+I h_{i-1}^{(h)}+I h_{i}^{(h)}+I h_{i+1}^{(h)}+\cdots+I h_{n}^{(h)}+I s_{i}^{(h)}
\end{aligned}
$$

Equations (4.27-4.29) in a matrix form are given by:

$$
\left[\begin{array}{c}
B_{12}^{(h)} \\
\vdots \\
B_{i-2, i-2}^{(h)} \\
B_{i-1, i}^{(h)} \\
B_{i, i+1}^{(h)} \\
\vdots \\
B_{n-1, n}^{(h)}
\end{array}\right]=\left[\begin{array}{cccccccc}
1 & \ldots & 1 & 1 & 1 & \ldots & 1 & 1 \\
\vdots & \ldots & \ldots & \ldots & \ldots & \ldots & \ldots & \vdots \\
0 & \ldots & 1 & 1 & 1 & \ldots & 1 & 1 \\
0 & \ldots & 0 & 1 & 1 & \ldots & 1 & 1 \\
0 & \ldots & 0 & 0 & 1 & \ldots & 1 & 0 \\
\vdots & \ldots & \ldots & \ldots & \ldots & \ldots & \ldots & \vdots \\
0 & \ldots & 0 & 0 & 0 & \ldots & 1 & 0
\end{array}\right]\left[\begin{array}{c}
I h_{2}^{(h)} \\
\vdots \\
I h_{i-1}^{(h)} \\
I h_{i}^{(h)} \\
I h_{i+1}^{(h)} \\
\vdots \\
I h_{n}^{(h)} \\
I s_{i}^{(h)}
\end{array}\right]
$$

Equation 4.30 can also be expressed as follows:

$$
\left[B^{(h)}\right]=\left[A^{(h)}\right]\left[I^{(h)}\right]
$$

where,

$\left[A^{(h)}\right]=$ relationship matrix between bus current injection and branch current flows at $h^{\text {th }}$ harmonic

The $h^{\text {th }}$ harmonic branch current of a particular branch is given by: 


$$
B_{i j}^{(h)}=\left[A_{i j}^{(h)}\right]^{T}\left[I^{(h)}\right]
$$

where,

$\left[A_{i j}^{(h)}\right]=$ the coefficient vector of branch $(i-j)$ which is defined by:

$$
\left[A_{i j}^{(h)}\right]=\left[\begin{array}{c}
A h_{i j}^{(h)} \\
\cdots \\
A s_{i j}^{(h)}
\end{array}\right]
$$

where,

$\left[A h_{i j}^{(h)}\right]=$ the coefficient vector of branch $(i-j)$ due to the harmonic current flows of the loads through branch $(i-j)$

$\left[A s_{i j}^{(h)}\right]=$ the coefficient vector of branch $(i-j)$ due to the harmonic currents absorbed by the filter

Equation 4.33 can be expanded as follows:

$\left[A_{12}^{(h)}\right]=\left[A h_{12}^{(h)} \vdots A s_{12}^{(h)}\right]^{T}=\left[\begin{array}{llllllll}1 & \ldots & 1 & 1 & 1 & \ldots & 1 & \vdots\end{array}\right]$

$\vdots$

$\left[A_{i-2, i-1}^{(h)}\right]=\left[A h_{i-2, i-1}^{(h)} \vdots A s_{i, i+1}^{(h)}\right]^{T}=\left[\begin{array}{lllllll}0 & \ldots & 1 & 1 & 1 & \ldots & 1\end{array} \vdots 1\right]$

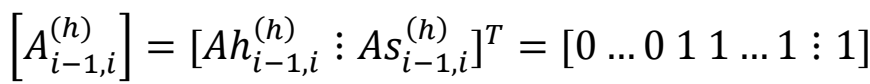

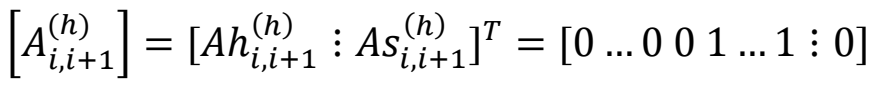




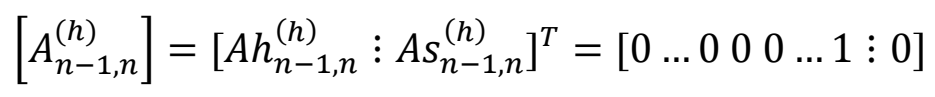

The harmonic voltage drop along the branch (1-2) in terms of the harmonic branch impedance and current is expressed as follows.

$$
\Delta \mathrm{V}_{12}^{(h)}=Z_{12}^{(h)} \mathrm{B}_{12}^{(h)}
$$

Equation 4.37 can be expanded as:

$$
\begin{aligned}
& \Delta \mathrm{V}_{12}^{(h)}=Z_{12}^{(h)}\left[A_{12}^{(h)}\right]^{T}\left[I^{(h)}\right]=Z_{12}^{(h)}\left(I h_{2}^{(h)}+. .+I h_{i-1}^{(h)}+I h_{i}^{(h)}+I h_{i+1}^{(h)}+\right. \\
& \left.\cdots+I h_{n}^{(h)}+I s_{i}^{(h)}\right)
\end{aligned}
$$

Similarly,

$$
\begin{aligned}
\Delta \mathrm{V}_{i-1, i}^{(h)} & =Z_{i-1, i}^{(h)}\left[A_{i-1, i}^{(h)}\right]^{T}\left[I^{(h)}\right] \\
& =Z_{i-1, i}^{(h)}\left(I h_{i}^{(h)}+I h_{i+1}^{(h)}+\cdots+I h_{n}^{(h)}+I s_{i}^{(h)}\right) \\
\Delta \mathrm{V}_{i, i+1}^{(h)} & =Z_{i, i+1}^{(h)}\left[A_{i, i+1}^{(h)}\right]^{T}\left[I^{(h)}\right]=Z_{i, i+1}^{(h)}\left(I h_{i+1}^{(h)}+\cdots+I h_{n}^{(h)}\right) \\
\vdots & \\
\Delta \mathrm{V}_{n-1, n}^{(h)} & =Z_{n-1, n}^{(h)}\left[A_{n-1,1}^{(h)}\right]^{T}\left[I^{(h)}\right]=Z_{n-1, n}^{(h)}\left(I h_{n}^{(h)}\right)
\end{aligned}
$$

Now, the forward voltage sweep and KVL is applied to calculate harmonic bus voltages as follows: 


$$
V_{2}^{(h)}=V_{1}^{(h)}-\Delta V_{12}^{(h)}
$$

where,

$V_{1}^{(h)}=$ the slack bus voltage at $h^{\text {th }}$ harmonic order

The slack bus voltage for the $h^{\text {th }}$ harmonic order is zero as the generator is supposed to supply voltage with a pure sinusoidal waveform. Hence,

$$
\begin{gathered}
V_{2}^{(h)}=Z_{12}^{(h)} I h_{2}^{(h)}+\cdots+Z_{12}^{(h)} I h_{i-1}^{(h)}+Z_{12}^{(h)} I h_{i}^{(h)}+Z_{12}^{(h)} I h_{i+1}^{(h)}+\cdots+Z_{12}^{(h)} I h_{n}^{(h)} \\
+Z_{12}^{(h)} I s_{i}^{(h)} \\
\vdots \\
V_{i-1}^{(h)}=Z_{12}^{(h)} I h_{2}^{(h)}+\cdots+\left(Z_{12}^{(h)}+\cdots+Z_{i-2, i-1}^{(h)} I h_{i-1}^{(h)}+\left(Z_{12}^{(h)}+\cdots\right.\right. \\
+Z_{i-2, i-1}^{(h)} I h_{i}^{(h)}+\left(Z_{12}^{(h)}+\cdots+Z_{i-2, i-1}^{(h)}\right) I h_{i+1}^{(h)}+\cdots+\left(Z_{12}^{(h)}\right. \\
+\cdots+Z_{i-2, i-1}^{(h)} I h_{n}^{(h)}+\left(Z_{12}^{(h)}+\cdots+Z_{i-2, i-1}^{(h)}\right) I s_{i}^{(h)} \\
\quad+\cdots+Z_{12}^{(h)} I h_{i-1}^{(h)}+\left(Z_{12}^{(h)}+\cdots+Z_{i-1, i}^{(h)} I h_{i}^{(h)}+\left(Z_{12}^{(h)}+\cdots\right.\right. \\
+Z_{i-1, i}^{(h)} I h_{i+1}^{(h)}+\cdots+\left(Z_{12}^{(h)}+\cdots+Z_{i-1, i}^{(h)}\right) I h_{n}^{(h)} \\
+\left(Z_{12}^{(h)}+\cdots+Z_{i-1, i}^{(h)} I s_{i}^{(h)} I h_{2}^{(h)}+\cdots+Z_{12}^{(h)} I h_{i-1}^{(h)}+\left(Z_{12}^{(h)}+\cdots+Z_{i-1, i}^{(h)} I h_{i}^{(h)}+\left(Z_{12}^{(h)}+\cdots\right.\right.\right. \\
+Z_{i-1, i}^{(h)}+Z_{i, i+1}^{(h)} I h_{i+1}^{(h)}+\cdots+\left(Z_{12}^{(h)}+\cdots+Z_{i-1, i}^{(h)}+Z_{i, i+1}^{(h)}\right) I h_{n}^{(h)} \\
V_{i+1}^{(h)}=Z_{12}^{(h)} I h_{2}^{(h)}+\cdots
\end{gathered}
$$




$$
\begin{aligned}
V_{i+1}^{(h)}=Z_{12}^{(h)} I h_{2}^{(h)} & +\cdots+Z_{12}^{(h)} I h_{i-1}^{(h)}+\left(Z_{12}^{(h)}+\cdots+Z_{i-1, i}^{(h)}\right) I h_{i}^{(h)}+\left(Z_{12}^{(h)}+\cdots\right. \\
& \left.+Z_{i-1, i}^{(h)}+Z_{i, i+1}^{(h)}\right) I h_{i+1}^{(h)}+\cdots+\left(Z_{12}^{(h)}+\cdots+Z_{i-1, i}^{(h)}+Z_{i, i+1}^{(h)}+\cdots\right. \\
& \left.+Z_{n-1, n}^{(h)}\right) I h_{n}^{(h)}
\end{aligned}
$$

Equations (4.43-4.45) can also be written as:

$$
\left[V^{(h)}\right]=\left[H A^{(h)}\right]\left[I^{(h)}\right]
$$

where,

$\left[V^{(h)}\right]=$ the harmonic bus voltages vector

$\left[H A^{(h)}\right]=$ the relationship matrix between the harmonic bus voltages and currents

The harmonic bus voltage of the filter is determined by:

$$
\left[V_{s}^{(h)}\right]=\left[H A_{s}^{(h)}\right]\left[I^{(h)}\right]
$$

where,

$\left[H A_{s}^{(h)}\right]=$ the row vectors of the matrix $\left[H A^{(h)}\right]$ associated with the bus at which filter is placed

The harmonic voltage of filter placed at bus $i$ in terms of its harmonic impedance is given as follows:

$$
V_{s_{i}}^{(h)}=-I_{s_{i}}^{(h)} \times Z_{s_{i}}^{(h)}
$$

where,

$I_{s_{i}}^{(h)}=$ the harmonic current of filter placed at bus $i$

$Z_{s_{i}}^{(h)}=$ the harmonic impedance of filter placed at bus $S_{i}$ 
Substituting Equation 4.48 into Equation 4.47, we get:

$$
-I_{s_{i}}^{(h)} \times Z_{s_{i}}^{(h)}=\left[H A_{s}^{(h)}\right]\left[\begin{array}{c}
I h^{(h)} \\
\vdots \\
I S^{(h)}
\end{array}\right]
$$

where,

$I h^{(h)}=$ the harmonic current vector of loads

$I S{ }^{(h)}=$ the harmonic current vector of the filter

The RDS assumed consists of $(n-1)$ loads and a single phase filter placed at bus $i$. Hence,

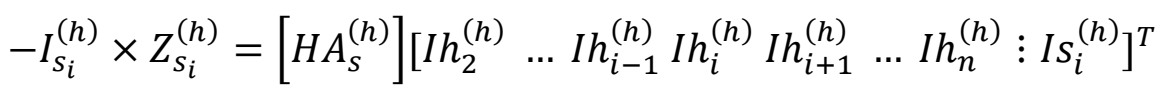

It is to be noted that in vector $\left[H A_{s}^{(h)}\right]$ the elements of $(n-1)$ columns are related to the harmonic currents of loads and the last element is related to the harmonic current of the filter placed at bus $i$. So it can be written as:

$$
\left[H A_{s}^{(h)}\right]=\left[H A_{s h}^{(h)} \vdots H A_{s S}^{(h)}\right]
$$

Substituting (4.51) into (4.50):

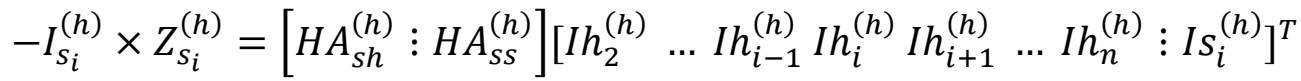

$$
\begin{aligned}
& -I_{s_{i}}^{(h)} \times Z_{s_{i}}^{(h)}=\left[\begin{array}{lllll}
H A_{s h}^{(h)}
\end{array}\right]\left[\begin{array}{lllll}
I h_{2}^{(h)} & \ldots & I h_{i-1}^{(h)} I h_{i}^{(h)} I h_{i+1}^{(h)} & \ldots & I h_{n}^{(h)}
\end{array}\right]^{T}+\left[\begin{array}{lll}
H A_{S S}^{(h)}
\end{array}\right] \\
& \times I s_{i}^{(h)} \\
& \left(\left[H A_{s s}^{(h)}\right]+Z_{s_{i}}^{(h)}\right) I s_{i}^{(h)}=\left[\begin{array}{lllll}
H A_{s h}^{(h)}
\end{array}\right]\left[\begin{array}{lllll}
I h_{2}^{(h)} & \ldots & I h_{i-1}^{(h)} I h_{i}^{(h)} I h_{i+1}^{(h)} & \ldots I h_{n}^{(h)}
\end{array}\right]^{T}
\end{aligned}
$$


In order to solve Equation 4.54 to get $I s_{i}^{(h)}$, the inverse of $\left(\left[H A_{s S}^{(h)}\right]+Z_{s_{i}}^{(h)}\right)$ is required:

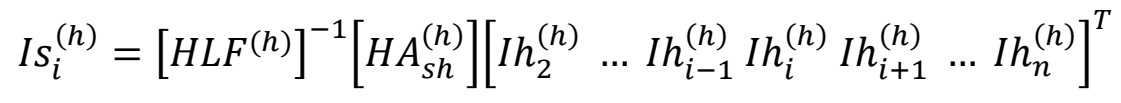

where,

$\left[H L F^{(h)}\right]=\left(\left[H A_{S S}^{(h)}\right]+Z_{s_{i}}^{(h)}\right)$

If $n l$ filters are installed in $\mathrm{RDS},\left[H L F^{(h)}\right]$ will be a square matrix of $(n l \times n l)$ dimension.

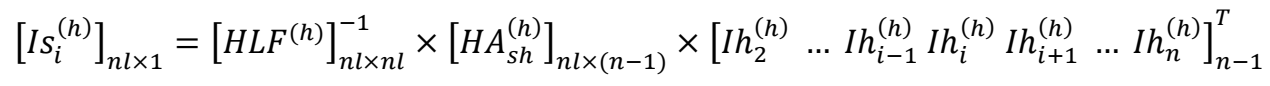

where,

$\left[I s_{i}^{(h)}\right]_{n l \times 1}=$ the harmonic current vector of the filters

Once the harmonic currents absorbed by the filters are obtained, the harmonic branch currents and bus voltages can also be known by using backward sweep as in Equation 4.31 and forward sweep as in Equation 4.46 respectively. The harmonic bus voltages are calculated iteratively until a predefined tolerance $\varepsilon$ is reached.

$$
\left|V_{i}^{(h), k+1}-V_{i}^{(h), k}\right| \leq \varepsilon
$$

where,

$V_{i}^{(h), k+1}=$ the harmonic voltage of bus $i$ for iteration $k+1$

$V_{i}^{(h), k}=$ the harmonic voltage of bus $i$ for iteration $k$

The total real power loss for $h^{\text {th }}$ harmonic is defined by:

$$
\operatorname{Ploss}^{(h)}=\sum_{i=1}^{b r} \operatorname{Ploss}_{i}^{(h)}=\sum_{i=1}^{b r} \sum_{h=h_{0}}^{h_{\max }}\left|B_{i}^{(h)}\right|^{2} \cdot R_{i}^{(h)}
$$


where,

$h_{0}=$ the smallest harmonic order

$h_{\max }=$ the highest harmonic order

$\left|B_{i}^{(h)}\right|=$ the magnitude of the ith branch current for the $h^{\text {th }}$ harmonic order

$R_{i}^{(h)}=$ the ith branch resistance for the $h^{\text {th }}$ harmonic order.

The total harmonic real power loss can also be expressed in terms of harmonic current vector as follows:

$$
\operatorname{PlosS}^{(h)}=\left[R^{(h)}\right]^{T} \cdot\left|\left[A^{(h)}\right]\left[I^{(h)}\right]\right|^{2}
$$

The root mean square values of the bus voltages $\left(V_{r m s_{i}}\right)$ can be known from the harmonic bus voltages calculated for all harmonic frequencies by utilizing the process described above:

$$
V_{r m s_{i}}=\sqrt{\left|V_{i}^{(1)}\right|^{2}+\sum_{h=h_{9}}^{h_{\max }}\left|V_{i}^{(h)}\right|^{2}}
$$

where,

$\left|V_{i}^{(1)}\right|=$ the magnitude of bus voltage at fundamental frequency

Finally, the total harmonic distortion at bus i can be calculated from the following equation:

$$
T H D_{i}(\%)=\frac{\sqrt{\sum_{h=h_{9}}^{h_{\max }}\left|V_{i}^{(h)}\right|^{2}}}{\left|V_{i}^{(1)}\right|^{2}}
$$




\section{Chapter 5: Results and Discussion}

Three test systems are selected to test the sizing and placement of harmonic filter problem through ant colony algorithm. At first, some harmonic current sources are introduced in the system and a solution of harmonic load flow through backward/forward sweep method is presented. After that the ant colony algorithm is used to determine the size and position of low pass harmonic filters. A total of nine different sizes (EATON Corporation Canada [45]) are made available to be selected and placed.

The three test systems used are Radial Distribution Systems (RDS). Bus 1 in each is a substation which provides the generated power to all distribution buses connected. In power systems the operation is only possible when total generated power $(\mathrm{Sg})$ is equal to total power demand $(\mathrm{Sd})$ :

$$
\begin{gathered}
S g-S d=0 \\
S g=P g+j Q g \\
S d=P d+j Q d
\end{gathered}
$$

The demand power is the sum of power consumed by the load (Sl) and total network losses incurred (Sloss):

$$
\mathrm{Sd}=\mathrm{Sl}+\text { Sloss }
$$

Hence we may write by substituting: 


$$
\mathrm{Sg}=\mathrm{Sl}+\text { Sloss }
$$

The total real power will be:

$$
\mathrm{Pg}=\mathrm{Pl}+\text { Ploss }
$$

The total power losses depend on the current injection of each individual bus and the impedance of transmission lines. The backward-forward sweep method described in Chapter 4 is used to calculate current injections and real power loss.

Moving forward, first harmonic current sources will be introduced at different locations of the system to demonstrate the effect of harmonics. These sources increase the total current injection of the buses they are connected to and thus result in increased losses. The amplitude and angle of harmonic buses is given by:

$$
\begin{gathered}
I(h)=\frac{1}{h \times \sin (h \omega t)} \\
\omega=2 \pi f
\end{gathered}
$$

where,

$f=$ frequency of the system

$h=$ harmonic order

The harmonics sources of $3^{\text {rd }}, 5^{\text {th }}, 7^{\text {th }}, 9^{\text {th }}, 11^{\text {th }}, 13^{\text {th }}$ and $15^{\text {th }}$ order are inserted. Moreover, backward/forward sweep method, as discussed in Chapter 4 is utilized to calculate the harmonic load flow solution. From harmonic load flow solution the total power losses due to fundamental frequency as well as harmonics and the total harmonic 
distribution are calculated. After getting the solution the ant colony system is utilized, which is explained in the next section.

\subsection{Implementation of Ant Colony System Algorithm}

In order to implement ant colony algorithm the first step is to create a matrix of all the available filter sizes and all the possible bus locations. Each available filter size is considered as a stage $\mathrm{i}(\mathrm{i}=1,2 \ldots \mathrm{n})$ and bus location as states $\mathrm{j}(\mathrm{j}=1,2 \ldots \mathrm{m})$. Here, $\mathrm{n}$ and $\mathrm{m}$ are the filter sizes and possible bus locations. Then a suitable number of ants are selected. The total numbers of ants selected in this case are half the total number of possible bus locations. Each ant starts its tour by selecting a randomly chosen state of first stage. Each ant selects its first stage randomly and tours around all the available states. The ACS algorithm is implemented as follows:

First all states of stages receive a certain and equal amount of pheromone. Then, $\mathrm{M}$ ants start their tour depending on the pheromone level, heuristic information and a probability function. After touring around all states, the pheromone of all states is locally updated. Furthermore, the value of the objective function is calculated for all ants. This objective function is the total real power loss. The state which results in minimizing the objective function the most is selected as best solution and recorded. After that, a global update of pheromone levels of all the states passed by respective ant and the best ant is made. This process is repeated until the maximum iterations are reached. ACS is implemented as follows:

Step 1: (Initialization): Generate a matrix for the total number of available filter sizes as stages and the number of possible bus locations as states and select a suitable value for control parameters $(\alpha, \beta)$, coefficient of pheromone evaporation $(\rho)$, the number of ants, 
states and stages. Also generate initial pheromone $\tau_{i j}$ and heuristic information $\eta_{i j}$ for all states. $\eta_{i j}$ is set to be the reciprocal of filters to be placed so if a bigger filter size is selected by an ant the heuristic information is less attractive. This is done because larger size filters are more expensive.

$$
\eta_{i j}=\frac{1}{L_{i}}
$$

where,

$L_{i}=$ filter size selected

Step 2: (Selection of a state (bus)): Ants use heuristic information which is the objective function and pheromone trails to select optimal allocation filter. An ant with randomly selected filter $\mathrm{j}$ will select a node $i$ on the basis of the following rule:

$$
I=\left\{\begin{array}{l}
\arg \max _{m \in A L_{i}}\left(\left[\tau_{i j}\right]^{\alpha}\left[\eta_{i j}\right]^{\beta}\right) \text { if } L_{i} \leq Q_{i} \\
\text { Otherwise }
\end{array}\right.
$$

where,

$L_{i}=$ the filter size selected (it's also the capacity of reactive power filter absorbs)

$Q_{i}=$ the total real power injection by the load on bus $i$.

$A L_{i}=$ the set of available states(buses) for randomly selected stage (filter)

$i$ is selected according to the probability distribution as follows:

$$
p_{i j}=\left\{\begin{array}{l}
\frac{\left[\tau_{i j}\right]^{\alpha}\left[\eta_{i j}\right]^{\beta}}{\sum_{m \in A L_{i}}\left[\tau_{i j}\right]^{\alpha}\left[\eta_{i j}\right]^{\beta}} \text { if } i \in A L_{i} \\
0 \quad \text { Otherwise }
\end{array}\right.
$$


Step 3: (Local Updating): Update the pheromone of all states passed by ants in Step 2 using the following equation:

$$
\tau_{i j}^{n e w}=(1-\rho) \tau_{i j}^{o l d}+\Delta \tau_{i j}^{k}
$$

where,

$\rho,\{0 \leq \rho \leq 1\}=$ the evaporation coefficient so that $(1-\rho)$ represent the pheromone evaporation

$\Delta \tau_{i j}^{k}=$ the contributions of ant $\mathrm{k}$ that used filter $\mathrm{j}$ and selected bus $\mathrm{i}$ to construct its solution

$\Delta \tau_{i j}^{k}$ is basically the amount of trail laid on edge (ij) by ant $\mathrm{k}$ which is calculated by:

$$
\Delta \tau_{i j}^{k}=\left\{\begin{array}{rr}
\frac{1}{O F^{k}} \text { if ant used placed filter } j \text { on bus } i \\
0 & \text { Otherwise }
\end{array}\right.
$$

where,

$O F^{k}=$ objective function of ant $\mathrm{k}$ (total real power loss)

The objective function is total real power loss so if filter $\mathrm{j}$ placed in bus i results in small power loss it will mean that the pheromone level of that particular ant will be bigger.

Step 4: (Objective Function Calculation): Calculate the objective function for all ants. The objective function is the total real power loss. Here the Radial Power Flow (RPF) and Harmonic Power Flow (HPF) algorithms are used to calculate total real power loss. The RPF calculates real power loss due to fundamental frequency and HPF calculates the real power loss due to harmonics. So the total power loss is given as follows:

$$
O F^{k}=\text { Ploss }=\text { Ploss }^{(1)}+\sum_{h=h_{0}}^{h_{\max }} \operatorname{Ploss}_{i}^{(h)}
$$


Step 5: (Global Updating): Update the pheromone of all states passed by ant which gave the best objective function using following equation:

$$
\tau_{i j}^{\text {new }}=(1-\rho) \tau_{i j}^{o l d}+\Delta \tau_{i j}^{\text {best }}
$$

where,

$\Delta \tau_{i j}^{b e s t}=$ the contributions of ant which used filter $\mathrm{j}$ and selected bus $\mathrm{i}$ to and resulted in best objective function

$$
\begin{gathered}
\Delta \tau_{i j}^{\text {best }}=\frac{1}{O F^{\text {best }}} \\
O F^{\text {best }}=\min _{1 \leq k \leq k_{\max }}\left(O F^{k}\right)
\end{gathered}
$$

where,

$O F^{\text {best }}=$ best objective function achieved

$k_{\max }=$ maximum number of ants

Step 6: (Convergence Criterion Checking): If total number of iterations reaches a maximum, the program is terminated and the results are presented, otherwise, increase iteration by 1 and go to step 2 and the whole process is repeated. 


\subsection{5-Bus Radial Distribution Test System}

The first test system is the 5-bus radial distribution system. The single line diagram of connection between buses is shown below:

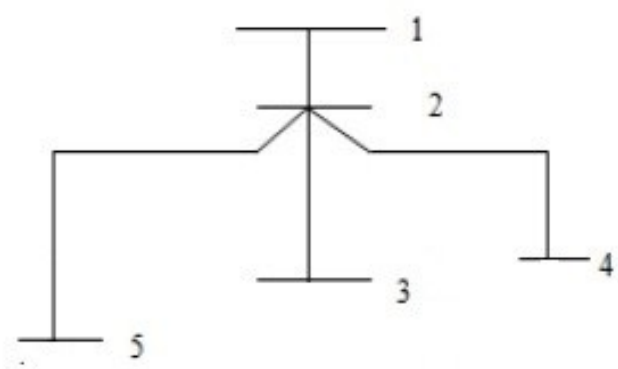

Figure 15: 5-bus radial distribution system

Bus 1 is the generation bus. All the remaining buses are load buses and distribute generated power to the loads connected to each bus. The total real power generated (Pg) is $\mathrm{Kw}$ and the total reactive power $(\mathrm{Qg})$ of the system is Kvar.

The following list provides a summary of the system bus and line data.

Table 3: 5-bus system bus and line data

\begin{tabular}{|c|c|c|c|c|}
\hline \multicolumn{5}{|c|}{ 5-bus system } \\
\hline \multirow{2}{*}{ Bus } & \multicolumn{2}{|c|}{ Generation } & \multicolumn{2}{c|}{ Demand } \\
\cline { 2 - 4 } & $\mathbf{P g}$ & $\mathbf{Q g}$ & $\mathbf{P d}$ & $\mathbf{Q d}$ \\
\hline 1 & 220 & 920 & 0 & 0 \\
\hline 2 & 0 & 0 & 100 & 30 \\
\hline 3 & 0 & 0 & 20 & 730 \\
\hline 4 & 0 & 0 & 50 & 150 \\
\hline 5 & 0 & 0 & 50 & 10 \\
\hline \multicolumn{5}{|c|}{ Line Data } \\
\hline From & To & $\mathbf{R}(\Omega)$ & $\mathbf{X}(\Omega)$ & \multirow{2}{*}{} \\
\hline 1 & 2 & 0.117 & 0.048 & \multirow{2}{*}{} \\
\hline 2 & 3 & 0.107 & 0.044 & \multicolumn{1}{|}{} \\
\hline 2 & 4 & 0.314 & 0.054 & \\
\hline 2 & 5 & 0.15 & 0.042 \\
\cline { 2 - 3 }
\end{tabular}

Total demand is distributed among 4 load buses respectively. The harmonic sources are placed at buses 3,4 and 5 . 


\subsubsection{Harmonic Load Flow Results Before Filter Placement}

The harmonic load flow solution is presented as follows:

Table 4: Harmonic Load flow results for 5-bus

\begin{tabular}{|c|c|c|c|c|c|c|c|c|c|c|}
\hline \multicolumn{11}{|c|}{ Results of Harmonic Load Flow } \\
\hline Bus No. & $|\mathbf{f}|$ & |v $3 \mathrm{hl}$ & |V $5 \mathrm{hl}$ & $\mid v 7 \mathrm{hl}$ & |v $9 \mathrm{hl}$ & $|\mathrm{V} 11 \mathrm{~h}|$ & $\mid \mathrm{V} 13 \mathrm{hl}$ & $|\mathrm{V} 15 \mathrm{~h}|$ & $\underline{\text { Vrms }}$ & $\underline{\mathrm{THD}(\%)}$ \\
\hline 1 & 1 & 0 & 0 & 0 & 0 & 0 & 0 & 0 & 0 & 0 \\
\hline 2 & 0.9959 & 0.0032 & 0.0028 & 0.0027 & 0.0026 & 0.0026 & 0.0026 & 0.0025 & 0.9959 & 0.7208 \\
\hline 3 & 0.9939 & 0.0062 & 0.0053 & 0.0051 & 0.0050 & 0.0049 & 0.0049 & 0.0049 & 0.9940 & 1.3841 \\
\hline 4 & 0.9945 & 0.0032 & 0.0028 & 0.0027 & 0.0026 & 0.0026 & 0.0026 & 0.0025 & 0.9945 & 0.7218 \\
\hline 5 & 0.9954 & 0.0032 & 0.0028 & 0.0027 & 0.0026 & 0.0026 & 0.0026 & 0.0025 & 0.9955 & 0.7211 \\
\hline
\end{tabular}

\begin{tabular}{|c|c|c|c|}
\hline $\mathrm{Pg}(\mathrm{Kw})$ & Qg(Kvar) & $\mathrm{Pl}(\mathrm{Kw})$ & Ql(Kvar) \\
\hline 220 & 920 & 216.14 & 914.88 \\
\hline \multicolumn{4}{|c|}{ Ploss (Kw) } \\
\hline \multicolumn{2}{|c|}{ Ploss_fundamental } & 1.72 & \multirow{2}{*}{3.861} \\
\hline Ploss_har & & 2.14 & \\
\hline \multicolumn{4}{|c|}{ Qloss (Kvar) } \\
\hline \multicolumn{2}{|c|}{ Qloss_fundamental } & 0.69 & \multirow{2}{*}{5.12} \\
\hline \multicolumn{2}{|c|}{ Qloss_harmonics } & 4.44 & \\
\hline
\end{tabular}

Real power losses due to fundamental frequency are $1.72 \mathrm{KW}$, losses caused due harmonics are $2.14 \mathrm{KW}$. Hence, the total real power loss is $3.861 \mathrm{KW}$.

\subsubsection{Harmonic Load Flow Results Before Filter Placement}

Now, ACS is implemented to find the best solution to minimize real power losses.

Total number of ants selected is 2 as there are 4 load buses. ACS results are as follows:

Table 5: Ant Colony results for 5-bus system

\begin{tabular}{|l|c|l|c|}
\hline \multicolumn{3}{|c|}{ Ant Colony Result } \\
\hline \multirow{2}{*}{ Best Location } & Bus & \multirow{2}{*}{ Best Filter Size } & Kvar \\
\cline { 4 - 4 } & 3 & & 600 \\
\hline
\end{tabular}




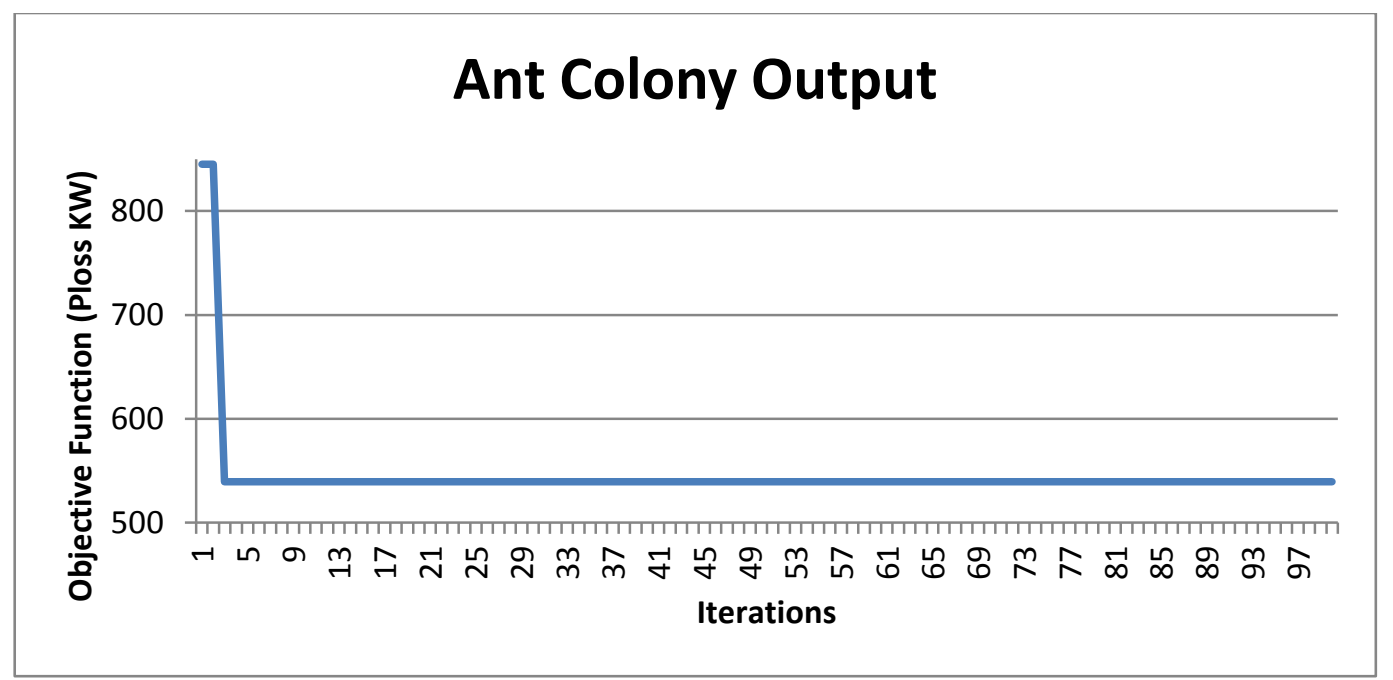

Figure 16: Ant colony output for 5-bus

The filter size selected is $600 \mathrm{Kvar}$ and the best location found to place it is bus 3 . The maximum number of iterations selected is 100 . ACS for 5-bus test system converged in 4 iterations. The harmonic load flow solution after placing this filter is given as follows:

Table 6: Harmonic Load flow results for 5-bus after filter placement

\begin{tabular}{|c|c|c|c|c|c|c|c|c|c|c|}
\hline \multicolumn{11}{|c|}{ Results of Harmonic Load Flow } \\
\hline Bus No. & $|v \mathbf{f}|$ & $|v 3 h|$ & |V $5 \mathrm{hl}$ & $\mid \mathrm{V} 7 \mathrm{hl}$ & |V $9 \mathrm{hl}$ & $|\mathrm{V} 11 \mathrm{~h}|$ & $|\mathrm{V} 13 \mathrm{~h}|$ & $|\mathrm{V} 15 \mathrm{~h}|$ & Vrms & $\underline{\mathrm{THD}(\%)}$ \\
\hline 1 & 1 & 0 & 0 & 0 & 0 & 0 & 0 & 0 & 0 & 0 \\
\hline 2 & 0.9976 & 0.0011 & 0.0010 & 0.0009 & 0.0009 & 0.0009 & 0.0009 & 0.0009 & 0.9976 & 0.2508 \\
\hline 3 & 0.9972 & 0.0022 & 0.0019 & 0.0018 & 0.0017 & 0.0017 & 0.0017 & 0.0017 & 0.9972 & 0.4808 \\
\hline 4 & 0.9962 & 0.0011 & 0.0010 & 0.0009 & 0.0009 & 0.0009 & 0.0009 & 0.0009 & 0.9962 & 0.2511 \\
\hline 5 & 0.9972 & 0.0011 & 0.0010 & 0.0009 & 0.0009 & 0.0009 & 0.0009 & 0.0009 & 0.9972 & 0.2509 \\
\hline
\end{tabular}

\begin{tabular}{|c|c|c|c|}
\hline Pg(Kw) & Qg(Kvar) & Pl(Kw) & Ql(Kvar) \\
\hline 220 & 920 & 219.46 & \multirow{2}{*}{919.36} \\
\hline \multicolumn{4}{|c|}{ Ploss (Kw) } \\
\hline Ploss_fundamental & 0.2793 & \multirow{2}{*}{0.539} \\
\cline { 1 - 2 } Ploss_harmonics & 0.2600 & \\
\cline { 1 - 2 } & Qloss (Kvar) \\
\hline Qloss_fundamental & 0.0952 & \multirow{2}{*}{0.634} \\
\hline Qloss_harmonics & 0.5388 & \\
\hline
\end{tabular}




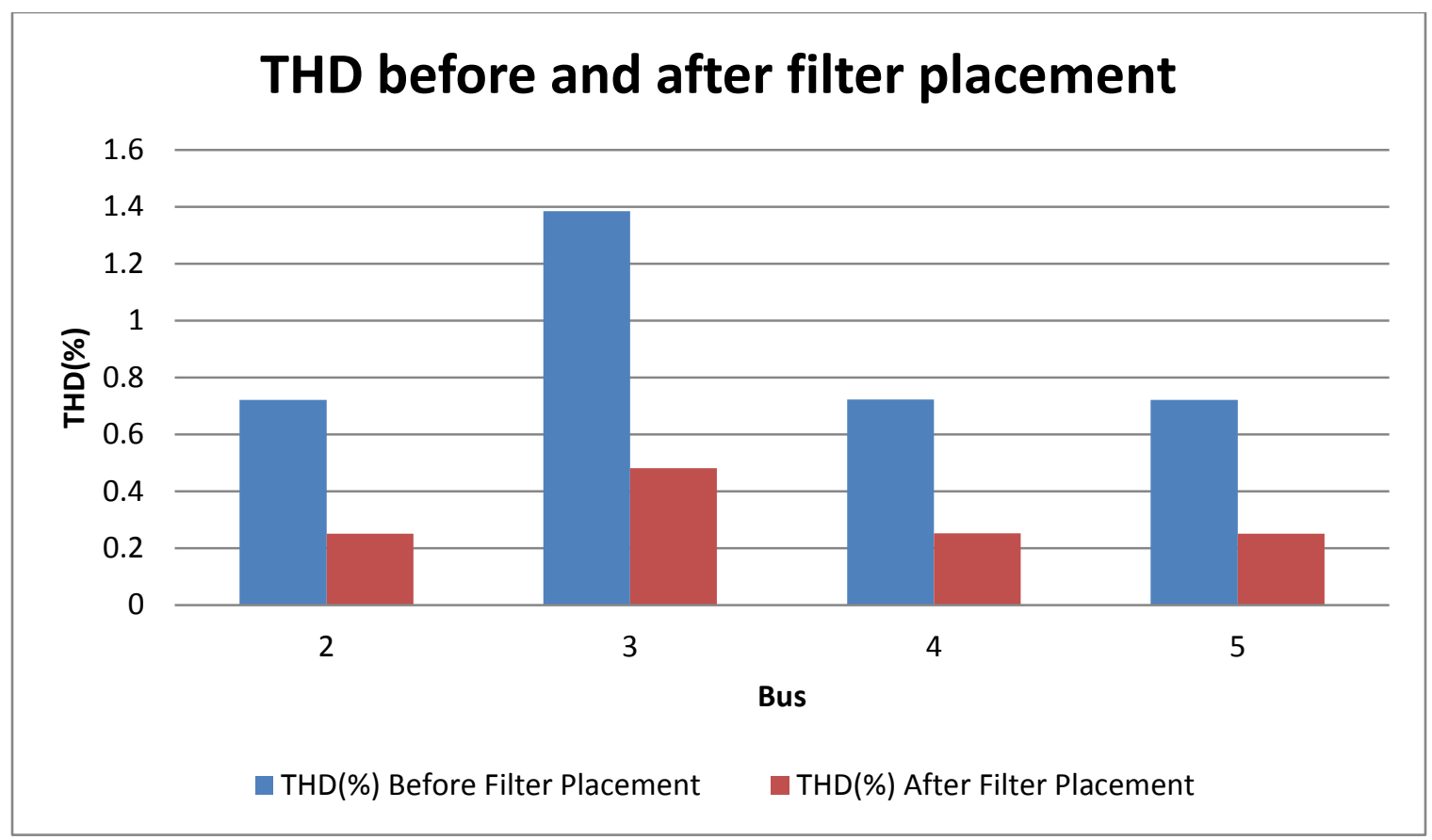

Figure 17: THD before and after filter placement for 5-bus system

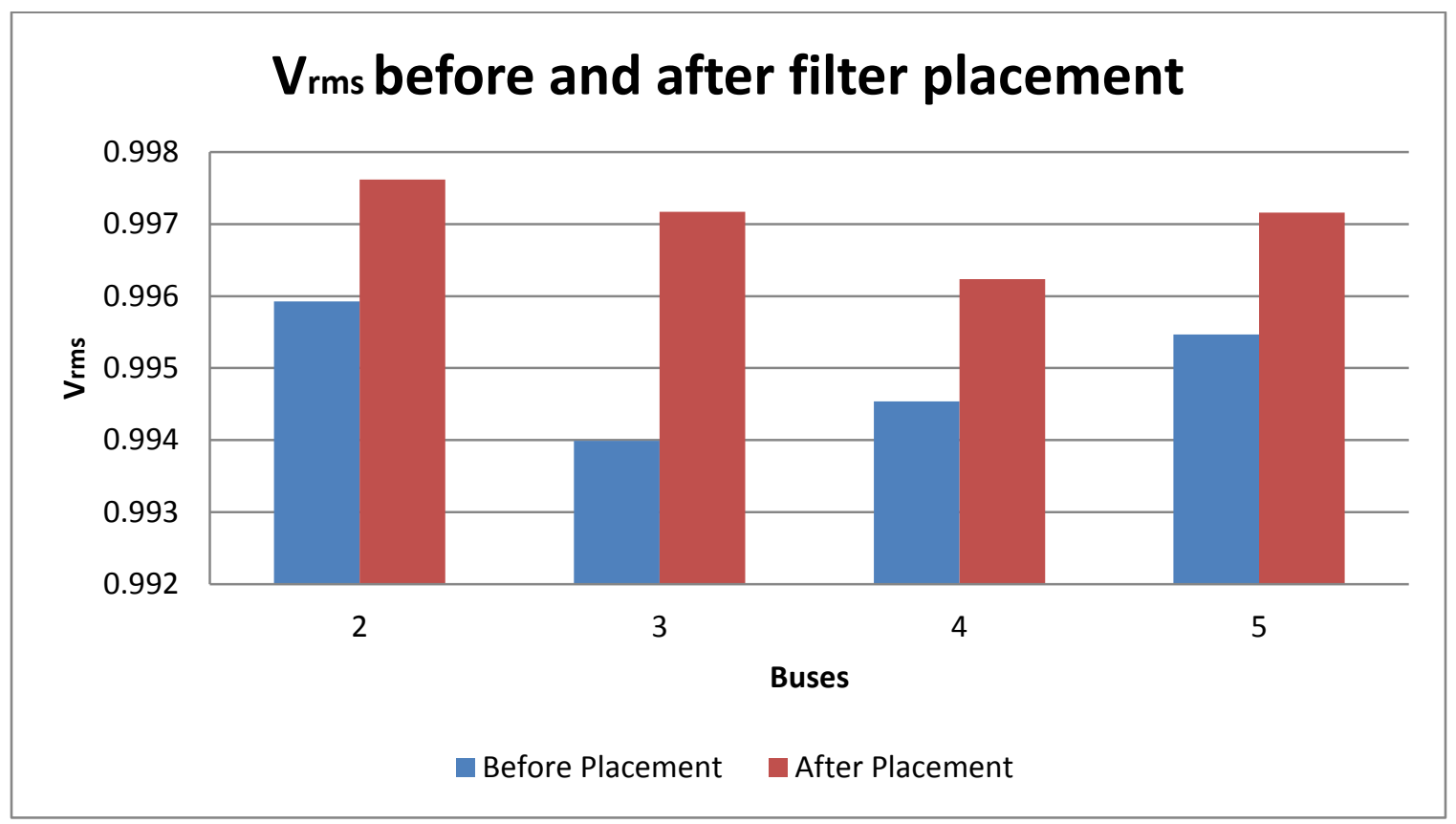

Figure 18: Vrms before and after filter placement for 5-bus system 
It can be seen that after placing the filter, the THD is suppressed as demonstrated in Figure 17. This has resulted in an improved voltage profile presented in Figure 18. The following table and graph demonstrate the effect of placing filter on power losses at fundamental frequency and harmonic of each order:

Table 7: Harmonic Load flow results for 5-bus after filter placement

\begin{tabular}{|l|c|c|c|}
\cline { 2 - 4 } \multicolumn{1}{c|}{} & $\begin{array}{c}\text { Before Filter } \\
\text { Placement }\end{array}$ & $\begin{array}{c}\text { After Filter } \\
\text { Placement }\end{array}$ & $\begin{array}{c}\text { \% } \\
\text { improvement }\end{array}$ \\
\cline { 2 - 4 } \multicolumn{1}{c|}{ Ploss(Kw) } & Ploss(Kw) & \\
\hline Fundamental & 1.721 & 0.2793 & $83.77 \%$ \\
\hline 3rd harmonic & 1.174 & 0.1426 & $87.85 \%$ \\
\hline 5th harmonic & 0.423 & 0.0514 & $87.85 \%$ \\
\hline 7th harmonic & 0.216 & 0.0262 & $87.85 \%$ \\
\hline 9th harmonic & 0.130 & 0.0158 & $87.85 \%$ \\
\hline 11th harmonic & 0.087 & 0.0106 & $87.85 \%$ \\
\hline 13th harmonic & 0.063 & 0.0076 & $87.85 \%$ \\
\hline 15th harmonic & 0.047 & 0.0057 & $87.85 \%$ \\
\hline Total & $\mathbf{3 . 8 6 1}$ & $\mathbf{0 . 5 3 9}$ & $\mathbf{8 6 . 0 3 \%}$ \\
\hline
\end{tabular}

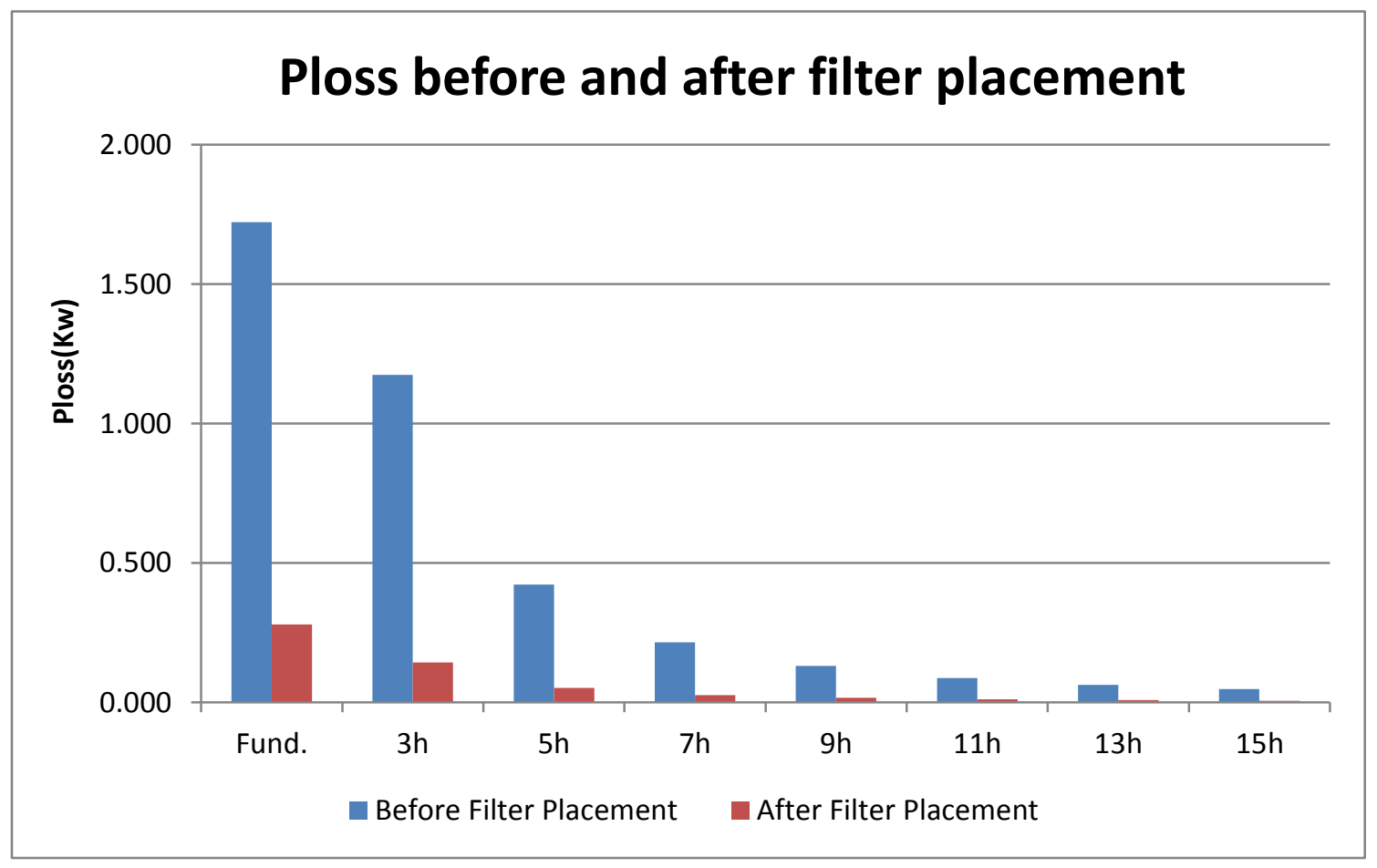

Figure 19: Ploss before and after filter placement for 5-bus system 
It can be seen that after placing the filter, the total power loss at each frequency is greatly reduced. Most of the power is lost $(1.721 \mathrm{KW})$ for the fundamental frequency which is impaired by $83.77 \%$ to $0.2793 \mathrm{KW}$ as a result of optimally placing the selected filter. Other significant losses of $1.174,0.423$ and $0.216 \mathrm{KW}$ each are incurred at $3^{\text {rd }}, 5^{\text {th }}$ and $7^{\text {th }}$ order harmonics respectively. These losses are also reduced to $0.1426,0.0514$ and $0.0262 \mathrm{KW}$ each. Almost $87.85 \%$ improvement is seen for harmonic losses. Also, the total power loss has been reduced from $3.861 \mathrm{KW}$ to $0.539 \mathrm{KW}$ giving an overall improvement of $86.03 \%$.

\subsection{1-Bus Radial Distribution Test System}

The first test system is the 11-bus radial distribution system. The single line diagram of connection between buses is shown below:

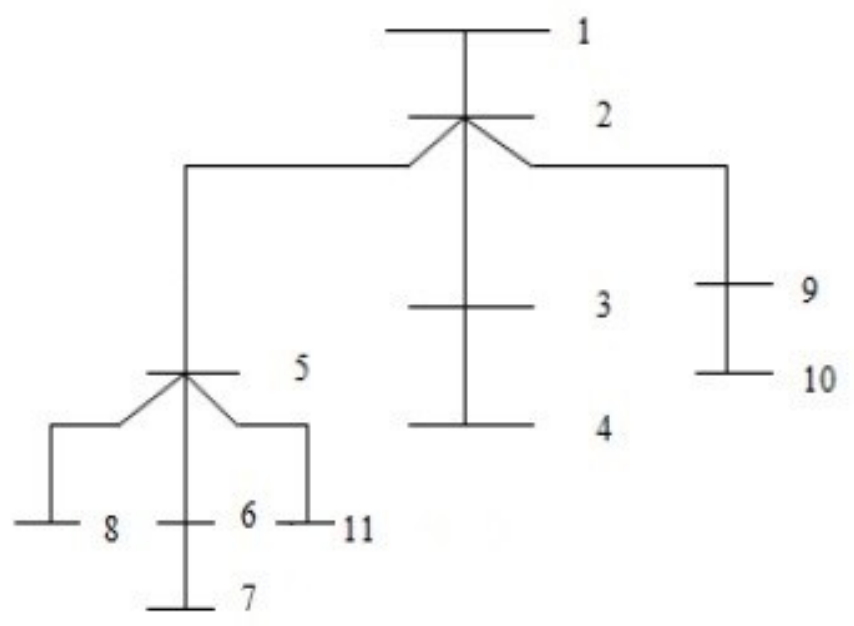

\section{Figure 20: 11-bus radial distribution system}

Bus 1 is the generation bus. All the remaining buses are load buses and distribute generated power to the loads connected to each bus. The total real power generated (Pg) 
is $\mathrm{Kw}$ and the total reactive power $(\mathrm{Qg})$ of the system is Kvar. The following list provides a summary of the system bus and line data.

Table 8: 11-bus system bus and line data

\begin{tabular}{|c|c|c|c|c|}
\hline \multicolumn{5}{|c|}{ 11-bus system } \\
\hline \multirow{2}{*}{ Bus } & \multicolumn{2}{|c|}{ Generation } & \multicolumn{2}{|c|}{ Demand } \\
\hline & $\mathrm{Pg}$ & Qg & Pd & Qd \\
\hline 1 & 940 & 4100 & 0 & 0 \\
\hline 2 & 0 & 0 & 100 & 30 \\
\hline 3 & 0 & 0 & 20 & 730 \\
\hline 4 & 0 & 0 & 150 & 225 \\
\hline 5 & 0 & 0 & 50 & 10 \\
\hline 6 & 0 & 0 & 120 & 540 \\
\hline 7 & 0 & 0 & 40 & 700 \\
\hline 8 & 0 & 0 & 75 & 90 \\
\hline 9 & 0 & 0 & 50 & 150 \\
\hline 10 & 0 & 0 & 125 & 825 \\
\hline 11 & 0 & 0 & 210 & 800 \\
\hline \multicolumn{4}{|c|}{ Line data } & \\
\hline From & To & $R(\Omega)$ & $X(\Omega)$ & \\
\hline 1 & 2 & 0.117 & 0.048 & \\
\hline 2 & 3 & 0.107 & 0.044 & \\
\hline 3 & 4 & 0.165 & 0.046 & \\
\hline 2 & 5 & 0.15 & 0.042 & \\
\hline 5 & 6 & 0.15 & 0.042 & \\
\hline 6 & 7 & 0.314 & 0.054 & \\
\hline 5 & 8 & 0.21 & 0.036 & \\
\hline 2 & 9 & 0.314 & 0.054 & \\
\hline 9 & 10 & 0.21 & 0.036 & \\
\hline 5 & 11 & 0.131 & 0.023 & \\
\hline
\end{tabular}

Total demand is distributed among 10 load buses. The harmonic sources are placed at buses 5, 7, 9, 10 and 11 . 


\subsubsection{Harmonic Load Flow Results Before Filter Placement}

The harmonic load flow solution is presented as follows:

Table 9: Harmonic Load flow results for 11-bus

\begin{tabular}{|c|c|c|c|c|c|c|c|c|c|c|}
\hline \multicolumn{11}{|c|}{ Results of Harmonic Load Flow } \\
\hline Bus No. & $\underline{\mid V} \mathbf{f} \mid$ & $\underline{\mid V} 3 \mathrm{hl}$ & IV $5 \mathrm{hl}$ & $\underline{\mid V} 7 \mathrm{hl}$ & |V $9 \mathrm{hl}$ & $|\mathrm{V} 11 \mathrm{~h}|$ & $|\mathrm{V} 13 \mathrm{~h}|$ & $\mid \mathrm{V} 15 \mathrm{hl}$ & $\underline{\text { Vrms }}$ & $\underline{\mathrm{THD}(\%)}$ \\
\hline 1 & 1.0000 & 0.0000 & 0.0000 & 0.0000 & 0 & 0 & 0 & 0 & 0 & 0 \\
\hline 2 & 0.9808 & 0.0094 & 0.0081 & 0.0077 & 0.0075 & 0.0074 & 0.0074 & 0.0074 & 0.9810 & 2.1195 \\
\hline 3 & 0.9772 & 0.0118 & 0.0102 & 0.0097 & 0.0095 & 0.0094 & 0.0093 & 0.0093 & 0.9775 & 2.6813 \\
\hline 4 & 0.9751 & 0.0118 & 0.0102 & 0.0097 & 0.0095 & 0.0094 & 0.0093 & 0.0093 & 0.9755 & 2.6870 \\
\hline 5 & 0.9706 & 0.0132 & 0.0111 & 0.0104 & 0.0102 & 0.0100 & 0.0099 & 0.0099 & 0.9710 & 2.9259 \\
\hline 6 & 0.9659 & 0.0170 & 0.0141 & 0.0132 & 0.0128 & 0.0126 & 0.0125 & 0.0124 & 0.9665 & 3.7304 \\
\hline 7 & 0.9627 & 0.0227 & 0.0181 & 0.0166 & 0.0160 & 0.0156 & 0.0154 & 0.0153 & 0.9638 & 4.7445 \\
\hline 8 & 0.9694 & 0.0132 & 0.0111 & 0.0104 & 0.0102 & 0.0100 & 0.0099 & 0.0099 & 0.9698 & 2.9293 \\
\hline 9 & 0.9741 & 0.0148 & 0.0119 & 0.0109 & 0.0105 & 0.0103 & 0.0102 & 0.0101 & 0.9745 & 3.0861 \\
\hline 10 & 0.9707 & 0.0185 & 0.0145 & 0.0132 & 0.0126 & 0.0123 & 0.0121 & 0.0120 & 0.9714 & 3.7542 \\
\hline 11 & 0.9678 & 0.0132 & 0.0111 & 0.0104 & 0.0102 & 0.0100 & 0.0099 & 0.0099 & 0.9682 & 2.9342 \\
\hline
\end{tabular}

\begin{tabular}{|c|c|c|c|}
\hline $\mathrm{Pg}(\mathrm{Kw})$ & Qg(Kvar) & $\mathrm{Pl}(\mathrm{Kw})$ & Ql(Kvar) \\
\hline 940 & 4100 & 878.07 & 4054.06 \\
\hline \multicolumn{4}{|c|}{ Ploss (Kw) } \\
\hline \multicolumn{2}{|c|}{ Ploss_fundamental } & 41.01 & \multirow{2}{*}{61.926} \\
\hline Ploss_har! & & 20.91 & \\
\hline \multicolumn{4}{|c|}{ Qloss (Kvar) } \\
\hline Qloss_fun & ental & 13.69 & \multirow{2}{*}{45.94} \\
\hline \multicolumn{2}{|c|}{ Qloss_harmonics } & 32.25 & \\
\hline
\end{tabular}

Real power losses due to fundamental frequency are $41.01 \mathrm{KW}$, the losses caused due harmonics are $20.91 \mathrm{KW}$. Hence, the total real power loss is $61.926 \mathrm{KW}$. 


\subsubsection{Harmonic Load Flow Results After Filter Placement}

Now, ACS is implemented to find the best solution to minimize real power losses.

Total number of ants selected is 5 as there are 10 load buses. ACS results are as follows:

Table 10: Ant Colony results for 11-bus system

\begin{tabular}{|l|c|l|c|}
\hline \multicolumn{3}{|c|}{ Ant Colony Result } \\
\hline \multirow{2}{*}{ Best Location } & Bus & \multirow{2}{*}{ Best Filter Size } & Kvar \\
\cline { 2 - 2 } & 10 & & 600 \\
\hline
\end{tabular}

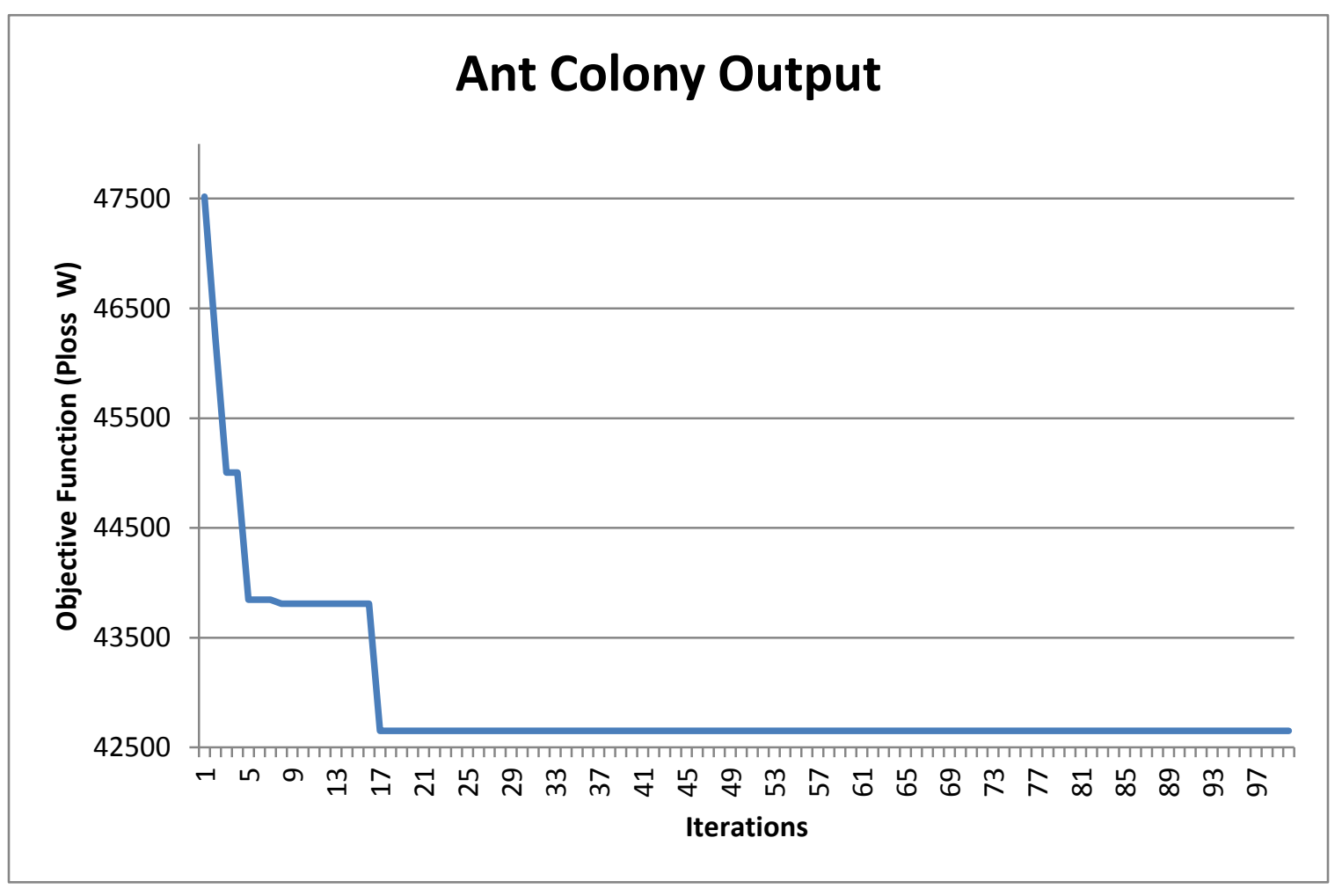

Figure 21: Ant colony output for 11-bus

The filter size selected is $600 \mathrm{Kvar}$ and the best location found to place it is bus 10. The maximum number of iterations selected is 100. ACS for 11-bus test system converged in 18 iterations. The harmonic load flow solution after placing this filter is given as follows: 
Table 11: Harmonic load flow results for 11-bus after filter placement

\begin{tabular}{|c|c|c|c|c|c|c|c|c|c|c|}
\hline \multicolumn{11}{|c|}{ Results after placement and sizing of filter by Ant Colony Algorithm } \\
\hline $\begin{array}{l}\text { Bus } \\
\text { No. }\end{array}$ & $|v f|$ & $\mid \mathrm{V} 3 \mathrm{hl}$ & $\mid \mathrm{V} 5 \mathrm{hl}$ & $|\mathrm{V} 7 \mathrm{~h}|$ & $\mid \mathrm{V} 9 \mathrm{hl}$ & $|\mathrm{V} 11 \mathrm{~h}|$ & $|\mathrm{V} 13 \mathrm{~h}|$ & $|\mathrm{V} 15 \mathrm{~h}|$ & $\underline{\text { Vrms }}$ & $\underline{\mathrm{THD}(\%)}$ \\
\hline 1 & 1.0000 & 0.0000 & 0.0000 & 0.0000 & 0 & 0 & 0 & 0 & 0 & 0 \\
\hline 2 & 0.9828 & 0.0072 & 0.0062 & 0.0059 & 0.0058 & 0.0057 & 0.0057 & 0.0056 & 0.9829 & 1.6239 \\
\hline 3 & 0.9792 & 0.0096 & 0.0083 & 0.0079 & 0.0077 & 0.0076 & 0.0076 & 0.0076 & 0.9794 & 2.1797 \\
\hline 4 & 0.9771 & 0.0096 & 0.0083 & 0.0079 & 0.0077 & 0.0076 & 0.0076 & 0.0076 & 0.9774 & 2.1844 \\
\hline 5 & 0.9726 & 0.0087 & 0.0074 & 0.0070 & 0.0068 & 0.0068 & 0.0067 & 0.0067 & 0.9728 & 1.9548 \\
\hline 6 & 0.9679 & 0.0102 & 0.0086 & 0.0081 & 0.0079 & 0.0078 & 0.0078 & 0.0077 & 0.9681 & 2.2835 \\
\hline 7 & 0.9648 & 0.0118 & 0.0097 & 0.0091 & 0.0088 & 0.0087 & 0.0086 & 0.0085 & 0.9651 & 2.5712 \\
\hline 8 & 0.9715 & 0.0087 & 0.0074 & 0.0070 & 0.0068 & 0.0068 & 0.0067 & 0.0067 & 0.9716 & 1.9571 \\
\hline 9 & 0.9783 & 0.0127 & 0.0101 & 0.0092 & 0.0088 & 0.0086 & 0.0085 & 0.0085 & 0.9786 & 2.5954 \\
\hline 10 & 0.9763 & 0.0165 & 0.0127 & 0.0115 & 0.0109 & 0.0106 & 0.0105 & 0.0103 & 0.9768 & 3.2621 \\
\hline 11 & 0.9698 & 0.0087 & 0.0074 & 0.0070 & 0.0068 & 0.0068 & 0.0067 & 0.0067 & 0.9700 & 1.9604 \\
\hline
\end{tabular}

\begin{tabular}{|c|c|c|c|}
\hline $\mathrm{Pg}(\mathrm{Kw})$ & Qg(Kvar) & $\mathrm{Pl}(\mathrm{Kw})$ & QI(Kvar) \\
\hline 940 & 4100 & 897.35 & 4071.54 \\
\hline \multicolumn{4}{|c|}{ Ploss (Kw) } \\
\hline \multicolumn{2}{|c|}{ Ploss_fundamental } & 31.09 & \multirow{2}{*}{42.65} \\
\hline Ploss_har & & 11.56 & \\
\hline \multicolumn{4}{|c|}{ Qloss (Kvar) } \\
\hline \multicolumn{2}{|c|}{ Qloss_fundamental } & 10.61 & \multirow{2}{*}{28.46} \\
\hline \multicolumn{2}{|c|}{ Qloss_harmonics } & 17.85 & \\
\hline
\end{tabular}




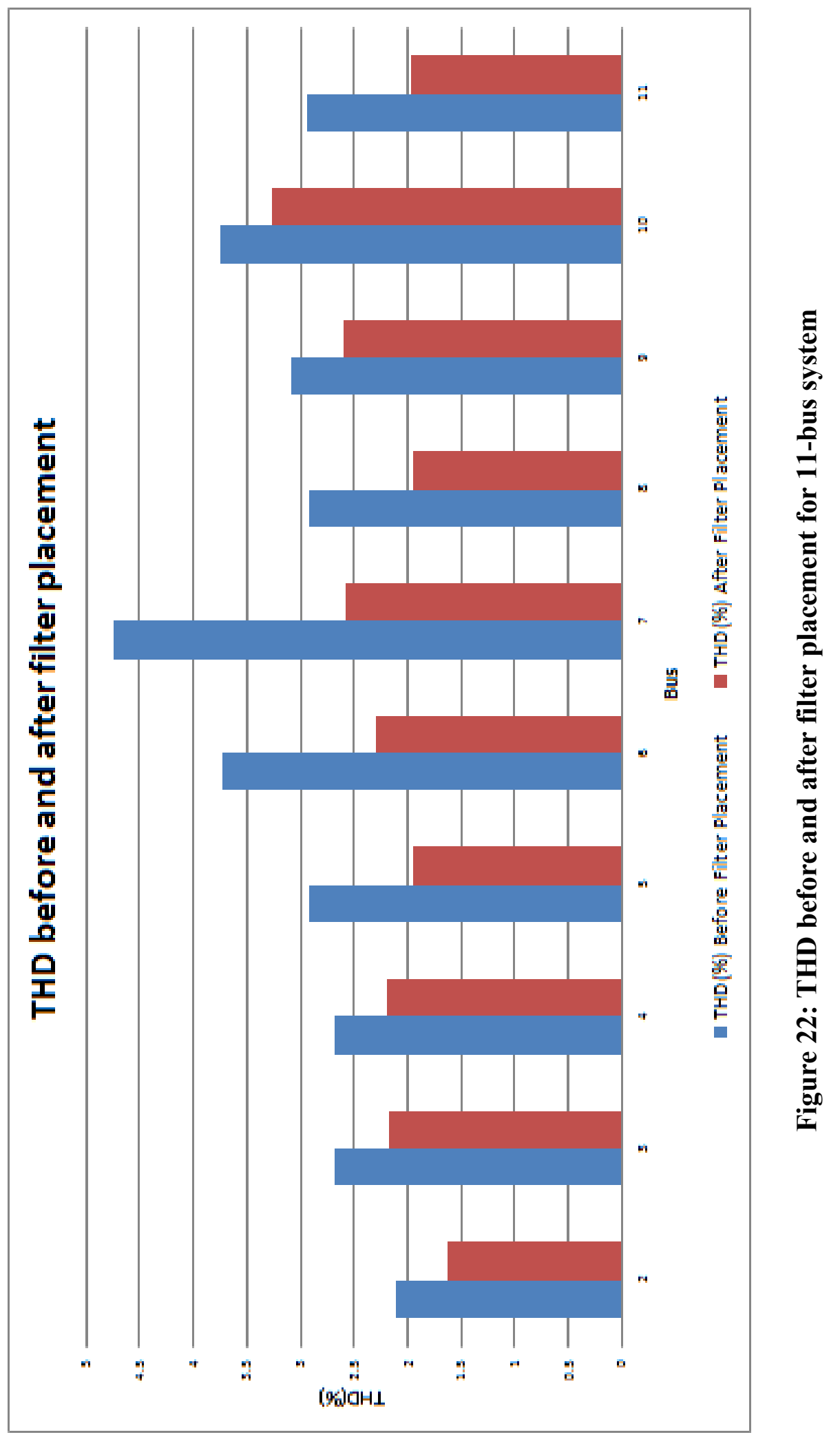




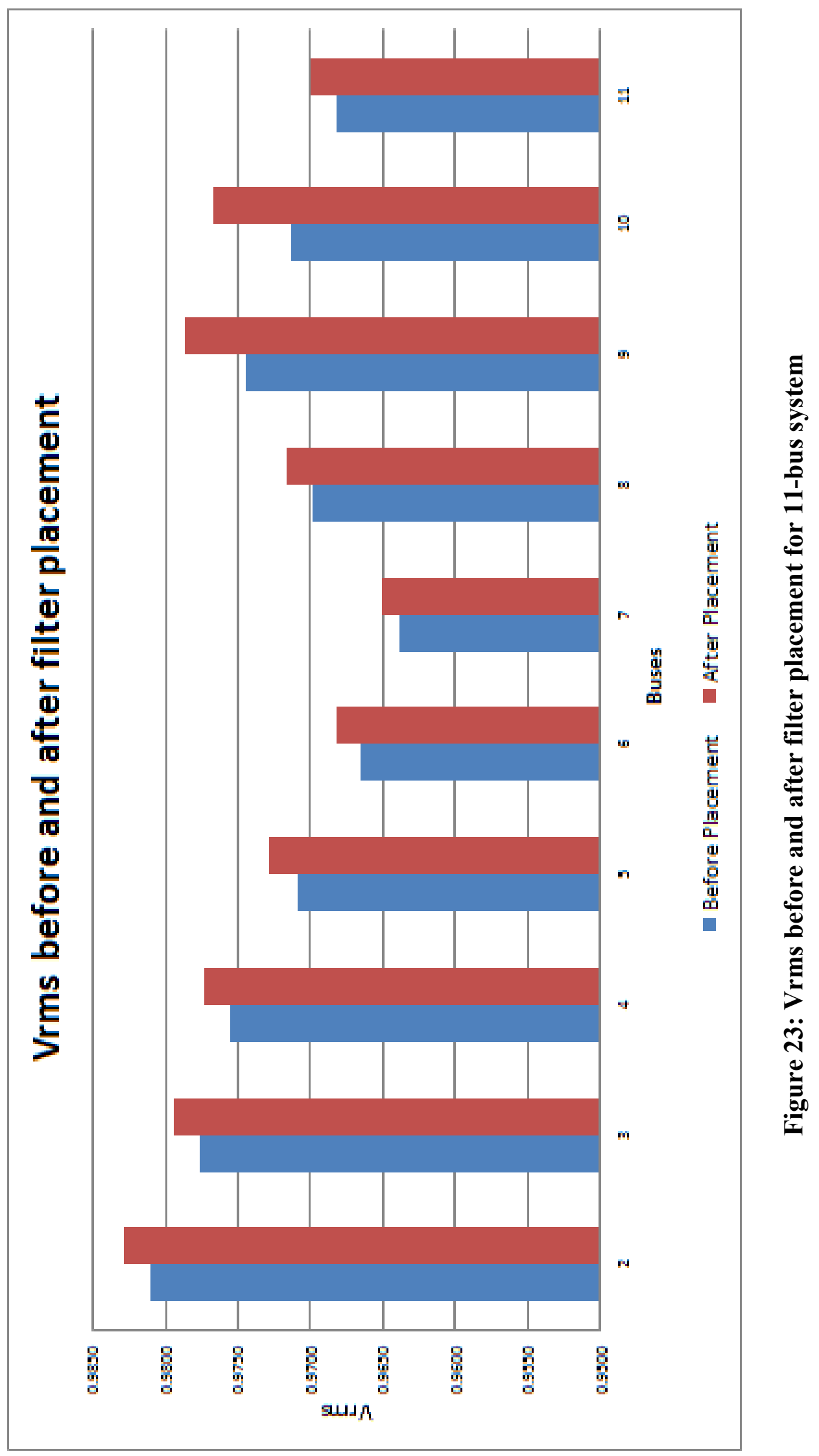


It can be seen that after placing the filter, the THD is suppressed as demonstrated in Figure 22. This has resulted in an improved voltage profile presented in Figure 23. The following table and graph demonstrate the effect of placing filter on power losses at fundamental frequency and harmonic of each order:

Table 12: Harmonic Load flow results for 11-bus after filter placement

\begin{tabular}{|l|c|c|c|}
\cline { 2 - 3 } \multicolumn{1}{c|}{} & $\begin{array}{c}\text { Before Filter } \\
\text { Placement }\end{array}$ & $\begin{array}{c}\text { After Filter } \\
\text { Placement }\end{array}$ & $\begin{array}{c}\text { \% } \\
\text { improvement }\end{array}$ \\
\cline { 2 - 3 } & Ploss(Kw) & Ploss(Kw) & \\
\hline Fundamental & 41.011 & 31.092 & $24.19 \%$ \\
\hline 3rd harmonic & 11.476 & 6.344 & $44.73 \%$ \\
\hline 5th harmonic & 4.132 & 2.284 & $44.73 \%$ \\
\hline 7th harmonic & 2.108 & 1.165 & $44.73 \%$ \\
\hline 9th harmonic & 1.275 & 0.705 & $44.73 \%$ \\
\hline 11th harmonic & 0.854 & 0.472 & $44.73 \%$ \\
\hline 13th harmonic & 0.611 & 0.338 & $44.73 \%$ \\
\hline 15th harmonic & 0.459 & 0.254 & $44.73 \%$ \\
\hline Total & $\mathbf{6 1 . 9 2 6}$ & $\mathbf{4 2 . 6 5}$ & $\mathbf{3 1 . 1 2 \%}$ \\
\hline
\end{tabular}

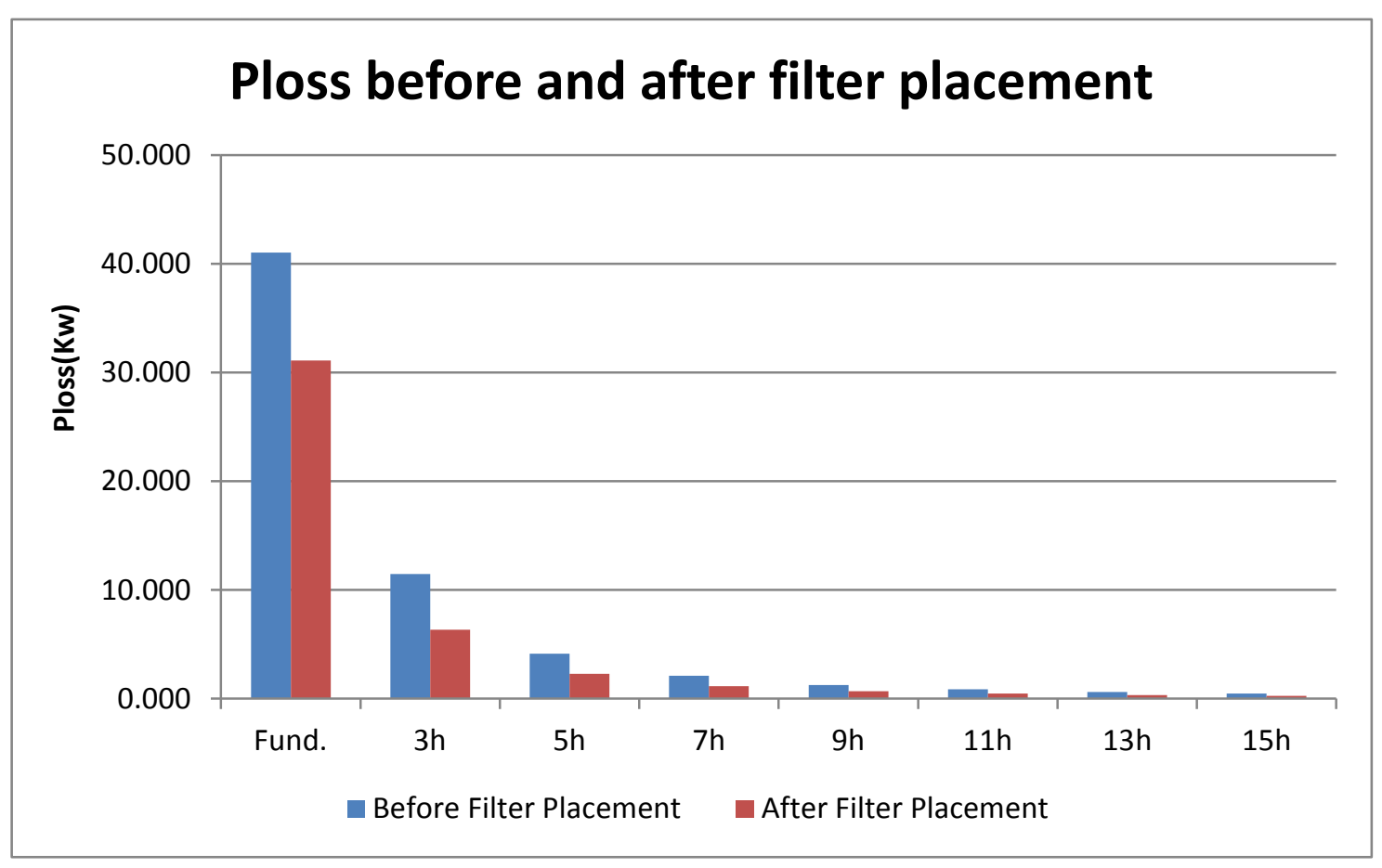

Figure 24: Ploss before and after filter placement for 11-bus system 
After placing the filter, the total power loss at each frequency is reduced. Most of the power is lost $(41.011 \mathrm{KW})$ for the fundamental frequency component which is improved by $24.19 \%$ to $31.092 \mathrm{KW}$. Other noticeable losses are $11.476,4.132,2.108$ and $1.275 \mathrm{KW}$ each at $3^{\text {rd }}, 5^{\text {th }}, 7^{\text {th }}$ and $9^{\text {th }}$ order harmonics respectively. These losses are reduced to $6.344,2.284,1.165$ and $0.705 \mathrm{KW}$ each. Almost $44.73 \%$ improvement is seen for harmonic losses. Also, the total power loss has been reduced from $61.926 \mathrm{KW}$ to $42.65 \mathrm{KW}$ giving an overall improvement of $31.12 \%$ and a saving of almost $19.27 \mathrm{KW}$.

\subsection{3-Bus Radial Distribution Test System}

The first test system is the 13-bus radial distribution system. The single line diagram of connection between buses is shown below:

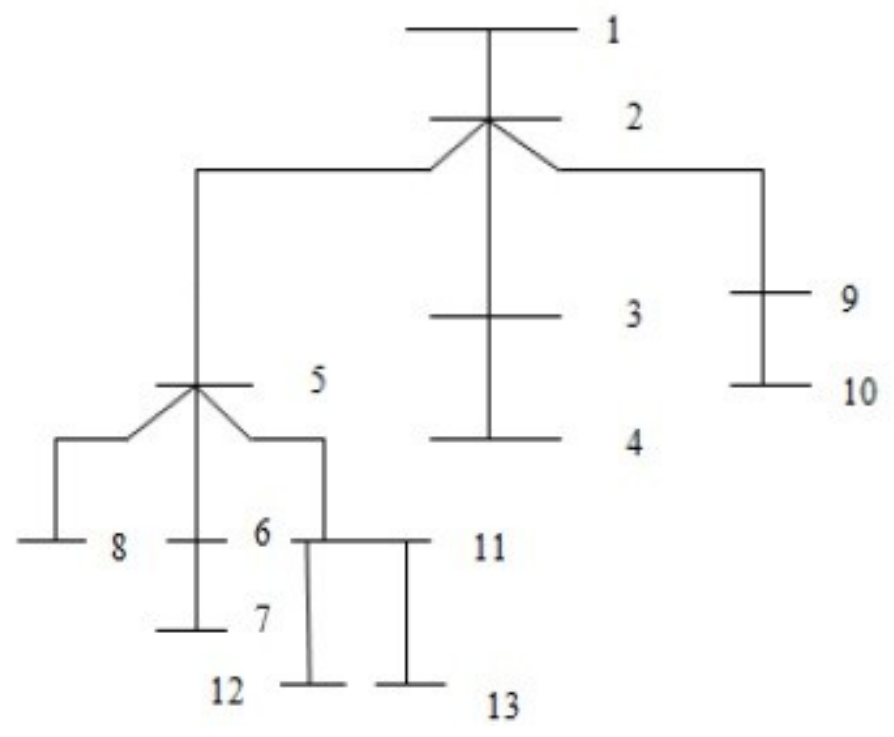

Figure 25: 13-bus radial distribution system

Bus 1 is the generation bus. All the remaining buses are load buses and distribute generated power to the loads connected to each bus. The total real power generated (Pg) is $\mathrm{Kw}$ and the total reactive power $(\mathrm{Qg})$ of the system is Kvar. The following list provides a summary of the system bus and line data. 
Table 13: 13-bus system bus and line data

\begin{tabular}{|c|c|c|c|c|}
\hline \multicolumn{5}{|c|}{ 13-bus data } \\
\hline \multirow{2}{*}{ Bus } & \multicolumn{2}{|c|}{ Generation } & \multicolumn{2}{|c|}{ Demand } \\
\hline & $\mathrm{Pg}$ & Qg & $\mathbf{P d}$ & Qd \\
\hline 1 & 1115 & 4430 & 0 & 0 \\
\hline 2 & 0 & 0 & 100 & 30 \\
\hline 3 & 0 & 0 & 20 & 730 \\
\hline 4 & 0 & 0 & 150 & 225 \\
\hline 5 & 0 & 0 & 50 & 10 \\
\hline 6 & 0 & 0 & 120 & 540 \\
\hline 7 & 0 & 0 & 40 & 700 \\
\hline 8 & 0 & 0 & 75 & 90 \\
\hline 9 & 0 & 0 & 50 & 150 \\
\hline 10 & 0 & 0 & 125 & 825 \\
\hline 11 & 0 & 0 & 210 & 800 \\
\hline 12 & 0 & 0 & 80 & 300 \\
\hline 13 & 0 & 0 & 95 & 30 \\
\hline \multicolumn{4}{|c|}{ Line Data } & \\
\hline From & To & $R(\Omega)$ & $x(\Omega)$ & \\
\hline 1 & 2 & 0.117 & 0.048 & \\
\hline 2 & 3 & 0.107 & 0.044 & \\
\hline 3 & 4 & 0.165 & 0.046 & \\
\hline 2 & 5 & 0.15 & 0.042 & \\
\hline 5 & 6 & 0.15 & 0.042 & \\
\hline 6 & 7 & 0.314 & 0.054 & \\
\hline 5 & 8 & 0.21 & 0.036 & \\
\hline 2 & 9 & 0.314 & 0.054 & \\
\hline 9 & 10 & 0.21 & 0.036 & \\
\hline 5 & 11 & 0.131 & 0.023 & \\
\hline 11 & 12 & 0.105 & 0.018 & \\
\hline 11 & 13 & 0.157 & 0.027 & \\
\hline
\end{tabular}

Total demand is distributed among 12 load buses respectively . The harmonic sources are placed at buses5, 7, 9, 10, 12 and 13 . 


\subsubsection{Harmonic Load Flow Results Before Filter Placement}

The harmonic load flow solution is presented as follows:

Table 14: Harmonic Load flow results for 13-bus

\begin{tabular}{|c|c|c|c|c|c|c|c|c|c|c|}
\hline \multicolumn{11}{|c|}{ Results of Harmonic Load Flow } \\
\hline Bus No. & $|v \mathrm{f}|$ & |V $3 \mathrm{hl}$ & IV $5 \mathrm{hl}$ & $\mid \mathrm{V} 7 \mathrm{hl}$ & IV 9hI & $|\mathrm{V} 11 \mathrm{~h}|$ & $|V \quad 13 \mathrm{~h}|$ & $|v \quad 15 \mathrm{~h}|$ & Vrms & $\underline{\mathrm{THD}(\%)}$ \\
\hline 1 & 1 & 0 & 0 & 0 & 0 & 0 & 0 & 0 & 0 & 0 \\
\hline 2 & 0.9784 & 0.0077 & 0.0066 & 0.0063 & 0.0062 & 0.0061 & 0.0060 & 0.0060 & 0.9785 & 1.74 \\
\hline 3 & 0.9748 & 0.0101 & 0.0087 & 0.0083 & 0.0081 & 0.0080 & 0.0080 & 0.0079 & 0.9750 & 2.30 \\
\hline 4 & 0.9727 & 0.0101 & 0.0087 & 0.0083 & 0.0081 & 0.0080 & 0.0080 & 0.0079 & 0.9729 & 2.31 \\
\hline 5 & 0.9656 & 0.0096 & 0.0082 & 0.0077 & 0.0075 & 0.0074 & 0.0074 & 0.0073 & 0.9658 & 2.17 \\
\hline 6 & 0.9609 & 0.0102 & 0.0086 & 0.0082 & 0.0080 & 0.0078 & 0.0078 & 0.0078 & 0.9611 & 2.31 \\
\hline 7 & 0.9577 & 0.0112 & 0.0094 & 0.0088 & 0.0085 & 0.0084 & 0.0083 & 0.0083 & 0.9580 & 2.50 \\
\hline 8 & 0.9644 & 0.0096 & 0.0082 & 0.0077 & 0.0075 & 0.0074 & 0.0074 & 0.0073 & 0.9647 & 2.17 \\
\hline 9 & 0.9716 & 0.0133 & 0.0106 & 0.0097 & 0.0093 & 0.0091 & 0.0090 & 0.0089 & 0.9720 & 2.75 \\
\hline 10 & 0.9682 & 0.0172 & 0.0133 & 0.0120 & 0.0114 & 0.0111 & 0.0109 & 0.0108 & 0.9688 & 3.43 \\
\hline 11 & 0.9609 & 0.0105 & 0.0088 & 0.0083 & 0.0081 & 0.0080 & 0.0079 & 0.0079 & 0.9612 & 2.35 \\
\hline 12 & 0.9601 & 0.0113 & 0.0094 & 0.0088 & 0.0085 & 0.0084 & 0.0083 & 0.0083 & 0.9604 & 2.49 \\
\hline 13 & 0.9600 & 0.0105 & 0.0088 & 0.0083 & 0.0081 & 0.0080 & 0.0079 & 0.0079 & 0.9603 & 2.35 \\
\hline
\end{tabular}

\begin{tabular}{|c|c|c|c|}
\hline $\mathrm{Pg}(\mathrm{Kw})$ & Qg(Kvar) & $\mathrm{PI}(\mathrm{Kw})$ & QI(Kvar) \\
\hline 1115 & 4430 & 1053.003178 & 4393.88 \\
\hline \multicolumn{4}{|c|}{ Ploss (Kw) } \\
\hline \multicolumn{2}{|c|}{ Ploss_fundamental } & 49.36 & \multirow{2}{*}{61.997} \\
\hline Ploss_har & & 12.63 & \\
\hline \multicolumn{4}{|c|}{ Qloss (Kvar) } \\
\hline \multicolumn{2}{|c|}{ Qloss_fundamental } & 16.45 & \multirow{2}{*}{36.12} \\
\hline \multicolumn{2}{|c|}{ Qloss_harmonics } & 19.68 & \\
\hline
\end{tabular}

Real power losses due to fundamental frequency are $49.36 \mathrm{KW}$, whereas the losses due the presence of harmonics are $12.63 \mathrm{KW}$. Hence, the total real power losses are $61.997 \mathrm{KW}$. 


\subsubsection{Harmonic Load Flow Results After Filter Placement}

Now, ACS is implemented to find the best solution to minimize real power losses.

Total number of ants selected is 6 as there are 12 load buses. ACS results are as follows:

Table 15: Ant Colony results for 13-bus system

\begin{tabular}{|l|c|c|c|}
\hline \multicolumn{4}{|c|}{ Ant Colony Result } \\
\hline \multirow{2}{*}{ Best Location } & Bus & \multirow{2}{*}{ Best Filter Size } & Kvar \\
\cline { 2 - 2 } & 7 & & 600 \\
\hline
\end{tabular}

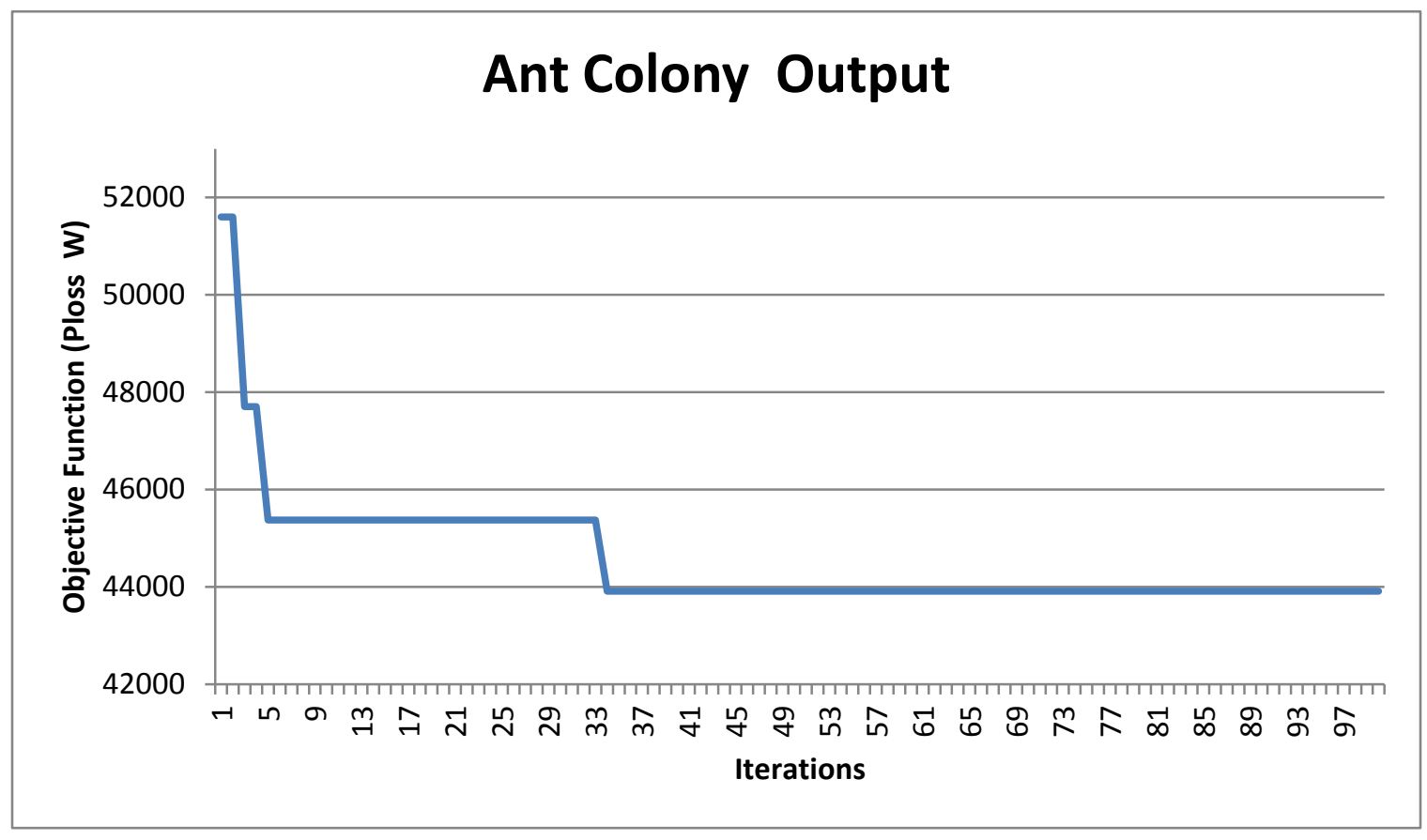

Figure 26: Ant colony output for 11-bus

The filter size selected is $600 \mathrm{Kvar}$ and the best location found to place it is bus 7 . It converged after 35 iterations and after that it is saturated. The maximum number of iterations selected is 100. ACS for 13-bus test system converged in 35 iterations. The harmonic load flow solution after placing this filter is given as follows: 
Table 16: Harmonic load flow results for 13-bus after filter placement

\begin{tabular}{|c|c|c|c|c|c|c|c|c|c|c|}
\hline \multicolumn{11}{|c|}{ Results after placement and sizing of filter by Ant Colony Algorithm } \\
\hline Bus No. & $\lfloor\mathbf{f l}$ & $\mid \mathrm{V} 3 \mathrm{hl}$ & $\lfloor\mathbf{V} 5 \mathrm{~h} \mid$ & $\lfloor\mathrm{V} 7 \mathrm{hl}$ & $\lfloor\mathrm{V} 9 \mathrm{~h}$ & $|\mathrm{V} 11 \mathrm{~h}|$ & $\lfloor\mathbf{V} 13 \mathrm{~h} \mid$ & $|\mathrm{V} 15 \mathrm{~h}|$ & $\underline{\text { Vrms }}$ & $\underline{\mathrm{THD}(\%)}$ \\
\hline 1 & 1.0000 & 0.0000 & 0.0000 & 0.0000 & 0.0000 & 0.0000 & 0.0000 & 0.0000 & 0.0000 & 0.0000 \\
\hline 2 & 0.9805 & 0.0055 & 0.0047 & 0.0045 & 0.0044 & 0.0043 & 0.0043 & 150.6753 & 0.9806 & 1.2399 \\
\hline 3 & 0.9770 & 0.0059 & 0.0051 & 0.0049 & 0.0048 & 0.0047 & 0.0047 & 148.9549 & 0.9771 & 1.3471 \\
\hline 4 & 0.9749 & 0.0059 & 0.0051 & 0.0049 & 0.0048 & 0.0047 & 0.0047 & 148.9548 & 0.9750 & 1.3500 \\
\hline 5 & 0.9696 & 0.0075 & 0.0063 & 0.0059 & 0.0058 & 0.0057 & 0.0057 & 147.8229 & 0.9697 & 1.6665 \\
\hline 6 & 0.9665 & 0.0081 & 0.0068 & 0.0064 & 0.0062 & 0.0061 & 0.0061 & 147.6890 & 0.9667 & 1.7975 \\
\hline 7 & 0.9655 & 0.0091 & 0.0075 & 0.0070 & 0.0068 & 0.0067 & 0.0066 & 146.8956 & 0.9657 & 1.9856 \\
\hline 8 & 0.9685 & 0.0075 & 0.0063 & 0.0059 & 0.0058 & 0.0057 & 0.0057 & 147.8229 & 0.9686 & 1.6685 \\
\hline 9 & 0.9738 & 0.0112 & 0.0088 & 0.0079 & 0.0076 & 0.0074 & 0.0073 & 149.3053 & 0.9741 & 2.2531 \\
\hline 10 & 0.9705 & 0.0151 & 0.0115 & 0.0103 & 0.0097 & 0.0094 & 0.0092 & 148.8602 & 0.9709 & 2.9420 \\
\hline 11 & 0.9650 & 0.0084 & 0.0070 & 0.0065 & 0.0064 & 0.0063 & 0.0062 & 146.1288 & 0.9652 & 1.8516 \\
\hline 12 & 0.9642 & 0.0092 & 0.0076 & 0.0070 & 0.0068 & 0.0067 & 0.0066 & 144.9593 & 0.9644 & 1.9954 \\
\hline 13 & 0.9640 & 0.0084 & 0.0070 & 0.0065 & 0.0064 & 0.0063 & 0.0062 & 146.1288 & 0.9642 & 1.8534 \\
\hline
\end{tabular}

\begin{tabular}{|c|c|c|c|}
\hline $\mathrm{Pg}(\mathrm{Kw})$ & Qg(Kvar) & $\mathrm{PI}(\mathrm{Kw})$ & Ql(Kvar) \\
\hline 1115 & 4430 & 1071.089195 & 4406.336789 \\
\hline \multicolumn{4}{|c|}{ Ploss (Kw) } \\
\hline \multicolumn{2}{|c|}{ Ploss_fundamental } & 35.06 & \multirow{2}{*}{43.91} \\
\hline \multicolumn{2}{|c|}{ Ploss_harmonics } & 8.85 & \\
\hline \multicolumn{4}{|c|}{ Qloss (Kvar) } \\
\hline \multicolumn{2}{|c|}{ Qloss_fundamental } & 11.78 & \multirow{2}{*}{23.66} \\
\hline \multicolumn{2}{|c|}{ Qloss harmonics } & 9.80 & \\
\hline
\end{tabular}




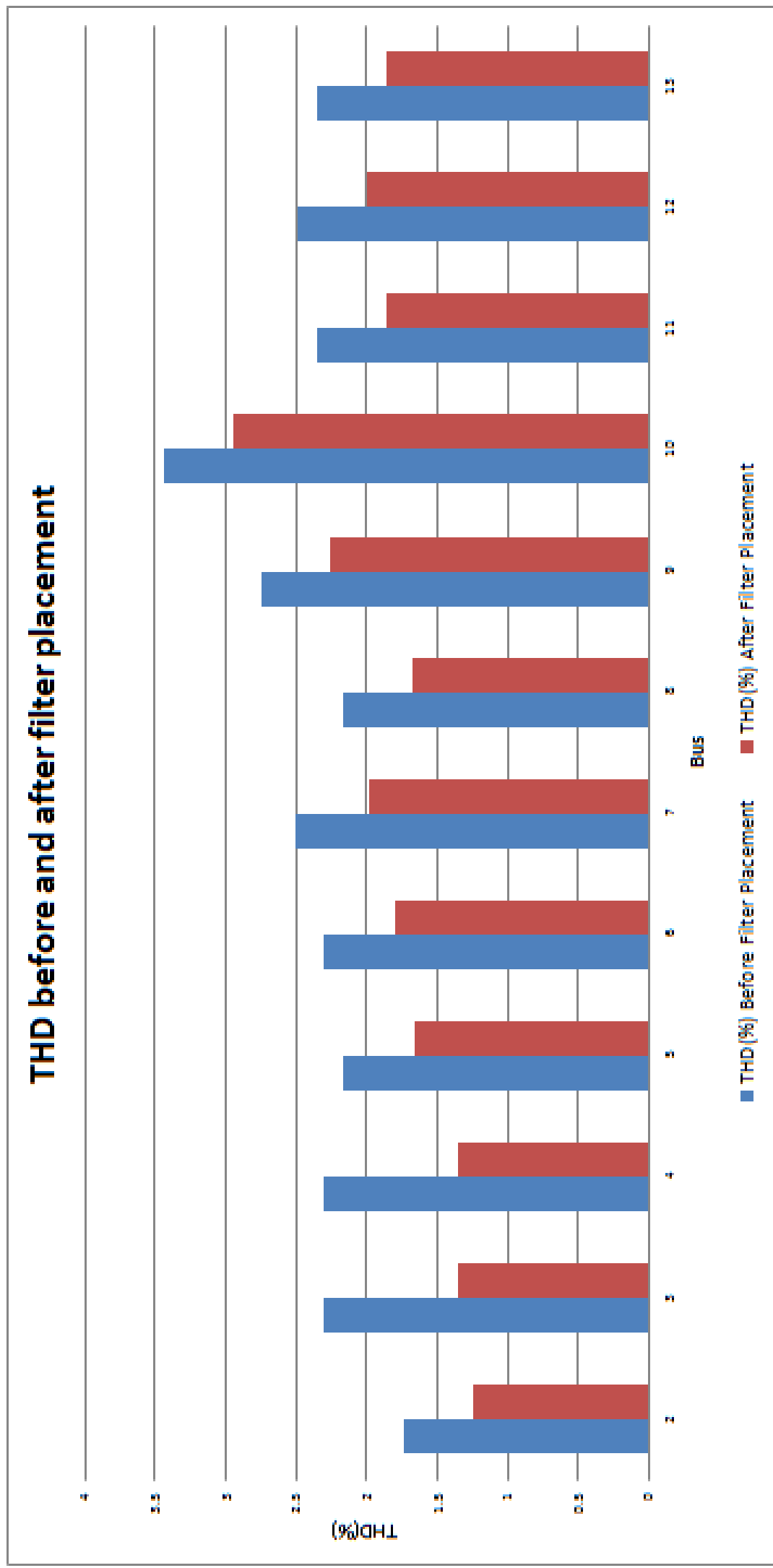

है 


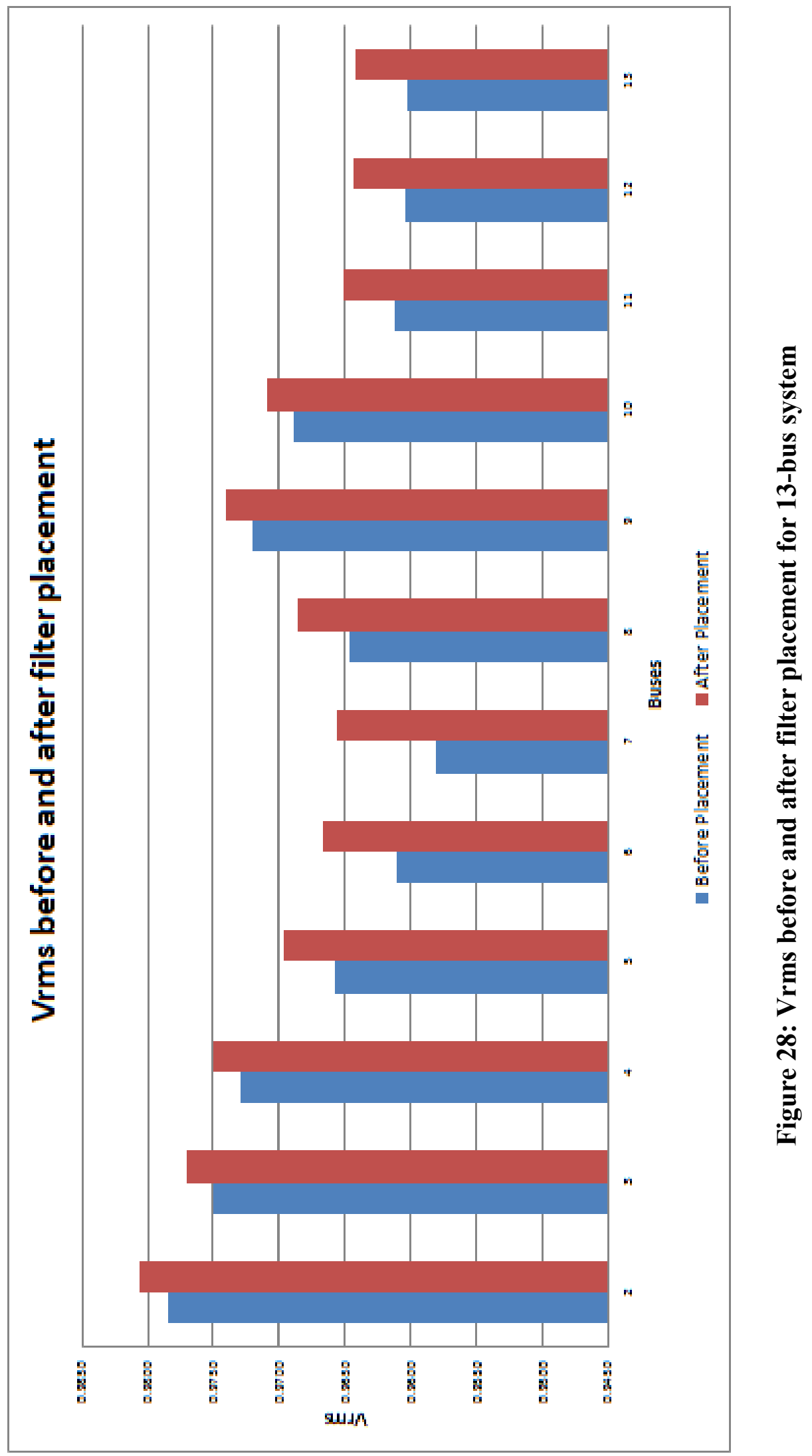


It can be seen that after placing the filter, the THD is suppressed as demonstrated in Figure 27. This has resulted in an improved voltage profile as presented in Figure 28. The following table and graph demonstrate the effect of placing filter on power losses at fundamental frequency and harmonic of each order:

Table 17: Harmonic load flow results for 13-bus after filter placement

\begin{tabular}{|l|c|c|c|}
\cline { 2 - 3 } \multicolumn{1}{c|}{} & $\begin{array}{c}\text { Before Filter } \\
\text { Placement }\end{array}$ & $\begin{array}{c}\text { After Filter } \\
\text { Placement }\end{array}$ & $\begin{array}{c}\% \\
\text { improvement }\end{array}$ \\
\cline { 2 - 3 } & Ploss(Kw) & Ploss(Kw) & \\
\hline Fundamental & 49.365 & 35.060 & $28.98 \%$ \\
\hline 3rd harmonic & 6.931 & 4.857 & $29.93 \%$ \\
\hline 5th harmonic & 2.495 & 1.748 & $29.93 \%$ \\
\hline 7th harmonic & 1.273 & 0.892 & $29.93 \%$ \\
\hline 9th harmonic & 0.770 & 0.540 & $29.93 \%$ \\
\hline 11th harmonic & 0.516 & 0.361 & $29.93 \%$ \\
\hline 13th harmonic & 0.369 & 0.259 & $29.93 \%$ \\
\hline 15th harmonic & 0.277 & 0.194 & $29.93 \%$ \\
\hline Total & $\mathbf{6 1 . 9 9 7}$ & $\mathbf{4 3 . 9 1}$ & $\mathbf{2 9 . 1 7 \%}$ \\
\hline
\end{tabular}

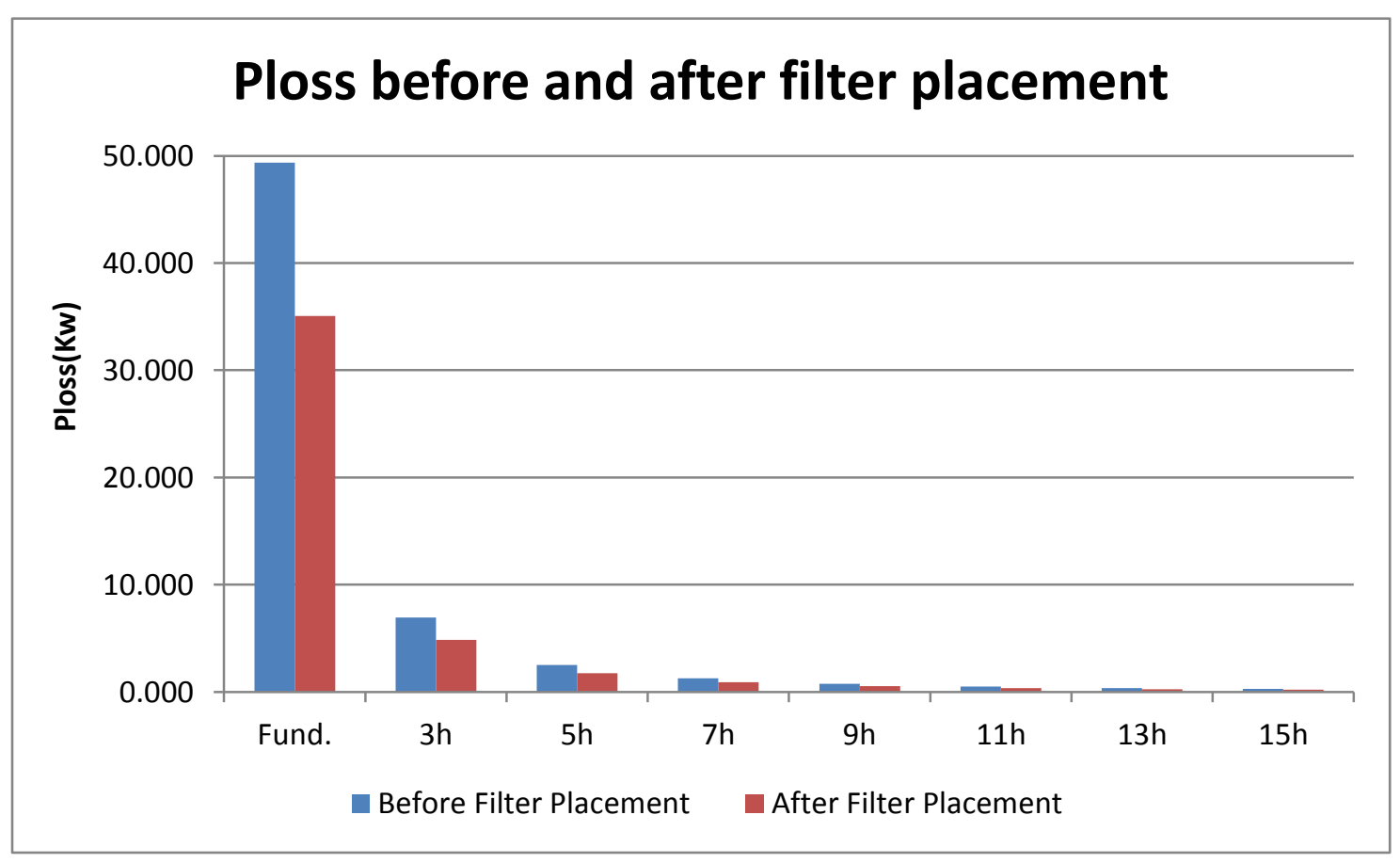

Figure 29: Ploss before and after filter placement for 13-bus system 
As a result of placing the selected filter at an optimal location, the power loss at fundamental frequency which was $49.365 \mathrm{KW}$ is improved by $28.98 \%$ to $35.060 \mathrm{KW}$. Other noticeable losses are $6.931,2.495$ and $1.273 \mathrm{KW}$ each at $3^{\text {rd }}, 5^{\text {th }}$ and $7^{\text {th }}$ order harmonics which are reduced to $4.857,1.743$ and $0.892 \mathrm{KW}$ each. $30 \%$ improvement is seen for harmonic losses. Finally, the total power loss has been reduced from $61.997 \mathrm{KW}$ to $43.91 \mathrm{KW}$ giving an overall improvement of $29.17 \%$ and a saving of almost $18 \mathrm{KW}$. 


\section{Chapter 6: Conclusion}

This thesis presents an application of the Ant Colony algorithm for optimizing filter placement and sizing on a radial distribution system in order to reduce power losses and keep the root mean squared voltages and total harmonic distortion (THD) to lie within prescribed limits. A radial distribution power flow (RDPF) and harmonic power flow (HPF) algorithms are employed which use backward-forward sweep techniques to produce power flow solution. RDPF and HPF are performed to demonstrate the effect of harmonic sources on total power loss. Then the Ant colony algorithm is used in conjunction with both power flow algorithms to select a filter size from a number of available sizes and place it at the best possible location so that both power loss and THD are minimized. Three test systems are used to validate the results.

It is seen that for 5-bus system ACS converges in 4 iterations, whereas for 11-bus it takes 18 iterations to converge and for 13-buses it takes 35 iterations to find the best possible solution. By placing the harmonic filters the effect of harmonics is suppressed. It resulted in a decrease in total real power loss and improving voltage profile. Filters are a cheaper solution and low voltage systems like the three test systems can prove to be more economically efficient solution than by using shunt capacitor as done in $[4,5,6,11$ and 19]. Furthermore ACS has proven to be fast in convergence. Best filter size chosen by ACS for all 3 test systems is $600 \mathrm{Kvar}$ the best locations found to place this filter for 5bus, 11-bus and 13-bus systems are bus 3,10 and 7 respectively and the real power losses are reduced by $86.03 \%, 31.12 \%$ and $29.17 \%$ of the power loss before placing the filter. Also, THD on every bus for all 3 test systems is also suppressed and voltage profile improved. 


\subsection{Contribution and Future Work}

It has been shown that ACS can be used to solve optimal filter placement and sizing problems. A hybrid system of RDS harmonic load flow through backward/forward sweep and ACS has been introduced. Previously this hybrid was used in [20 and 46] to solve the capacitor placement and sizing problem in RDS. In this thesis, filter is being replaced instead. Furthermore, 3 test systems are used to validate results. For different test systems ACS converges on different number of iterations.

ACS could be replaced by other heuristic methods like neural networks, PSO or honey bee mating in order to find the best solution which can converge faster. Large sized filters cost more so an economic analysis could be made by including economic factors in objective function so as to minimize the total cost of placing the best filter possible. 


\section{References}

[1] Singh, Bhim, Al-Haddad, K. ; Chandra, A, "A review of active filters for power quality improvement", Industrial Electronics, IEEE Transactions on , Volume:46, Issue: 5, pp. 960-971, 06 August 2002.

[2] IEEE Std 519-1992, IEEE Recommended Practices and Requirements for Harmonic Control in Electrical) Power Systems, New York, NY: IEEE, 1993.

[3] Yong-hua Song; Irving, M.R., "Optimisation techniques for electrical power systems. II. Heuristic optimisation methods," Power Engineering Journal, vol.15, no.3, pp.151-160, June 2001.

[4] Reza Sirjani and Badiossadat Hassanpour, "A New Ant Colony-Based Method for Optimal Capacitor Placement and Sizing in Distribution Systems", Research J. of Appl. Sci., Eng. and Technol., vol. 4, no. 8, pp. 888-891, 2012.

[5] Kyriakides and M. Polycarpou, "Short Term Electric Load Forecasting: A Tutorial", Studies in Computational Intelligence, vol. 35, pp. 391-418, SpringerVerlag Berlin Heidelberg 2007.

[6] S. Bouri, A. Zeblah, A. Ghoraf, S. Hadjeri and H. Hamdaoui, "Ant Colony Optimization to Shunt Capacitor Allocation in Radial Distribution Systems" Acta Electrotechnica et Informatica, vol. 5, no. 4, pp. 1-10, 2005.

[7] Annaluru, R.; Das, S.; Pahwa, A., "Multi-level ant colony algorithm for optimal placement of capacitors in distribution systems," Evolutionary Computation, 2004. CEC2004. Congress on, vol.2, no., pp.1932,1937 Vol.2, 19-23 June 2004.

[8] Ghorbani, M.A.; Hosseinian, S.H.; Vahidi, B., "Application of Ant Colony System algorithm to distribution networks reconfiguration for loss reduction," Optimization of Electrical and Electronic Equipment, 2008. OPTIM 2008. 11th International Conference on, vol., no., pp.269,273, 22-24 May 2008.

[9] Shyh-Jier Huang, "Enhancement of hydroelectric generation scheduling using ant colony system based optimization approaches,"IEEE Trans. Energy Conversion, vol. 16, no. 3, pp.296-301, Sept., 2001.

[10] Vlachogiannis, J.G.; Hatziargyriou, N.D.; Lee, K.Y., "Ant Colony SystemBased Algorithm for Constrained Load Flow Problem," Power Systems, IEEE Transactions on, vol.20, no.3, pp.1241,1249, Aug. 2005.

[11] Keun Yu, C.S. Chou and Y.H. Song, "Application of the Ant Colony search algorithm to short term generation scheduling problem of thermal units" Power system technology conference, Bejiin, pp. 552-556, 1998. 
[12] J. Nikoukar and M. Gandomkar. 2005. Capacitor placement in distribution networks using ant colony algorithm. In Proceedings of the 4th WSEAS International Conference on Software Engineering, Parallel $\mid \&$ Distributed Systems (SEPADS'05), Walter Dosch, Rudolf Freund, and Nikos Mastorakis (Eds.). World Scientific and Engineering Academy and Society (WSEAS), Stevens Point, Wisconsin, USA, Article 12, 5 pages.

[13] J.M. Alvarado, E.V. Alvarado, M.A. Arevalo, S.P. Quituisaca, J.F. Gomez and P.M. De Olivera-De Jesus, "Ant Colony Systems Application for Electrical Distribution Network Planning”, Intelligent system applications to power systems, Curitiba, pp. 1-6, 2009.

[14] Ziari, I.; Jalilian, A., "Optimal harmonic power flow using an ant colony system-based algorithm," Universities Power Engineering Conference, 2008. UPEC 2008. 43rd International, vol., no., pp.1,4, 1-4 Sept. 2008.

[15] A. Afshar, O. Bozorg Haddad, M.A. Mariño, B.J. Adams, "Honey-bee mating optimization (HBMO) algorithm for optimal reservoir operation", J. of the Franklin Inst., vol. 344, no. 5, pp.452-462, Aug. 2007.

[16] Omid Bozorg Haddad, Abbas Afshar, Miguel A. Mariño, "Honey-Bees Mating Optimization (HBMO) Algorithm A New Heuristic Approach for Water Resources", Springer 2006.

[17] Eberhart, R. and Kennedy, J., "A new optimizer using particle swarm theory," Proceedings of the Sixth International Symposium on Micro Machine and Human Science, pp. 39-43, 1995.

[18] Hu, X., Shi, Y. and Eberhart, R., "Recent advances in particle swarm, "Proceedings of 2004 Congress on Evolutionary Computation, vol.1, pp. 90-97, 2004.

[19] Arefi, A.; Haghifam, M.R.; Fathi, S.H., "Distribution harmonic state estimation based on a modified PSO considering parameters uncertainty," PowerTech, 2011 IEEE Trondheim, vol., no., pp.1,7, 19-23 June 2011.

[20] Eajal, A.A.; El-Hawary, M.E., "Optimal Capacitor Placement and Sizing in Unbalanced Distribution Systems With Harmonics Consideration Using Particle Swarm Optimization," Power Delivery, IEEE Transactions on , vol.25, no.3, pp.1734,1741, July 2010.

[21] Hamed Aly, "Forecasting, Modeling and Control of Tidal Currents Electrical Energy Systems" Ph.D dissertation, Dept. Elect. Eng., Dalhousie Univ., Halifax, N.S., 2012.

[22] Barros, J.; Diego, R.I.; De Apraiz, M., "Applications of wavelet transforms in electric power quality: Harmonic distortion," Applied Measurements for Power 
Systems (AMPS), 2011 IEEE International Workshop on , vol., no., pp.13,18, 28-30 Sept. 2011

[23] A. W. Galli, G. T. Heydt and P.F. Ribeiro, "Exploring the Power of Wavelet Analysis" IEEE Computer Application in Power pp. 37-41, 1999.

[24] P.F. Ribeiro, "Wavelet transform: an advanced tool for analyzing non-stationary harmonic distortion in power systems," IEEE International Conference on Harmonics in Power Systems 1994, ICHPS VI, Bologna, Italy, September 2123, 1994, pp. 365-369.

[25] S. Santoso, E.J. Powers, and W.M. Grady, "Electric power quality disturbance detection using wavelet transform analysis," IEEE-SP International Symposium on Time-Frequency and Time Scale Analysis, Philadelphia, USA, 1994, pp. 166-169.

[26] D. Robertson, O.I. Camps, and J. Mayer, "Wavelets and power system transients: feature detection and classification," International Symposium on Optical Engineering in Aerospace Sensing, SPIE vol. 2242, 1994, pp. 474-487.

[27] Gonzalo Sandoval, John Houdek, "A Review of Harmonic Mitigation Techniques, on line April 15, 2014 www.artechepq.com/assets/files/aReviewofharmMitigTech.pdf._(accessed on April 14, 2014)

[28] Sekar, T.C.; Rabi, B.J., "A review and study of harmonic mitigation techniques," Emerging Trends in Electrical Engineering and Energy Management (ICETEEEM), 2012 International Conference on, vol., no., pp.93,97, 13-15 Dec. 2012.

[29] Myron Zuker, "Line Reactor Manual”, URL: http://www.myronzucker.com/Resources/Application-Guides/LineReactor.html. (accessed on April 14, 2014)

[30] Care Base, "Isolation Transformer Line Conditioner", URL: http://www.carebase.com/Isolation-transformer-line-conditioner-neutralground-bond-EI-core-low-impedance-120V-120vac-600VA-1000VA-1kVAILc-600B-1000B.htm. (accessed on April 14, 2014)

[31] Louis Hapeshis P.E., "Why Worry About Harmonics?”URL: www.flowcontrolnetwork.com/articles/why-worry-about-harmonics(accessed on April 14, 2014)

[32] Mau Teng Au, Jovica V. Milanovic, "Planning approaches for the strategic placement of passive harmonic filters in radial distribution networks". IEEE Trans. Power Del., vol. 22, no. 1, pp. 347-353, Jan., 2007. 
[33] Chang, G.W.; Shou-Yung Chu; Hung-Lu Wang, "Sensitivity-based approach for passive harmonic filter planning in a power system," Power Engineering Society Winter Meeting, 2002. IEEE, vol.2, no., pp.937,940 vol.2, 2002.

[34] Chang, G.W.; Chu, S.Y.; Wang, H.L., "A new approach for placement of single-tuned passive harmonic filters in a power system," Power Engineering Society Summer Meeting, 2002 IEEE, vol.2, no., pp.814,817 vol.2, 25-25 July 2002.

[35] Chi-Jui Wu; Jung-Chen Chiang; Shih-Shong Yen; Ching-Jung Liao; Jin-Shyr Yang; Tzong-Yih Guo, "Investigation and mitigation of harmonic amplification problems caused by single-tuned filters," Power Delivery, IEEE Transactions on , vol.13, no.3, pp.800,806, July 1998.

[36] Naimish Zaveri, Ajitsinh Chudasama, "Control strategies for harmonic mitigation and power factor correction using shunt active filter under various source voltage conditions", International Journal of Electrical Power \& Energy Systems, Volume 42, Issue 1, November 2012, Pages 661-671, ISSN 01420615.

[37] Terciyanli, A.; Avci, T.; Yilmaz, I.; Ermis, C.; Kose, N.; Acik, A.; Kalaycioglu, A.; Akkaya, Y.; Cadirci, I.; Ermis, M., "A Current Source Converter based Active Power Filter for mitigation of harmonics at the interface of distribution and transmission systems," Energy Conversion Congress and Exposition (ECCE), 2010 IEEE, vol., no., pp.320,327, 12-16 Sept. 2010.

[38] Anup Kumar Panda, Suresh Mikkili, "FLC based shunt active filter ( $p-q$ and Id-Iq) control strategies for mitigation of harmonics with different fuzzy MFs using MATLAB and real-time digital simulator", International Journal of Electrical Power \& Energy Systems, Volume 47, May 2013, Pages 313-336, ISSN 0142-0615.

[39] Guerin, P.; Miegeville, L., "Optimal placement and sizing of harmonic filters aboard an electric propulsion ship," Generation, Transmission \& Distribution, IET, vol.1, no.4, pp.613,618, July 2007.

[40] Chang W. Gary, Hung-Lu Wang, and Shou-Yung Chu, "Strategic placement and sizing of passive filters in a power system for controlling voltage distortion”. IEEE Transactions on Power Delivery, vol. 19, no.3, pp. 12041211, 2004.

[41] Indranil Bhattacharya, Yuhang Deng and Simon Y. Foo, "Active filters for harmonics elimination in solar photovoltaic grid connection and standalone systems", 2nd Asia Symposium on Quality Electronic Design, 2002.

[42] Chih-Ju Chou; Chih-Wen Liu; June-Yown Lee; Kune-Da Lee, "Optimal planning of large passive-harmonic-filters set at high voltage level," Power Systems, IEEE Transactions on, vol.15, no.1, pp.433,441, Feb 2000. 
[43] Teng, J. H., "Network-topology-based three-phase load flow for distribution systems," Proc. Natl. Sci. Counc. ROC(A), vol.24, no.4, pp. 259-264, 2000.

[44] Teng, J. and Chang, C, "Backward/Forward sweep-based harmonic analysis method for distribution systems," IEEE Transactions on Power Delivery, vol.22, no.3, pp. 1665-1672, 2007.

[45] EATON CORPORATION Cutler-Hammer, Low Voltage Power Factor Correction pp. 20, "Low Voltage Switched Harmonic Filters", URL: http://www.eaton.com/ecm/idcplg?IdcService=GET FILE\&allowInterrupt $=1 \&$ RevisionSelectionMethod $=$ LatestReleased\&Rendition $=$ Primary\&dDocName $=\mathrm{T}$ D02607001E (accessed March 25, 2014).

[46] Fitriana Suhartati, Dimas Fajar U. P., Ontoseno Penangsang, and Adi Soeprijanto, "Capacitor Placement and Sizing in Distorted Distribution Systems Using Simplified Direct Search Algorithm," Journal of Clean Energy Technologies vol. 2, no. 4, pp. 317-321, 2014. 